Dois métodos para a investigação de ciclos limites que bifurcam de centros 
SERVIÇO DE PÓS-GRADUAÇÃO DO ICMC-USP

Data de Depósito: 23/03/2011

Assinatura:

\title{
Dois métodos para a investigação de ciclos limites que bifurcam de centros
}

\author{
Alex Carlucci Rezende
}

Orientadora: Profa. Dra. Regilene Delazari dos Santos Oliveira

Dissertação apresentada ao Instituto de Ciências Matemáticas e de Computação - ICMC-USP, como parte dos requisitos para obtenção do título de Mestre em Ciências - Matemática. VERSÃO REVISADA.

USP - São Carlos

Março/2011 
Ficha catalográfica elaborada pela Biblioteca Prof. Achille Bassi e Seção Técnica de Informática, ICMC/USP, com os dados fornecidos pelo(a) autor(a)

\begin{tabular}{|c|c|}
\hline \multirow[t]{3}{*}{ R467d } & $\begin{array}{l}\text { Rezende, Alex Carlucci } \\
\quad \text { Dois métodos para a investigação de ciclos limites } \\
\text { que bifurcam de centros / Alex Carlucci Rezende; } \\
\text { orientadora Regilene Delazari dos Santos Oliveira -- } \\
\text { São Carlos, } 2011 \text {. } \\
\quad 119 \text { p. }\end{array}$ \\
\hline & $\begin{array}{l}\text { Dissertação (Mestrado - Programa de Pós-Graduação en } \\
\text { Matemática)-- Instituto de Ciências Matemáticas e } \\
\text { de Computação, Universidade de São Paulo, } 2011 .\end{array}$ \\
\hline & $\begin{array}{l}\text { 1. bifurcação de centros. 2. XVI problema de } \\
\text { Hilbert. 3. integral abeliana. 4. método do } \\
\text { averaging. I. Oliveira, Regilene Delazari dos } \\
\text { Santos, orient. II. Título. }\end{array}$ \\
\hline
\end{tabular}


"Há homens que lutam um dia e são bons. Há outros que lutam um ano e são melhores. Há os que lutam muitos anos e são muito bons. Porém, há os que lutam toda a vida. Esses são os imprescindíveis." 


\section{Agradecimentos}

Há momentos na vida em que todos nós ficamos atarefados e nem nos damos conta de que ao nosso lado estão pessoas que fazem tudo valer a pena! Com tanta correria no dia a dia, ficamos acomodados e esquecemo-nos de agradecer por tudo que acontece conosco: a ajuda recebida, a graça alcançada. Neste momento, paro por um instante e tento agradecer aquele sorriso, aquele puxão de orelha, aquelas palavras de conforto e também de repreensão, aquele carinho, aquela tristeza e aquela alegria. Certamente, tudo isso faz-me querer ser uma pessoa melhor a cada dia.

Em primeiro lugar, quero agradecer a Deus por minha vida, minha família e amigos, meus sentidos, minha sabedoria e pela dose diária de vontade e ânimo.

Agradeço e dedico este trabalho a minha mãe, Lucimary, a quem eu devo toda minha essência como pessoa e cidadão. Te amo para sempre, mãe!

Agradeço também ao meu pai, Wagner, que pôde proporcionar-me a oportunidade de ter uma boa formação escolar básica; além de ensinar-me como (não) agir em determinadas situações.

Ao meu irmão, irmãs, sobrinho, padrasto, cunhado, cunhada, avó, tios, tias, primos e primas, agradeço pelos momentos felizes em família, pois toda vez que estive em Presidente Prudente era motivo de festas, churrascos e muito carinho. Agradeço também aos meus tios Ademar e Lourdes Pelosi, e também ao Murillo, Daviani, Gislaine e Marcos, pela companhia agradável em São Carlos, pela confiança e pela ótima recepção desde 2008.

Agradeço a todos os professores e funcionários do ICMC/USP, em especial a minha orientadora Regilene Oliveira que sempre esteve pronta para sanar minhas dúvidas, com 
muita paciência e dedicação. Com certeza, Regilene, a senhora é em quem me espelho quanto profissional: uma pessoa competente, inteligente e atenciosa! E também agradeço ao professor Nivaldo Grulha pela amizade e pela ajuda. Por fim, e não menos importante, agradeço aos professores Cláudio A. Buzzi (IBILCE/Unesp) e Joan C. Artés (UAB/Barcelona) pela ajuda durante o desenvolvimento deste trabalho. Muito obrigado!

Agradeço também aos professores da FCT/Unesp de Presidente Prudente, em especial ao Marcelo Messias e a Monica Fürkotter. Obrigado Marcelo por ter confiado no meu trabalho e dado a oportunidade para que eu iniciasse a pesquisa científica. E Monica, obrigado por todos os conselhos e dicas; como já disse, a senhora tem uma parcela de culpa por eu estar onde estou hoje. Serei eternamente grato!

Aos responsáveis por minhas grandes amizades: Thaís Dalbelo, Monica Faustino, Jéssica Fernandes, Lidiane Hieda e Flávio Pires pelos momentos bons e ruins durante a graduação e a vida. Aos amigos que fiz há pouco, mas que já fazem parte de minha história: Rafael Figueiredo, Matheus Guerrero, Rodrigo Brum, José Augusto Stuchi, amigos de república e companheiros para vida inteira; Juliana Theodoro de Lima, Matheus Bortolan, Andrea Sacramento, Ingrid Sarmiento, Jackson Itikawa, Andreza Beezão, Apoenã Passamani, Ana Carolina Ramos, Henry Mercado, Nelson Silva, Northon Penteado, Rafael Gonzalez, Vinicius Laass e demais colegas de turma de mestrado e doutorado, pelos momentos bons e ruins que tivemos no ICMC e em São Carlos, pelos cafés, passeios, reuniões de estudo, troca de listas de exercícios e deliciosos churrascos e almoços. E também agradeço ao meu amigo Rodrigo Euzébio, pela ajuda com alguns tópicos deste trabalho e pelos conselhos, risadas e amizade. Valeu por tudo!

Agradeço à Coordenação de Aperfeiçoamento de Pessoal de Nível Superior (CAPES) pelos seis primeiros meses de bolsa de Mestrado e à Fundação de Amparo à Pesquisa do Estado de São Paulo (FAPESP) pelos demais meses de suporte financeiro.

E por fim, fazendo minhas as palavras de Chico Xavier: "Deus nos concede, a cada dia, uma página de vida nova no livro do tempo. Aquilo que colocarmos nela, corre por nossa conta!" 


\section{Resumo}

Um dos mais investigados problemas na teoria qualitativa dos sistemas dinâmicos no plano é o XVI problema de Hilbert que trata dos ciclos limites. Mais precisamente, a segunda parte do referido problema questiona sobre o número máximo de ciclos limites de um sistema diferencial polinomial plano de grau $n$. Por ciclo limite entendemos uma órbita fechada isolada no conjunto de todas as órbitas periódicas de um sistema diferencial plano. Uma maneira clássica de obter um ciclo limite é perturbando um sistema com uma singularidade do tipo centro. Nesta dissertação apresentamos dois métodos utilizados para a análise do número de ciclos limites que bifurcam de um centro, a saber o método das integrais abelianas e o método do averaging.

Palavras-chave: Bifurcação de centros, XVI problema de Hilbert, integral abeliana, método do averaging. 


\section{Abstract}

One of the most investigated problems in the qualitative theory of dynamical systems in the plane is the XVI Hilbert's problem which deals with limit cycles. More precisely, the second part of the problem asks about the maximum number of limit cycles of a polynomial differential system of degree $n$. A limit cycle is a single closed orbit on the set of all periodic orbits of a differential planar system. A classic way to obtain a limit cycle is perturbing a system with a singularity of center type. In this work we discuss about two methods used to investigate the number of limit cycles which bifurcate from a center; they are known as Abelian integrals and averaging theory.

Key words: Bifurcation of centers, XVI Hilbert's problem, Abelian integral, averaging method. 


\section{Sumário}

Introdução

1 Integrais Abelianas 5

1.1 Sistemas polinomiais quadráticos no plano . . . . . . . . . . . . . . . 6

1.2 O XVI problema de Hilbert . . . . . . . . . . . . . . . . . . 8

1.2.1 Configuração de ciclos limites . . . . . . . . . . . . . . . . . 10

1.3 A versão fraca do XVI problema de Hilbert . . . . . . . . . . . . . . . . . . 10

1.4 Integrais abelianas e ciclos limites . . . . . . . . . . . . . . . . . 13

1.4.1 O teorema de Poincaré-Pontryagin . . . . . . . . . . . . . . . 13

1.5 Estimativa do número de zeros das integrais abelianas . . . . . . . . . . . 22

1.5.1 O método baseado na equação de Picard-Fuchs . . . . . . . . . . . 23

1.6 Estudo do sistema $P_{23} \quad \ldots \ldots \ldots$. . . . . . . . . . . . . . . . . . . . . . . . . . . . 29

$\begin{array}{lll}2 & \text { A teoria do averaging } & 37\end{array}$

2.1 Preliminares . . . . . . . . . . . . . . . . . . . 38

2.1.1 Teorema de existência e unicidade de soluções . . . . . . . . . . . . 38

2.1.2 Função ordem, símbolos de Landau e aproximação assintótica . . . 39

2.2 Averaging periódico de primeira ordem analítico . . . . . . . . . . . . 45

2.2.1 Primeira prova do teorema do averaging de primeira ordem . . . . . 47

2.2.2 Segunda prova do teorema do averaging de primeira ordem . . . . . 52

2.3 A forma padrão . . . . . . . . . . . . . . . . . . . . . . . . 64

2.3.1 A reformulação na forma padrão . . . . . . . . . . . . . . . . 66 
2.3.2 A forma padrão no caso quasilinear . . . . . . . . . . . . . . 68

2.3.3 A importância da forma padrão . . . . . . . . . . . . . . 75

2.4 Averaging periódico de alta ordem analítico . . . . . . . . . . . 77

2.5 Averaging periódico via grau de Brouwer . . . . . . . . . . . . . . 85

2.5.1 Teoria do grau em espaço de dimensão finita . . . . . . . . . . . . 87

2.5.2 Algumas observações sobre o grau de Brouwer . . . . . . . . . . . . 89

2.5.3 Averaging via grau de Brouwer . . . . . . . . . . . . . . . 94

2.5.4 Averaging versus integrais abelianas em sistemas integráveis . . . . 105

3 Considerações finais 


\section{Introdução}

O que podemos dizer sobre o número e a posição de ciclos limites em sistemas diferenciais no plano de grau $n$ ?

É bem conhecido que as soluções de um sistema diferencial são pontos, retas ou órbitas periódicas ([15], Teorema 1.4, p. 4). Quando uma órbita periódica for isolada no conjunto de todas as órbitas periódicas do sistema, será chamada de ciclo limite. A noção de ciclo limite surgiu pela primeira vez nos estudos das equações diferenciais no plano realizados por Poincaré entre os anos de 1880 e 1890, e desde então os ciclos limites são o objeto de estudo de muitos pesquisadores. Por exemplo, Van der Pol em 1926, Liénard em 1928 e Andronov em 1929 provaram que a solução periódica de uma oscilação autossustentada de um circuito em um tubo de vácuo era um ciclo limite, no sentido definido por Poincaré. Observada a presença de um ciclo limite na natureza, pesquisadores de diversas áreas da ciência passaram a observar a existência ou não e, ao mesmo tempo, o número máximo de ciclos limites, bem como outras propriedades.

Entretanto, para os matemáticos, este problema já os instigava desde o início do século. Em 1900, durante a Conferência Internacional de Matemáticos de Paris, Hilbert [24, 25] propôs uma lista com vinte e três problemas matemáticos para serem resolvidos no século XX. Dentre eles, destacamos o décimo sexto problema por se tratar exatamente de ciclos limites em sistemas diferenciais no plano.

Certamente, o XVI problema de Hilbert é um dos mais investigados problemas na teoria qualitativa dos sistemas dinâmicos no plano. Originalmente, Hilbert formulou seu XVI problema dividindo-o em duas partes. A primeira delas é de interesse da geometria 
algébrica, e a segunda questiona sobre o número máximo e a posição relativa de ciclos limites de sistemas polinomiais no plano

$$
\left\{\begin{array}{l}
\dot{x}=P(x, y), \\
\dot{y}=Q(x, y),
\end{array}\right.
$$

onde $P$ e $Q$ são polinômios nas variáveis $x$ e $y$ e o máximo entre os graus de $P$ e $Q$ é $n$. Segundo Li em [29], Hilbert apontou possíveis conexões entre as duas partes do problema, mas que não é o foco desta dissertação.

Apesar do grande número de trabalhos nessa área, o progresso alcançado na busca pela resposta a este problema não foi grande. Por exemplo, embora Écalle em [16] e Il'Yashenko em [27] terem provado que o número de ciclos limites em tais sistemas é finito, suas demonstrações não são muito acessíveis. Além disso, nem mesmo foi provada a existência de uma cota superior uniforme para o número de ciclos limites de sistemas quadráticos. Suspeita-se que esta cota uniforme exista e seja 4, e que a única configuração de ciclos limites de campos quadráticos com 4 ciclos limites é a $(3,1)$, isto é, três ciclos limites ao redor de um foco e um ciclo limite ao redor de outro foco (mais detalhes em [46]).

Em vista da dificuldade de resolução do XVI problema de Hilbert como fora proposto, vários estudiosos foram aprimorando e dando novos enunciados ao problema. Por exemplo, Arnol'd $[2,4]$ propôs a investigação do número máximo de ciclos limites que bifurcam de uma singularidade do tipo centro, conhecida como a versão fraca do XVI problema de Hilbert.

Segundo Li, Li, Llibre e Zhang [30], existem diversos métodos para estudar a bifurcação de ciclos limites que bifurcam de um centro. A maioria deles são baseados na aplicação de primeiro retorno de Poincaré, como é o caso do método da integral de Poincaré-Melnikov e do método das integrais abelianas. Recentemente, alguns outros métodos foram apresentados, uns baseados no fator de integração inverso e outros, na redução do problema a uma equação diferencial em uma variável, no caso do método do averaging, por exemplo.

Para o plano, os métodos da integral de Poincaré-Melnikov e das integrais abelianas são equivalentes (veja [7]). Vale observar que os dois primeiros métodos fornecem as órbitas 
de sistemas não perturbados que se tornam ciclos limites após perturbações. O método das integrais abelianas se aplica apenas para dimensão dois. O terceiro e quarto métodos fornecem o número e forma dos ciclos limites dos sistemas obtidos por perturbações de qualquer ordem em todas as dimensões.

Esta dissertação propõe-se a apresentar de maneira criteriosa as duas técnicas mais conhecidas e utilizadas no estudo de ciclos limites que bifurcam de um centro em sistemas planos, conhecidas como integrais abelianas e a teoria do averaging. Dessa maneira, a dissertação tem duas partes distintas, cada uma delas destinada ao estudo de uma das técnicas mencionadas.

Subdividimos o estudo das integrais abelianas em seções. Na primeira seção apresentamos uma introdução ao problema e definições básicas ao estudo. Em seguida, tratamos do Teorema de Poincaré-Pontryagin e, finalmente, descrevemos um dos métodos utilizados para a estimativa do número de zeros das integrais abelianas. Para concluir a primeira parte desta dissertação, investigamos um sistema quadrático com integral primeira racional de grau três e descrevemos as dificuldades encontradas durante o estudo.

A segunda parte desta dissertação, onde tratamos da teoria do averaging também foi dividida em seções. Na primeira delas apresentamos as definições e ferramentas básicas ao estudo. Em seguida, tratamos do averaging de primeira ordem e de ordem mais alta e descrevemos o que é conhecido como forma padrão do sistema. Finalmente, tratamos do averaging periódico via grau de Brouwer. Em todas as seções exemplos são apresentados visando facilitar a compreensão das técnicas discutidas.

Encerramos a dissertação com algumas considerações finais e com as referências bibliográficas utilizadas para a preparação da mesma.

Vale ressaltar que utilizaremos as palavras averaging, derivada do inglês, e promediado, derivada do castelhano, assim como estão grafadas. Isso se deve ao fato da impossibilidade de uma boa tradução e, também, pelo uso corriqueiro na literatura e por pesquisadores da área.

Gostaríamos de agradecer aos professores membros da banca, Marco Antonio Teixeira (IME/Unicamp) e Luis Fernando de Osório Mello (UNIFEI), pelas sugestões e correções 
que muito contribuíram para a conclusão desta dissertação, tornando-a um texto mais didático e compreensível. 


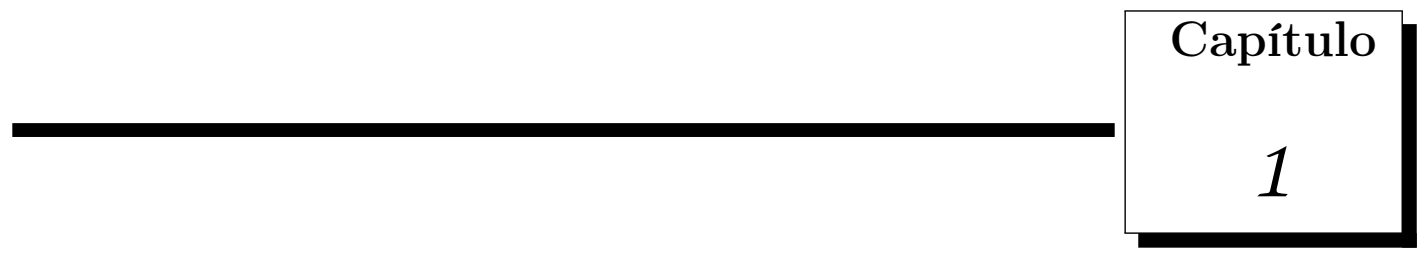

\section{Integrais Abelianas}

Nosso objetivo neste capítulo é apresentar o método conhecido como integral abeliana, utilizado para estimar o número de ciclos limites que bifurcam de um centro, no estudo da versão fraca do XVI problema de Hilbert.

Iniciamos o capítulo com definições e resultados básicos e fundamentais para a compreensão da técnica a ser apresentada. Em seguida, apresentamos o Teorema de PoincaréPontryagin, que exibe a relação entre o número de zeros de uma integral abeliana e o número máximo de ciclos limites que bifurcam de um centro por uma perturbação, seguido de um exemplo e comentários para casos mais gerais.

Na seção seguinte, discutimos a respeito de uma técnica associada às integrais abelianas, o método envolvendo da equação de Picard-Fuchs. Essa técnica é uma ferramenta auxiliar na estimativa do número de zeros da integral abeliana e, consequentemente, do número de ciclos limites do sistema perturbado.

Finalizamos o capítulo discutindo sobre o estudo do número de ciclos limites que bifurcam do centro de um sistema quadrático fixado. Nesse exemplo, as técnicas descritas na seção anterior foram aplicadas. Discutimos resultados e apresentamos detalhes e dificuldades encontradas. 


\subsection{Sistemas polinomiais quadráticos no plano}

Seja $\mathbb{R}[x, y]$ o anel de polinômios nas variáveis $x$ e $y$ com coeficientes em $\mathbb{R}$. Consideremos um sistema de equações diferenciais polinomiais ou simplesmente um sistema diferencial em $\mathbb{R}^{2}$ definido por

$$
\left\{\begin{array}{l}
\dot{x}=P(x, y), \\
\dot{y}=Q(x, y),
\end{array}\right.
$$

onde $P, Q \in \mathbb{R}[x, y]$. Dizemos que o máximo dos graus dos polinômios $P$ e $Q$ é o grau do sistema (1.1.1). Um sistema diferencial quadrático ou simplesmente um sistema quadrático é um sistema diferencial polinomial de grau 2. Diremos que o sistema quadrático (1.1.1) é não degenerado se os polinômios $P$ e $Q$ forem relativamente primos, ou coprimos.

Seja $\mathcal{U}$ um subconjunto aberto e denso de $\mathbb{R}^{2}$. Diremos que uma função não constante

$$
H: \mathcal{U} \rightarrow \mathbb{R}
$$

é a integral primeira do sistema (1.1.1) em $\mathcal{U}$ se $H(x(t), y(t))$ for constante para todos os valores de $t$ para os quais $(x(t), y(t))$ é uma solução do sistema (1.1.1).

Proposição 1.1.1. $H: \mathcal{U} \rightarrow \mathbb{R}$ será integral primeira do sistema (1.1.1) se, e somente se,

$$
P(x, y) \frac{\partial H}{\partial x}(x, y)+Q(x, y) \frac{\partial H}{\partial y}(x, y)=0
$$

para todo $(x, y) \in \mathcal{U}$.

Demonstração: Seja $(x(t), y(t)) \in \mathcal{U}$ uma solução de (1.1.1). $H$ será integral primeira de (1.1.1) se, e somente se, $H(x(t), y(t))=h$, para todo $t$ tal que $(x(t), y(t))$ é solução de (1.1.1) em $\mathcal{U}$.

Logo,

$$
\frac{\partial}{\partial t} H(x(t), y(t))=0 \Leftrightarrow \frac{\partial H}{\partial x}(x, y) \cdot \dot{x}+\frac{\partial H}{\partial y}(x, y) \cdot \dot{y}=0
$$

O sistema diferencial (1.1.1) é dito integrável se existir $H$ integral primeira do sistema. 
Dada $f \in \mathbb{R}[x, y]$, diremos que a curva $f(x, y)=0$ é uma curva algébrica invariante do sistema (1.1.1) se existir $K \in \mathbb{R}[x, y]$ tal que

$$
P \frac{\partial f}{\partial x}+Q \frac{\partial f}{\partial y}=K f
$$

O nome "invariante" para a curva algébrica $f(x, y)=0$ deve-se ao fato que, se uma trajetória intercepta a curva $f(x, y)=0$, então toda trajetória está contida nela.

A busca por integral primeira para um sistema diferencial planar é uma ferramenta clássica para descrever o retrato de fase do mesmo. Como usual, o retrato de fase de um sistema diferencial é a decomposição do domínio de definição do sistema como união de todas suas órbitas, ou trajetórias.

Se a integral primeira $H$ for uma função racional, então diremos que $H$ é uma integral primeira racional. Para uma integral primeira racional sempre assumimos que os polinômios do numerador e do denominador sejam coprimos. Se o máximo entre os graus dos polinômios do numerador e denominador de uma integral primeira racional for $m$, então diremos que a integral primeira racional $H$ tem grau $m$. É claro que um caso particular de integrais primeira racionais são as integrais primeira polinomiais, isto é, quando o denominador da integral primeira racional for uma constante não nula.

Notemos que, se um sistema diferencial polinomial tiver uma integral primeira racional, então todas as trajetórias desse sistema diferencial estarão contidas em curvas algébricas invariantes.

Os sistemas de equações diferenciais em $\mathbb{R}^{2 n}$ da forma

$$
\left(\begin{array}{c}
\dot{q}_{i} \\
\dot{p}_{i}
\end{array}\right)=\left(\begin{array}{c}
-\frac{\partial H}{\partial p_{i}} \\
\frac{\partial H}{\partial q_{i}}
\end{array}\right) \text {, }
$$

onde $\left(q_{1}, \ldots, q_{n}, p_{1}, \ldots, p_{n}\right)$ são as coordenadas em $\mathbb{R}^{2 n}$ e $H: \mathbb{R}^{2 n} \rightarrow \mathbb{R}$ é uma função, serão chamados de sistemas hamiltonianos com $n$ graus de liberdade e $H$ será chamada função hamiltoniana do sistema.

Uma das técnicas utilizadas na busca por soluções de equações diferenciais é o método do fator integrante. Uma função $M=M(x, y)$ é um fator de integrante, ou fator de 
integração, do sistema (1.1.1) se uma das seguintes condições equivalentes for satisfeita:

1. $\frac{\partial(M P)}{\partial x}=-\frac{\partial(M Q)}{\partial y}$;

2. $\operatorname{div}(M P, M Q)=0$.

Observação 1.1.2. A integral primeira $H$ associada ao fator de integração $M$ é dada de forma que

$$
\dot{x}=M P=\frac{\partial H}{\partial y} \text { e } \dot{y}=M Q=-\frac{\partial H}{\partial x} .
$$

Reciprocamente, dada uma integral primeira $H$ do sistema (1.1.1) sempre podemos encontrar um fator de integração $M$ para o qual (1.1.4) fique satisfeita.

\subsection{O XVI problema de Hilbert}

Os problemas de Hilbert $[24,25]$ formam um rol de vinte e três problemas em Matemática que foram propostos pelo matemático alemão David Hilbert (1862-1943) durante o Congresso Internacional de Matemáticos de Paris em 1900. Eles influenciaram a Matemática do século XX. De acordo com Corry [14], "esse conjunto de problemas tornou-se um verdadeiro objeto de culto, de maneira que, ao passar dos anos, vários matemáticos dedicaram seus esforços em resolvê-los, e os que tiveram êxito cobriram-se de glória profissional", como Kurt Gödel (1906-1978), Max Dehn (1878-1952), Andrei Nikolaevich Kolmogorov (1903-1987) e Vladimir Igorevich Arnol'd (1937-2010).

O XVI problema de Hilbert é um dos poucos problemas que permanecem em aberto. Ele trata dos ciclos limites em sistemas diferenciais polinomiais e pode ser dividido em duas partes.

A segunda parte do referido problema (a primeira parte lida com ovais no conjunto de zeros de funções algébricas) pode ser enunciado da seguinte maneira: Qual é o número máximo (chamado de número de Hilbert e denotado por $H(n)$ ) de ciclos limites de (1.1.1) para todos os $P$ e $Q$ possiveis? E o que dizer das posições relativas dos ciclos limites de $(1.1 .1)$ ? 
Definição 1.2.1. [15] Uma órbita periódica $\gamma$ do sistema (1.1.1) será chamada de ciclo limite se existir uma vizinhança $V$ de $\gamma$ tal que $\gamma$ seja a única órbita periódica contida em $V$.

Em muitas aplicações o número e as posições de ciclos limites são importantes para entender o comportamento dinâmico do sistema.

Notemos que o problema é trivial para $n=1$. Um sistema linear pode ter órbitas periódicas, mas não possui ciclos limites. Por exemplo, consideremos o sistema

$$
\left\{\begin{array}{l}
\dot{x}=y, \\
\dot{y}=-x
\end{array}\right.
$$

cujas órbitas são circunferências concêntricas na origem. Observe que não há nenhum ciclo limite em seu retrato de fase, como mostra a Figura 1.1, pois todas as suas órbitas, exceto a de equilíbrio, são fechadas. Dessa forma, assumimos $n \geq 2$.

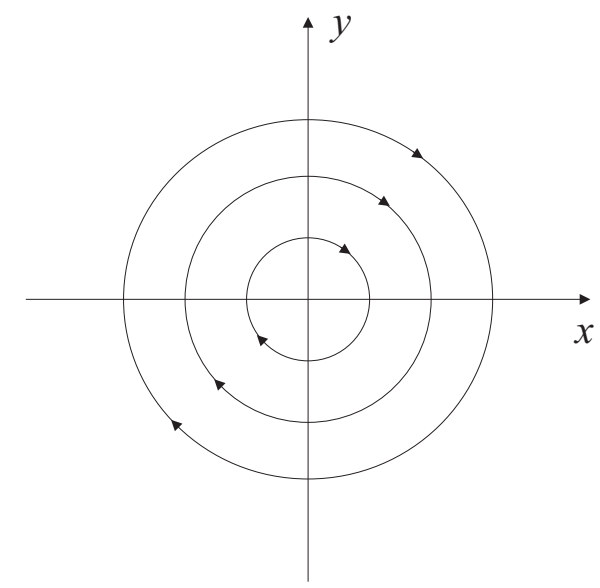

Figura 1.1: Retrato de fase do sistema (1.2.1).

Em geral, sabe-se muito pouco sobre o número de ciclos limites de (1.1.1), mesmo para o caso $n=2$. Em contrapartida, podemos afirmar que um sistema desse tipo possui um número finito de ciclos limites. Existem duas demonstrações (independentes) para esse fato que são devidas a Écalle [16] e a Il'Yashenko [27] e vale observar que ambas não foram ainda completamente compreendidas pela comunidade científica. Não se sabe, entretanto, nem mesmo para o caso quadrático, se existe um limitante superior uniforme, dependo do grau do sistema. 


\subsubsection{Configuração de ciclos limites}

Além de questionar a existência de um limitante superior uniforme, a segunda parte do XVI problema de Hilbert também questiona sobre uma descrição das possíveis configurações de ciclos limites que sistemas polinomiais podem ter. Existem diversos artigos que investigam as posições relativas de ciclos limites para o sistema (1.1.1). Um resultado geral foi obtido por Llibre e Rodrígues [37] em 2004.

Definição 1.2.2. Uma configuração de ciclos limites é um conjunto finito $C=\left\{\gamma_{1}, \cdots, \gamma_{m}\right\}$ de curvas fechadas simples tais que $\gamma_{i} \cap \gamma_{j}=\varnothing$, para todo $i \neq j$.

$O$ conjunto $C$ será chamado uma configuração de ciclos limites algébricos se as curvas fechadas $\gamma_{i}$ forem ciclos limites algébricos. Uma curva fechada será chamada algébrica se for uma componente conexa do conjunto de zeros de alguma função polinomial.

Dada uma configuração de ciclos limites $C=\left\{\gamma_{1}, \cdots, \gamma_{m}\right\}$, a curva $\gamma_{i}$ será primária se não existir nenhuma curva $\gamma_{j}$ de $C$ contida na região limitada determinada por $\gamma_{i}$.

Duas configurações de ciclos limites $C=\left\{\gamma_{1}, \cdots, \gamma_{n}\right\}$ e $C^{\prime}=\left\{\gamma_{1}^{\prime}, \cdots, \gamma_{m}^{\prime}\right\}$ são (topologicamente) equivalentes se existir um homeomorfismo $h$ aplicando $C$ em $C^{\prime}$ tal que $h\left(\cup_{i=1}^{n} \gamma_{i}\right)=\cup_{j=1}^{m} \gamma_{j}^{\prime}$. É claro que para configurações equivalentes de ciclos limites $C$ e $C^{\prime}$ temos $n=m$.

Um sistema (1.1.1) realiza a configuração de ciclos limites $C$, se o conjunto de todos os seus ciclos limites for equivalente a $C$.

Teorema 1.2.3. [15] Sejam C uma configuração de ciclos limites er o número de ciclos limites primários de $C$. Então, $C=\left\{\gamma_{1}, \cdots, \gamma_{m}\right\}$ é realizável como ciclos limites algébricos por um sistema polinomial (1.1.1) de grau menor ou igual a $2(m+r)-1$.

\subsection{A versão fraca do XVI problema de Hilbert}

Devida à dificuldade do problema original, Arnol'd [2, 4] propôs o que hoje é conhecida como a versão fraca do XVI problema de Hilbert, que de modo sucinto propõe a 
investigação do número máximo de ciclos limites que bifurcam de uma singularidade do tipo centro. Esse estudo tem possibilitado a obtenção de resultados interessantes para casos particulares do problema original.

No entanto, antes de explorarmos esta versão do problema, consideremos algumas definições e observações que nos ajudarão na sua compreensão.

Definição 1.3.1. [3] Uma 1-forma em $\mathbb{R}^{n}$ é uma função linear $\omega: \mathbb{R}^{n} \rightarrow \mathbb{R}$ tal que

$$
\omega\left(\lambda_{1} \xi_{1}+\lambda_{2} \xi_{2}\right)=\lambda_{1} \omega\left(\xi_{1}\right)+\lambda_{2} \omega\left(\xi_{2}\right)
$$

$\operatorname{com} \lambda_{1}, \lambda_{2} \in \mathbb{R}$ e $\xi_{1}, \xi_{2} \in \mathbb{R}^{n}$

Observação 1.3.2. O espaço de todas as 1-formas em $\mathbb{R}^{n}$ é um espaço vetorial de dimensão $n$ chamado de espaço dual de $\mathbb{R}^{n}$ e denotado por $\left(\mathbb{R}^{n}\right)^{*}$ cuja operação de adição é dada por

$$
\left(\omega_{1}+\omega_{2}\right)(\xi)=\omega_{1}(\xi)+\omega_{2}(\xi)
$$

e de multiplicação por escalar, por

$$
(\lambda \omega)(\xi)=\lambda \omega(\xi)
$$

Escolhamos coordenadas $x_{1}, \ldots, x_{n} \in \mathbb{R}^{n}$ e definimos as projeções (logo, transformações lineares)

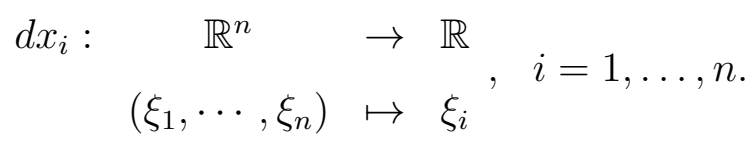

Como as $n$ projeções $d x_{i}, i=1, \ldots, n$, são linearmente independentes, formam uma base de $\left(\mathbb{R}^{n}\right)^{*}$ e, portanto, qualquer 1 -forma em $\mathbb{R}^{n}$ pode ser escrita como

$$
\omega(\xi)=\lambda_{1}(\xi) d x_{1}+\cdots+\lambda_{n}(\xi) d x_{n}, \lambda_{i}: \mathbb{R}^{n} \rightarrow \mathbb{R}
$$

Definição 1.3.3. Uma integral abeliana é a integral de linha de uma 1-forma racional em $\mathbb{R}^{2}$ ao longo de uma órbita periódica algébrica.

Definição 1.3.4. Uma curva $\gamma$ será chamada de oval se for uma curva fechada. 
Sejam $H=H(x, y)$ um polinômio em $x$ e $y$ de grau $m \geq 2$, e as curvas de nível

$$
\gamma_{h}=\left\{(x, y) \in \mathbb{R}^{2} ; H(x, y)=h\right\}
$$

formando uma família contínua de ovais $\left\{\gamma_{h}\right\}$ para $h \in(a, b)$. Consideremos a 1-forma polinomial

$$
\omega=f(x, y) d y-g(x, y) d x
$$

onde $\max (g r(f), g r(g))=n \geq 2$.

Arnol'd propôs o seguinte problema: para $m$ e $n$ inteiros fixados, encontrar o número máximo $Z(m, n)$ de zeros (isolados) das integrais abelianas $I(h)=\oint_{\gamma_{h}} \omega$.

Observação 1.3.5. A função $I(h)$ pode ser multivalente, isto é, é possível existir duas ou mais ovais sobre uma mesma curva de nível $\left\{H^{-1}(h)\right\}$.

Notemos que no problema acima devemos considerar todos os possíveis polinômios $H$ com todas as possíveis famílias de ovais $\left\{\gamma_{h}\right\}$, e arbitrárias $f$ e $g$.

A princípio esse problema parece não ter relação com o XVI problema de Hilbert. No entanto, veremos na próxima seção que a função $I(h)$, a integral abeliana, é a primeira aproximação, com respeito a $\varepsilon$ (suficientemente pequeno), para o número de ciclos limites (pelo menos localmente) para o sistema:

$$
\left\{\begin{aligned}
\dot{x} & =-\frac{\partial H}{\partial y}(x, y)+\varepsilon f(x, y), \\
\dot{y} & =\frac{\partial H}{\partial x}(x, y)+\varepsilon g(x, y),
\end{aligned}\right.
$$

onde $H, f$ e $g$ são exatamente as mesmas utilizadas para definir a integral abeliana $I(h)$.

Observemos que, se $m=n+1$, então o sistema (1.3.1) é uma forma especial do sistema (1.1.1), próximo ao sistema hamiltoniano para $\varepsilon$ suficientemente pequeno. Nesse sentido, a segunda parte do XVI problema de Hilbert é usualmente chamada de o XVI problema de Hilbert fraco (ou tangencial, ou infinitesimal), e o número $\tilde{Z}(n)=Z(n+1, n$ ) (que é igual ao número de zeros da integral abeliana) pode ser escolhido como um limitante inferior do número de Hilbert $H(n)$.

Varchenko [44] e Khovanskii [28] provaram que para $m$ e $n$ dados, o número $Z(m, n)$ 
é uniformemente limitado com respeito à escolha do polinômio $H$, da família $\left\{\gamma_{h}\right\}$ e da 1-forma $\omega$. Esse resultado é certamente importante. Porém, não nos dá informações concretas sobre o número $Z(m, n)$.

\subsection{Integrais abelianas e ciclos limites}

Nesta seção discutimos a relação entre o número de zeros das integrais abelianas e o número de ciclos limites do sistema diferencial polinomial planar correspondente.

\subsubsection{O teorema de Poincaré-Pontryagin}

Consideremos um polinômio $H(x, y)$ de grau $m$ como na seção anterior e o campo vetorial $X_{H}$ correspondente dado por

$$
\left\{\begin{aligned}
\dot{x} & =-\frac{\partial H}{\partial y}(x, y) \\
\dot{y} & =\frac{\partial H}{\partial x}(x, y)
\end{aligned}\right.
$$

Sejam $X_{H, \varepsilon}$ o sistema perturbado relacionado a $X_{H}$

$$
\left\{\begin{aligned}
\dot{x} & =-\frac{\partial H}{\partial y}(x, y)+\varepsilon f(x, y) \\
\dot{y} & =\frac{\partial H}{\partial x}(x, y)+\varepsilon g(x, y)
\end{aligned}\right.
$$

onde $f$ e $g$ são polinômios em $x$ e $y$ de graus no máximo $n$ e $\varepsilon$ um parâmetro suficientemente pequeno. Notemos que, se $\varepsilon=0$, então (1.4.2) será o sistema hamiltoniano (1.4.1) (não perturbado) associado ao polinômio $H$.

Suponhamos que exista uma família contínua de ovais $\left\{\gamma_{h}\right\} \subset H^{-1}(h)$ dependendo continuamente de um parâmetro $h \in(a, b)$. Então, definimos a integral abeliana como antes:

$$
I(h)=\oint_{\gamma_{h}} f(x, y) d y-g(x, y) d x
$$

Observação 1.4.1. Notemos que, para $h \in(a, b)$, as $\gamma_{h}$ formam um anel de ovais, e cada uma delas é órbita periódica do sistema Hamiltoniano (1.4.1). 
A questão é: quantas ovais mantêm-se e tornam-se as órbitas periódicas do sistema perturbado (1.4.2) para $\varepsilon$ suficientemente pequeno?

Se o número de tais órbitas for finito, então elas serão ciclos limites de (1.4.2).

Em outras palavras, questiona-se: é possivel encontrar um valor $h \in(a, b)$ e algumas órbitas periódicas $\Gamma_{\varepsilon}$ do sistema perturbado (1.4.2) tais que $\Gamma_{\varepsilon}$ tenda a $\gamma_{h}$ (no sentido da distância de Hausdorff) quando $\varepsilon \rightarrow 0$ ? E quantas $\Gamma_{\varepsilon}$ existem para o mesmo $h$ ?

Para responder a essa pergunta, tomemos um segmento $\sigma$, transversal a cada $\gamma_{h}$. Parametrizemos $\sigma$ pela função $H$ e denotemos por $\gamma(h, \varepsilon)$ o pedaço da órbita do sistema perturbado (1.4.2) entre o ponto inicial $h$ em $\sigma$ e o próximo ponto de interseção $P(h, \varepsilon)$ com $\sigma$, como mostra a Figura 1.2.

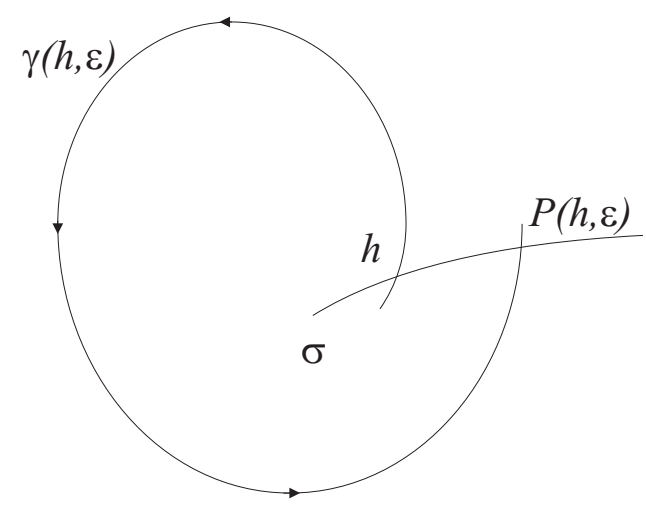

Figura 1.2: Construção da função sucessão.

A "próxima interseção" é possível para $\varepsilon$ suficientemente pequeno uma vez que $\gamma(h, \varepsilon)$ está próximo a $\gamma_{h}$.

Definição 1.4.2. A diferença $d(h, \varepsilon)=P(h, \varepsilon)-h$ é chamada de função sucessão.

Teorema 1.4.3 (Poincaré-Pontryagin). [13] Temos

$$
d(h, \varepsilon)=\varepsilon(I(h)+\varepsilon \phi(h, \varepsilon)),
$$

quando $\varepsilon \rightarrow 0$, onde $\phi(h, \varepsilon)$ é analítica e uniformemente limitada para $(h, \varepsilon)$ numa região compacta próxima a $(h, 0), h \in(a, b)$.

Demonstração: Por construção, a função sucessão é dada pela diferença da função $H$ 
entre os pontos inicial e final de $\gamma(h, \varepsilon)$, isto é,

$$
d(h, \varepsilon)=\int_{\gamma(h, \varepsilon)} d H=\left.\int_{\gamma(h, \varepsilon)}\left(\frac{\partial H}{\partial x} \frac{d x}{d t}+\frac{\partial H}{\partial y} \frac{d y}{d t}\right)\right|_{(1.4 .2)} d t
$$

(Omitimos o ponto $(x, y)$, onde as derivadas parciais são calculadas, para não carregar a notação.)

Substituindo (1.4.2) no lado direito da igualdade, obtemos:

$$
\begin{aligned}
d(h, \varepsilon) & =\left.\int_{\gamma(h, \varepsilon)}\left[\frac{\partial H}{\partial x}\left(-\frac{\partial H}{\partial y}+\varepsilon f\right)+\frac{\partial H}{\partial y}\left(\frac{\partial H}{\partial x}+\varepsilon g\right)\right]\right|_{(1.4 .2)} d t= \\
& =\left.\int_{\gamma(h, \varepsilon)}\left[-\frac{\partial H}{\partial x} \frac{\partial H}{\partial y}+\varepsilon \frac{\partial H}{\partial x} f+\frac{\partial H}{\partial y} \frac{\partial H}{\partial x}+\varepsilon \frac{\partial H}{\partial y} g\right]\right|_{(1.4 .2)} d t= \\
& =\left.\varepsilon \int_{\gamma(h, \varepsilon)}\left(\frac{\partial H}{\partial x} f+\frac{\partial H}{\partial y} g\right)\right|_{(1.4 .2)} d t .
\end{aligned}
$$

Notemos que $\gamma(h, \varepsilon)$ converge uniformemente a $\gamma_{h}$ quando $\varepsilon \rightarrow 0$, já que $\gamma_{h}$ é compacto, e que $\frac{\partial H}{\partial x} d t=d y$ e $\frac{\partial H}{\partial y} d t=-d x$ por (1.4.1). Então,

$$
d(h, \varepsilon)=\varepsilon \int_{\gamma(h, \varepsilon)}(f d y-g d x)=\varepsilon I(h)+o\left(\varepsilon^{2}\right)
$$

onde $o\left(\varepsilon^{2}\right)$ representa termos de ordem maior ou igual a 2 em $\varepsilon$.

Observação 1.4.4. O número de zeros da função sucessão é independente da escolha do segmento transversal $\sigma$, já que o consideramos parametrizado pela função $H$.

Definição 1.4.5. [43] Um ciclo limite $\Gamma$ diz-se um atrator periódico (respectivamente, repulsor periódico) (ou orbitalmente estável (respectivamente, orbitalmente instável)) quando $\Gamma$ for o $\omega$-limite (respectivamente, $\alpha$-limite) de todos os pontos numa vizinhança $V_{\Gamma}$ de $\Gamma$.

O próximo resultado caracteriza a estabilidade de um ciclo limite.

Teorema 1.4.6. [43] Sejam $D \subset \mathbb{R}^{2}$ um aberto e $\chi=(P, Q): D \rightarrow \mathbb{R}^{2}$ o campo vetorial relacionado à equação (1.1.1). Sejam $\Gamma$ uma órbita periódica de $\chi$ de período $T, p \in \Gamma$ e $\sigma$ 
uma seção transversal de $\Gamma$ em $p$. Definamos a função sucessão d de $\Gamma$ numa vizinhança $\sigma_{0}$ de p. Então,

$$
d^{\prime}(p)=\exp \left[\int_{0}^{T} \operatorname{div} \chi(\gamma(t)) d t\right]
$$

onde $\gamma(t)$ é a parametrização de $\Gamma$ e $\operatorname{div} \chi(x, y)=\frac{\partial P}{\partial x}+\frac{\partial Q}{\partial y}$.

Em particular, se $\int_{0}^{T} \operatorname{div} \chi(\Gamma(t)) d t<0$, então $\Gamma$ é estável, e se $\int_{0}^{T} \operatorname{div} \chi(\Gamma(t)) d t>0$, $\Gamma$ é instável.

Com a notação do Teorema 1.4.6, definimos a hiperbolicidade de um ciclo limite.

Definição 1.4.7. [15] Um ciclo limite $\Gamma$ de (1.1.1) diz-se hiperbólico se

$$
\int_{0}^{T}\left(\frac{\partial P}{\partial x}+\frac{\partial Q}{\partial y}\right) d t \neq 0
$$

onde $T$ é o período de $\Gamma$.

Definição 1.4.8. Se existirem $h^{*} \in(a, b) e \varepsilon^{*}>0$ tais que $X_{H, \varepsilon}$ tenha um ciclo limite $\Gamma_{\varepsilon}$ para $0<|\varepsilon|<\varepsilon^{*}$ e $\Gamma_{\varepsilon}$ tenda a $\gamma_{h^{*}}$ quando $\varepsilon \rightarrow 0$, então diremos que $\Gamma_{\varepsilon}$ bifurca-se de $\gamma_{h^{*}}$. Diremos que um ciclo limite $\Gamma$ de $X_{H, \varepsilon}$ bifurca-se do anel $\bigcup_{h \in(a, b)} \gamma_{h}$ de $X_{H}$, se existir $h \in(a, b)$ tal que $\Gamma$ bifurca-se de $\gamma_{h}$.

O próximo resultado fornece uma caracterização do número máximo de ciclos limites bifurcados de um centro com relação ao número máximo de zeros da integral abeliana correspondente.

Teorema 1.4.9. [13] Suponhamos que $I(h)$ não seja identicamente nula para $h \in(a, b)$. Então, valem as seguintes afirmações.

1. Se $X_{H, \varepsilon}$ tiver um ciclo limite bifurcando-se de $\gamma_{h^{*}}$, então $I\left(h^{*}\right)=0$;

2. Se existir $h^{*} \in(a, b)$ tal que $I\left(h^{*}\right)=0$ e $I^{\prime}\left(h^{*}\right) \neq 0$, então $X_{H, \varepsilon}$ terá um único ciclo limite bifurcando-se de $\gamma_{h^{*}}$. Além disso, esse ciclo limite é hiperbólico;

3. Se existir $h^{*} \in(a, b)$ tal que $I\left(h^{*}\right)=I^{\prime}\left(h^{*}\right)=\cdots=I^{(k-1)}\left(h^{*}\right)=0$ e $I^{(k)}\left(h^{*}\right) \neq 0$, então $X_{H, \varepsilon}$ terá no máximo $k$ ciclos limites bifurcando-se da mesma $\gamma_{h^{*}}$, levando em consideração a multiplicidade dos ciclos limites; 
4. O número total de ciclos limites de $X_{H, \varepsilon}$ (contando as multiplicidades), bifurcandose do anel $\bigcup_{h \in(a, b)} \gamma_{h}$ de $X_{H}$, é limitado pelo número máximo de zeros isolados da integral abeliana $I(h)$, para $h \in(a, b)$ (levando em consideração as multiplicidades).

\section{Demonstração:}

1. Suponhamos que um ciclo limite $\Gamma_{\varepsilon}$ de $X_{H, \varepsilon}$ bifurca-se de $\gamma_{h^{*}}$. Pela Definição 1.4.8, segue que existe um $\varepsilon^{*}>0$ e uma sequência $\left\{h_{\varepsilon}\right\}_{\varepsilon}$ tais que $h_{\varepsilon} \rightarrow h^{*}$, quando $\varepsilon \rightarrow 0$, e $d\left(h_{\varepsilon}, \varepsilon\right)=0$, com $0<|\varepsilon|<\varepsilon^{*}$.

Assim, pelo Teorema 1.4.3, temos

$$
d\left(h_{\varepsilon}, \varepsilon\right)=\varepsilon\left(I\left(h_{\varepsilon}\right)+\varepsilon \phi\left(h_{\varepsilon}, \varepsilon\right)\right)=0, \quad 0<|\varepsilon|<\varepsilon^{*} .
$$

Logo, fazendo $\varepsilon \rightarrow 0$, teremos $h_{\varepsilon} \rightarrow h^{*}$ e, então, $I\left(h^{*}\right)=0$.

2. Suponhamos que exista $h^{*} \in(a, b)$ tal que $I\left(h^{*}\right)=0$ e $I^{\prime}\left(h^{*}\right) \neq 0$. Já que os ciclos limites são considerados para $\varepsilon \neq 0$ suficientemente pequeno, ao invés da função sucessão, estudemos os zeros da função $\tilde{d}(h, \varepsilon)=\frac{d(h, \varepsilon)}{\varepsilon}$.

Pelo Teorema 1.4.3, temos

$$
\tilde{d}(h, \varepsilon)=I(h)+\varepsilon \phi(h, \varepsilon),
$$

onde $\phi$ é analítica e uniformemente limitada em uma região compacta próxima a $\left(h^{*}, 0\right)$.

Notemos que

$$
\tilde{d}\left(h^{*}, 0\right)=I\left(h^{*}\right) \stackrel{h i p}{=} 0
$$

e que

$$
\frac{\partial}{\partial h} \tilde{d}(h, \varepsilon)=I^{\prime}(h)+\varepsilon \frac{\partial}{\partial h} \phi(h, \varepsilon) .
$$

Isto implica

$$
\frac{\partial}{\partial h} \tilde{d}\left(h^{*}, 0\right)=I^{\prime}\left(h^{*}\right) \neq 0 .
$$


Pelo Teorema da Função Implícita, existem $\varepsilon^{*}>0, \eta^{*}>0$ e uma única função $h=h(\varepsilon)$ definida em

$$
\mathcal{U}^{*}=\left\{(h, \varepsilon) ;\left|h-h^{*}\right| \leq \eta^{*},|\varepsilon| \leq \varepsilon^{*}\right\}
$$

tais que $h(0)=h^{*}$ e $\tilde{d}(h(\varepsilon), \varepsilon) \equiv 0$ para $(h, \varepsilon) \in \mathcal{U}^{*}$. Consequentemente, a única $h(\varepsilon)$ fornece um único ciclo limite $\Gamma_{\varepsilon}$ do sistema (1.4.2) para cada $\varepsilon$ suficientemente pequeno.

Agora precisamos mostrar que esse ciclo limite é hiperbólico (para $\varepsilon$ suficientemente pequeno).

Escrevemos $I(h)$ em (1.4.3) como $I_{1}(h)+I_{2}(h)$, onde

$$
I_{1}(h)=\oint_{\gamma_{h}} f(x, y) d y \quad \text { e } \quad I_{2}(h)=-\oint_{\gamma_{h}} g(x, y) d x
$$

Na primeira integral consideremos $x=x(y, h)$ como função de $y$ e $h$ e, ao longo de $\gamma_{h}$, temos

$$
H(x, y)=h \Rightarrow \frac{\partial H}{\partial x} \frac{\partial x}{\partial h}=1 \quad \text { e } \quad d y=\frac{\partial H}{\partial x} d t
$$

Isso nos dá

$$
I_{1}^{\prime}(h)=\oint_{\gamma_{h}} \frac{\partial f}{\partial x} \frac{\partial x}{\partial h} d y=\oint_{\gamma_{h}} \frac{\partial f}{\partial x} \frac{\partial x}{\partial h} \frac{\partial H}{\partial x} d t=\oint_{\gamma_{h}} \frac{\partial f}{\partial x} d t .
$$

Analogamente, consideremos $y=y(x, h)$ uma função de $x$ e $h$ na segunda integral e, ao longo de $\gamma_{h}$, temos

$$
H(x, y)=h \Rightarrow \frac{\partial H}{\partial y} \frac{\partial y}{\partial h}=1 \quad \text { e } \quad d x=-\frac{\partial H}{\partial y} d t
$$

Daí,

$$
I_{2}^{\prime}(h)=-\oint_{\gamma_{h}} \frac{\partial g}{\partial y} \frac{\partial y}{\partial h} d x=\oint_{\gamma_{h}} \frac{\partial g}{\partial y} \frac{\partial y}{\partial h} \frac{\partial H}{\partial y} d t=\oint_{\gamma_{h}} \frac{\partial g}{\partial y} d t
$$


Consequentemente,

$$
\oint_{\gamma_{h^{*}}}\left(\frac{\partial f}{\partial x}+\frac{\partial g}{\partial y}\right) d t=I^{\prime}\left(h^{*}\right) \stackrel{\text { hip }}{\neq} 0
$$

que implica

$$
\begin{aligned}
\oint_{\Gamma \varepsilon} \operatorname{tr}(1.4 .2) d t & =\oint_{\Gamma \varepsilon}\left(\frac{\partial^{2} H}{\partial y \partial x}+\varepsilon \frac{\partial f}{\partial x}-\frac{\partial^{2} H}{\partial x \partial y}+\varepsilon \frac{\partial g}{\partial y}\right) d t \stackrel{\text { T. Schawrz }}{=} \\
& =\varepsilon \oint_{\Gamma_{\varepsilon}}\left(\frac{\partial f}{\partial x}+\frac{\partial g}{\partial y}\right) d t \neq 0, \quad 0<|\varepsilon| \ll 1
\end{aligned}
$$

já que $\Gamma_{\varepsilon} \rightarrow \gamma_{h^{*}}$ quando $\varepsilon \rightarrow 0$, e a hiperbolicidade de $\Gamma_{\varepsilon}$ segue.

3. Assumamos que exista $h^{*} \in(a, b)$ tal que $I\left(h^{*}\right)=I^{\prime}\left(h^{*}\right)=\cdots=I^{(k-1)}\left(h^{*}\right)=0$ e $I^{(k)}\left(h^{*}\right) \neq 0$. Precisamos provar que existem $\delta>0$ e $\eta>0$ tais que, para qualquer $(h, \varepsilon) \in \mathcal{U}=\left\{(h, \varepsilon) ; \quad\left|h-h^{*}\right|<\eta, \quad|\varepsilon|<\delta\right\}$, a função sucessão $d(h, \varepsilon)$ tenha no máximo $k$ zeros em $h$, levando em consideração suas multiplicidades.

Suponhamos que isso não aconteça. Então, para qualquer inteiro $j$, existem $\varepsilon_{j}>0$ e $\eta_{j}>0$, com $\varepsilon_{j} \rightarrow 0$ e $\eta_{j} \rightarrow 0$ quando $j \rightarrow \infty$, tais que para quaisquer $\varepsilon_{j}$ a função $\frac{d\left(h, \varepsilon_{j}\right)}{\varepsilon_{j}}$ tenha no mínimo $k+1$ zeros para $\left|h-h^{*}\right|<\eta_{j}$.

Usando o Teorema de Rolle (sucessivas vezes), encontramos $h_{j}$ tal que $\left|h_{j}-h^{*}\right|<\eta_{j}$ e

$$
\left(\frac{d\left(h_{j}, \varepsilon_{j}\right)}{\varepsilon_{j}}\right)^{(k)}=I^{(k)}\left(h_{j}\right)+\varepsilon_{j} \frac{\partial^{k}}{\partial h^{k}} \phi\left(h_{j}, \varepsilon_{j}\right)=0 .
$$

Fazendo $j \rightarrow \infty$, teremos $\varepsilon_{j} \rightarrow 0$ e, assim, $I^{(k)}\left(h^{*}\right)=0$, o que é uma contradição.

Portanto, existem no máximo $k$ ciclos limites bifurcando-se de $\gamma_{h^{*}}$.

4. Esta afirmação é uma consequência das três anteriores. De fato, para $\delta>0$ suficientemente pequeno, podemos considerar o número de ciclos limites bifurcandose de $\gamma_{h}$, para $h \in[a+\delta, b-\delta]$ e $\varepsilon$ suficientemente pequeno. Vimos que esse número é uniformemente limitado. Tomando o máximo desse número quando $\delta \rightarrow 0$, conseguimos a ciclicidade do anel periódico.

Exemplo 1.4.10. Consideremos a equação de Van der Pol

$$
\ddot{u}+\varepsilon\left(u^{2}-1\right) \dot{u}+u=0,
$$


que é equivalente ao sistema

$$
\left\{\begin{array}{l}
\dot{x}=y \\
\dot{y}=-x+\varepsilon\left(1-x^{2}\right) y .
\end{array}\right.
$$

Notemos que para $\varepsilon=0$, o sistema (1.4.4) é hamiltoniano cuja família contínua de ovais é

$$
\gamma_{h}=\left\{(x, y) \in \mathbb{R}^{2} ; H(x, y)=x^{2}+y^{2}=h, h>0\right\}
$$

Usando coordenadas polares $x=h \cos \theta$ e $y=h \operatorname{sen} \theta$, e observando que a orientação de $\gamma_{h}$ está no sentido horário, temos:

$$
\begin{aligned}
I(h)=\oint_{\gamma_{h}} & \left(1-x^{2}\right) y d x=\int_{0}^{2 \pi}\left(1-h^{2} \cos ^{2} \theta\right)(h \operatorname{sen} \theta)(-h \operatorname{sen} \theta d \theta)= \\
& =-\int_{0}^{2 \pi}\left(1-h^{2} \cos ^{2} \theta\right)\left(h^{2} \operatorname{sen}^{2} \theta\right) d \theta= \\
& =-\int_{0}^{2 \pi}\left(h^{2} \operatorname{sen}^{2} \theta-h^{4} \operatorname{sen}^{2} \theta \cos ^{2} \theta\right) d \theta= \\
& =-\int_{0}^{2 \pi} h^{2} \operatorname{sen}^{2} \theta d \theta+\int_{0}^{2 \pi} h^{4}(\operatorname{sen} \theta \cos \theta)^{2} d \theta .
\end{aligned}
$$

Fazendo a substituição $1-\cos 2 \theta=2 \operatorname{sen}^{2} \theta$, obtemos:

$$
I(h)=-h^{2} \int_{0}^{2 \pi} \frac{1-\cos 2 \theta}{2} d \theta+\frac{h^{4}}{2} \int_{0}^{2 \pi} \frac{1-\cos 4 \theta}{2} d \theta=\pi h^{2}\left(\frac{h^{2}}{4}-1\right) .
$$

Notemos que $I(h)=0$ se, e somente se,

$$
h=0 \quad \text { ou } \quad \frac{h^{2}}{4}-1=0 \Leftrightarrow h= \pm 2 .
$$

O valor $h^{*}=0$ corresponde à singularidade do sistema e $h^{*}=2$ é o único zero positivo de $I(h)$.

Por outro lado, vemos que

$$
I^{\prime}(h)=2 \pi h\left(\frac{h^{2}}{2}-1\right)
$$


implica

$$
I^{\prime}(2)=4 \pi\left(\frac{2^{2}}{2}-1\right)=4 \pi \neq 0 .
$$

Usando o Teorema 1.4 .9 concluímos que, para $\varepsilon$ suficientemente pequeno, o sistema (1.4.4) possui um único ciclo limite hiperbólico que tende ao círculo de raio $\sqrt{2}$ quando $\varepsilon \rightarrow 0$.

Observação 1.4.11. [13] Se ambos os sistemas, hamiltoniano e perturbado, independerem de parâmetros, como no Exemplo 1.4.10, então o Teorema 1.4 .9 assegurará resultados mais definidos. No entanto, é comum encontrarmos sistemas nos quais funções com parâmetros, e/ou o sistema hamiltoniano, dependam de outros parâmetros. Esses parâmetros podem impossibilitar a utilização do Teorema 1.4.9.

Se as perturbações dependerem de parâmetros, os zeros de $I(h)$ também dependerão dos mesmos parâmetros. Esses podem tender aos extremos do intervalo $(a, b)$, correspondendo a valores críticos de $H$. Em tais valores o Teorema da Função Implícita não pode, em geral, ser aplicado. Se um dos extremos, digamos $a$, corresponder ao centro de $X_{H}$, então $I(h)$ poderá ser estendida analiticamente ao valor $a$, e $I(h)$ terá no máximo um número finito de zeros próximos de $a$; consequentemente, o item $(c)$ do Teorema 1.4.9 pode ser estendido a $[a, b)$. Por outro lado, se um extremo, digamos $b$, corresponder a um policiclo (órbita homoclínica ou heteroclínica) de $X_{H}$, as melhores conclusões serão as seguintes. O item $(c)$ poderá ser estendido a $[a, b]$ se $b$ corresponder ao laço homoclínico e, em geral, certamente não se poderá estender a $[a, b]$ se $b$ corresponder à conexão heteroclínica. Enfim, se $b=\infty$ (o anel tende ao infinito), poderíamos fazer conclusões sobre o número de ciclos limites somente em regiões compactas do anel.

Se $H=H_{\nu}$ depender de algum parâmetro $\nu$, então para alguns valores especiais, digamos $\nu^{*}, H_{\nu^{*}}$ poderá ser degenerado, por exemplo ter algumas simetrias. Como Iliev [26] explicou, a degenerescência leva a um limitante menor do que esperado para o número de zeros de $I(h)$. Além disso, a função $I(h)$ (mesmo no caso em que $I(h)$ não for identicamente nula) nunca pode gerar o número máximo de zeros da função sucessão $d(h, \varepsilon)$ para todas as classes de perturbações. Nesse caso, ordens mais elevadas (em $\varepsilon$ ) de aproximações para $d(h, \varepsilon)$ são necessárias, como no estudo da ciclicidade do anel periódico para 
$X_{H} \in Q_{3}^{H} \cap\left\{Q_{3}^{R} \cup Q_{3}^{L V}\right\}$ (isto é, $X_{H}$ um sistema hamiltoniano do tipo reversível ou LotkaVolterra); ou os parâmetros que quebram a simetria de $H$ e os parâmetros de perturbação deveriam ser considerados juntos em espaços de dimensões maiores para dar uma "parte principal" da função sucessão.

Observação 1.4.12. Os Teoremas 1.4.3 e 1.4.9 foram provados para sistemas polinomiais hamiltonianos (1.4.1) e sua perturbação polinomial (1.4.2), mas essencialmente podem ser provados para campos vetoriais analíticos.

\subsection{Estimativa do número de zeros das integrais abelianas}

Vimos que para estudar a versão fraca do XVI problema de Hilbert usando integrais abelianas é fundamental estimar seu número de zeros. Li e Zhang [32] observaram que a monotonicidade da razão de duas integrais abelianas é um dado importante para determinar o número de zeros de $I(h)$. Existem muitos métodos para estudar essa monotonicidade. Três deles são:

1. a utilização de equações de Picard-Fuchs;

2. a utilização da fórmula de Green;

3. estimativas diretas da integral $I_{2}^{\prime}(h) I_{1}(h)-I_{1}^{\prime}(h) I_{2}(h)$.

Cada um desses métodos é aplicável a alguns casos especiais. No entanto, é necessário repetir todo o processo de cálculos para cada problema individual. Em [32], o objetivo dos autores foi dar um novo método que seja mais direto, eficiente para problemas mais amplos e conveniente para ser usado. Em suma, esse método constrói funções auxiliares, definidas a partir da função hamiltoniana e das integrais abelianas, cujas propriedades resultarão na monotonicidade da razão de duas integrais abelianas.

Nesta seção introduziremos o método baseado na equação de Picard-Fuchs para estimar o número de zeros da integral abeliana $I(h)$.

Após esse estudo, faremos a abordagem para um caso particular da função hamiltoniana proposta em [13]. Ressaltamos que o sistema a ser estudado é conhecido como sistema de Bogdanov-Takens. 


\subsubsection{O método baseado na equação de Picard-Fuchs}

Consideremos a função hamiltoniana elíptica de grau 3 na forma

$$
H(x, y)=\frac{y^{2}}{2}-\frac{x^{3}}{3}+x
$$

com a família contínua de ovais

$$
\gamma_{h}=\left\{(x, y) ; \quad H(x, y)=h, \quad-\frac{2}{3} \leq h \leq \frac{2}{3}\right\}
$$

como mostra a Figura 1.3.

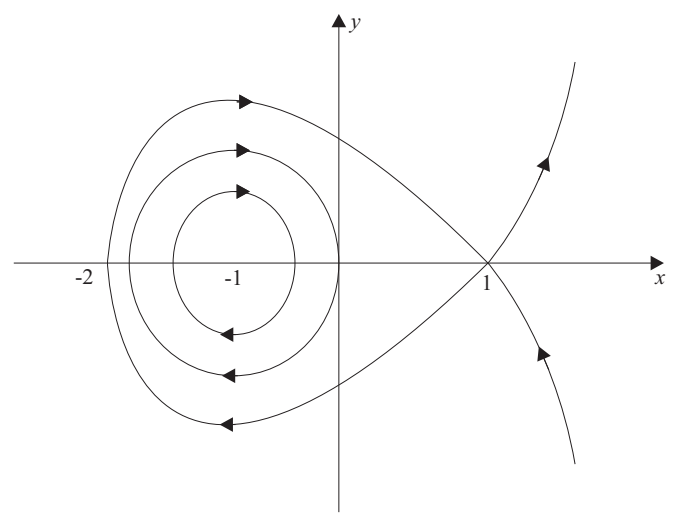

Figura 1.3: Curvas de nível de (1.5.1).

Denotemos o sistema hamiltoniano correspondente por $X_{H}$, a saber

$$
\left\{\begin{array}{l}
\dot{x}=y \\
\dot{y}=x^{2}-1
\end{array}\right.
$$

Quando $h \rightarrow-\frac{2}{3}$, a oval $\gamma_{h}$ reduz-se ao centro de $X_{H}$ em $(-1,0)$, enquanto que se $h \rightarrow \frac{2}{3}$, a oval $\gamma_{h}$ resultará no laço homoclínico com sela em $(1,0)$. Consideremos o sistema perturbado $X_{H, \varepsilon}$ de $X_{H}$ da seguinte forma

$$
\left\{\begin{array}{l}
\dot{x}=y \\
\dot{y}=x^{2}-1+\varepsilon(\alpha+x) y
\end{array}\right.
$$

onde $\alpha$ é uma constante e $\varepsilon$ é um parâmetro suficientemente pequeno. A integral abeliana 
correspondente é

$$
I(h)=\alpha I_{0}(h)+I_{1}(h), \text { onde } I_{j}(h)=\oint_{\gamma_{h}} x^{j} y d x, \quad j=0,1,2, \ldots
$$

Desde que a orientação de $\gamma_{h}$ está no sentido horário, usando a fórmula de Green vemos que $I_{0}(h)$ é a área da região delimitada por $\gamma_{h}$ e, consequentemente, $I_{0}(h)>0$ para $h>-\frac{2}{3}$. Sejam $\left(\xi_{h}, 0\right)$ e $\left(\eta_{h}, 0\right), \operatorname{com} \xi_{h}<-1<\eta_{h}<1$, os dois pontos de interseção de $\gamma_{h}$ com o eixo- $x$. Então,

$$
I_{j}(h)=2 \int_{\xi_{h}}^{\eta_{h}} x^{j} y d x .
$$

Seja $H(x, y)=h$ com $y=y(x, h)>0$. Diferenciando $H$ em relação a $h$, obtemos

$$
\frac{\partial}{\partial y} H(x, y) \cdot \frac{\partial}{\partial h} y(x, h)=1
$$

o que implica que

$$
\frac{\partial}{\partial h} y(x, h)=\frac{1}{\frac{\partial}{\partial y} H(x, y)}=\frac{1}{y(x, h)} .
$$

Logo,

$$
I_{j}^{\prime}(h)=2 \int_{\xi_{h}}^{\eta_{h}} \frac{x^{j}}{y(x, h)} d x .
$$

Lema 1.5.1. Nas condições acima, temos

$$
\lim _{h \rightarrow-\frac{2}{3}} \frac{I_{1}(h)}{I_{0}(h)}=-1 .
$$

Demonstração: Seja $f(x)$ a função tal que $f^{\prime}(x)=x y$, isto é, $f$ é a primitiva de $\int_{\xi_{h}}^{\eta_{h}} x y d x$, e seja $g(x)$ a primitiva de $\int_{\xi_{h}}^{\eta_{h}} y d x$, para cada $h$ fixado.

Então, como $f$ e $g$ são contínuas em $\left[\xi_{h}, \eta_{h}\right]$ e deriváveis em $\left(\xi_{h}, \eta_{h}\right)$, segue do Teorema de Cauchy que existe $x^{*} \in\left(\xi_{h}, \eta_{h}\right) \subset[-2,1]$ tal que

$$
\frac{f\left(\eta_{h}\right)-f\left(\xi_{h}\right)}{g\left(\eta_{h}\right)-g\left(\xi_{h}\right)}=\frac{f^{\prime}\left(x^{*}\right)}{g^{\prime}\left(x^{*}\right)}
$$

$\operatorname{Mas} f^{\prime}\left(x^{*}\right)=x^{*} y\left(x^{*}, h\right)$ e $g^{\prime}\left(x^{*}\right)=y\left(x^{*}, h\right), \operatorname{com} h$ fixado. Assim, $\frac{f^{\prime}\left(x^{*}\right)}{g^{\prime}\left(x^{*}\right)}=x^{*}$. 
Logo,

$$
\begin{aligned}
\int_{\xi_{h}}^{\eta_{h}} x y d x & =f\left(\eta_{h}\right)-f\left(\xi_{h}\right) \stackrel{(1.5 .6)}{=} \\
& =x^{*}\left[g\left(\eta_{h}\right)-g\left(\xi_{h}\right)\right]= \\
& =x^{*} \int_{\xi_{h}}^{\eta_{h}} y d x .
\end{aligned}
$$

Portanto,

$$
\lim _{h \rightarrow-\frac{2}{3}} \frac{I_{1}(h)}{I_{0}(h)} \stackrel{(1.5 .7)}{=} \lim _{h \rightarrow-\frac{2}{3}} x^{*}=-1 .
$$

Assim, se definirmos a função

$$
P(h)= \begin{cases}\frac{I_{1}(h)}{I_{0}(h)}, & h \in\left(-\frac{2}{3}, \frac{2}{3}\right], \\ -1, & h=-\frac{2}{3},\end{cases}
$$

teremos de (1.5.4) que

$$
I(h)=I_{0}(h)(\alpha+P(h)) .
$$

No que se segue, provaremos que $P^{\prime}(h)>0$ para $h \in\left(-\frac{2}{3}, \frac{2}{3}\right)$, implicando que $P(h)$ seja crescente e, assim, que $I(h)$ tenha no máximo um zero.

Lema 1.5.2. As integrais abelianas $I_{0}(h)$ e $I_{1}(h)$ satisfazem a seguinte equação de PicardFuchs:

$$
\left(9 h^{2}-4\right) \frac{d}{d h}\left(\begin{array}{c}
I_{0}(h) \\
I_{1}(h)
\end{array}\right)=\left(\begin{array}{cc}
\frac{15}{2} h & 7 \\
5 & \frac{21}{2} h
\end{array}\right)\left(\begin{array}{c}
I_{0}(h) \\
I_{1}(h)
\end{array}\right)
$$

Demonstração: Usando (1.5.5) e o fato de que $y^{2}=2 h+\frac{2}{3} x^{3}-2 x$ ao longo de $\gamma_{h}$, obtemos:

$$
\begin{aligned}
I_{j}(h) & =\oint_{\gamma_{h}} x^{j} y d x=\oint_{\gamma_{h}} \frac{x^{j} y^{2}}{y} d x=\oint_{\gamma_{h}} \frac{1}{y} x^{j}\left(2 h+\frac{2}{3} x^{3}-2 x\right) d x= \\
& =2 h \oint_{\gamma_{h}} \frac{x^{j}}{y} d x+\frac{2}{3} \oint_{\gamma_{h}} \frac{x^{j+3}}{y} d x-2 \oint_{\gamma_{h}} \frac{x^{j+1}}{y} d x= \\
& =2 h I_{j}^{\prime}(h)+\frac{2}{3} I_{j+3}^{\prime}(h)-2 I_{j+1}^{\prime}(h) .
\end{aligned}
$$


Por outro lado, usando a fórmula da integração por partes e o fato que $y d y=\left(x^{2}-1\right) d x$ ao longo de $\gamma_{h}$, obtemos:

$$
\begin{aligned}
I_{j}(h) & =2 \int_{\xi_{h}}^{\eta_{h}} x^{j} y d x=2\left[\left.\frac{1}{j+1}\left(x^{j+1} y\right)\right|_{\xi_{h}} ^{\eta_{h}}-\frac{1}{j+1} \int_{\xi_{h}}^{\eta_{h}} x^{j+1} d y\right]= \\
& =2\left[-\frac{1}{j+1}\left(\int_{\xi_{h}}^{\eta_{h}}-\frac{x^{j+1}}{y} d x+\int_{\xi_{h}}^{\eta_{h}} \frac{x^{j+3}}{y} d x\right)\right]= \\
& =\frac{1}{j+1}\left[2 \int_{\xi_{h}}^{\eta_{h}} \frac{x^{j+1}}{y} d x-2 \int_{\xi_{h}}^{\eta_{h}} \frac{x^{j+3}}{y} d x\right]= \\
& =\frac{1}{j+1}\left(I_{j+1}^{\prime}(h)-I_{j+3}^{\prime}(h)\right) .
\end{aligned}
$$

Removendo $I_{j+3}^{\prime}(h)$ de (1.5.11) e (1.5.12), temos:

$$
(2 j+5) I_{j}(h)=6 h I_{j}^{\prime}(h)-4 I_{j+1}^{\prime}(h) .
$$

Tomando $j=0,1$, temos:

$$
\left\{\begin{array}{l}
5 I_{0}(h)=6 h I_{0}^{\prime}(h)-4 I_{1}^{\prime}(h), \\
7 I_{1}(h)=6 h I_{1}^{\prime}(h)-4 I_{2}^{\prime}(h) .
\end{array}\right.
$$

Notemos que, ao longo de $\gamma_{h}$, vale $y^{2} d y=\left(1-x^{2}\right) y d x$. Assim,

$$
\begin{gathered}
y^{2} d y=y d x-x^{2} y d x \Rightarrow \int_{\xi_{h}}^{\eta_{h}} y^{2} d y=\int_{\xi_{h}}^{\eta_{h}} y d x-\int_{\xi_{h}}^{\eta_{h}} x^{2} y d x \Rightarrow \\
\Rightarrow 0=2 \int_{\xi_{h}}^{\eta_{h}} y d x-2 \int_{\xi_{h}}^{\eta_{h}} x^{2} y d x= \\
=I_{0}(h)-I_{2}(h) \Rightarrow I_{0}(h)=I_{2}(h) .
\end{gathered}
$$

Usando $I_{0}^{\prime}(h)$ ao invés de $I_{2}^{\prime}(h)$ em (1.5.13), temos

$$
\left\{\begin{array}{l}
5 I_{0}(h)=6 h I_{0}^{\prime}(h)-4 I_{1}^{\prime}(h), \\
7 I_{1}(h)=6 h I_{1}^{\prime}(h)-4 I_{0}^{\prime}(h),
\end{array}\right.
$$

e resolvendo em $I_{0}^{\prime}(h)$ e $I_{1}^{\prime}(h)$ obtemos (1.5.10).

Teorema 1.5.3. A função $P(h)$ definida em (1.5.8) é estritamente crescente para 
$h \in\left(-\frac{2}{3}, \frac{2}{3}\right)$.

Demonstração: Por (1.5.8), temos

$$
P^{\prime}(h)=\frac{I_{1}^{\prime}(h) I_{0}(h)-I_{1}(h) I_{0}^{\prime}(h)}{\left(I_{0}(h)\right)^{2}}=\frac{I_{1}^{\prime}(h)}{I_{0}(h)}-\frac{I_{0}^{\prime}(h)}{I_{0}(h)} P(h) .
$$

Substituindo (1.5.10) na igualdade acima, obtemos

$$
\left(9 h^{2}-4\right) P^{\prime}(h)=-7(P(h))^{2}+3 h P(h)+5,
$$

que é uma equação de Ricatti e é equivalente ao sistema

$$
\left\{\begin{array}{l}
\dot{h}=9 h^{2}-4 \\
\dot{P}=-7 P^{2}+3 h P+5 .
\end{array}\right.
$$

Chamaremos de $\Gamma$ as curvas integrais do sistema (1.5.15), o qual tem $\left\{h= \pm \frac{2}{3}\right\}$ como conjuntos invariantes e todas as quatro singularidades estão localizadas sobre essas retas: uma sela $S_{-}=\left(-\frac{2}{3},-1\right)$ e um nó $N_{-}=\left(\frac{2}{3},-\frac{5}{7}\right)$ no semiplano inferior, e uma sela $S_{+}=\left(\frac{2}{3}, 1\right)$ e um nó $N_{+}=\left(-\frac{2}{3}, \frac{5}{7}\right)$ no semiplano superior.

A definição de $P$ em (1.5.8) mostra que o gráfico da função $P(h)$ é a variedade estável da sela $S_{-}$. De fato, seja $B=\left\{P \leq 0,-\frac{2}{3} \leq h \leq \frac{2}{3}\right\}$. Notemos a existência de uma única órbita de (1.5.15) no interior de $B$ tendo $S_{-}$como ponto $\omega$-limite. Essa órbita é a separatriz estável $\Gamma$ de $S_{-}$, que tende a $N_{-}$quanto $t \rightarrow-\infty$.

Como $P(h) \rightarrow-1$ quando $h \rightarrow-\frac{2}{3}$, segue, pela unicidade de soluções, que o gráfico de $P(h)$ é igual a $\Gamma$ (implicando que $P(h) \rightarrow-\frac{5}{7}$ quando $h \rightarrow \frac{2}{3}$ ), como mostra a Figura 1.4 .

Por outro lado, da segunda equação de (1.5.15) vemos que a isóclina horizontal é dada pela hipérbole de duas folhas $Q_{ \pm}(h)$, onde

$$
Q_{ \pm}(h)=\frac{3 h \pm \sqrt{9 h^{2}+140}}{14}
$$

As duas folhas dividem a faixa $\left\{(h, P) ;-\frac{2}{3}<h<\frac{2}{3}\right\}$ em três regiões, e o campo vetorial aponta para baixo nas regiões superior e inferior, e aponta para cima na região 


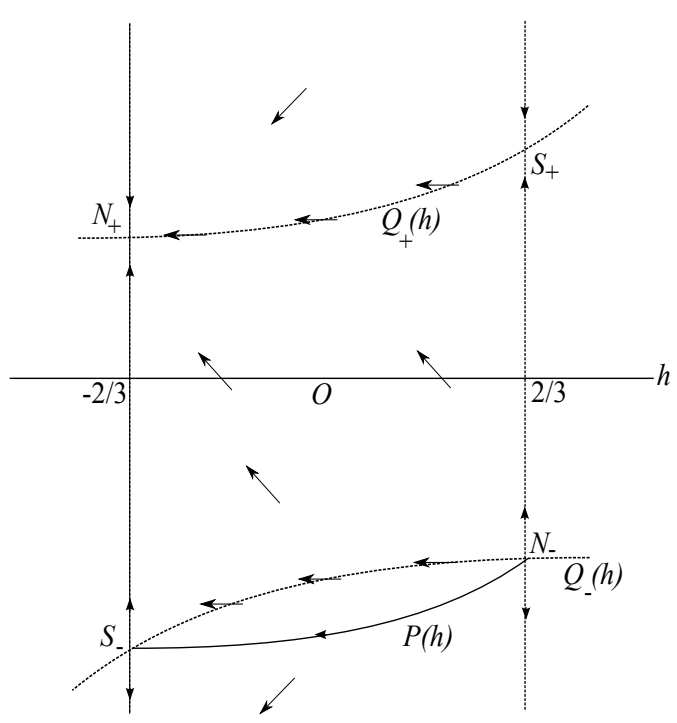

Figura 1.4: O comportamento do campo vetorial (1.5.15) e da função $P(h)$.

intermediária.

O objetivo agora é calcular a inclinação de $P(h)$ no ponto $h=-\frac{2}{3}$. Para isso, determinamos a direção característica da variedade estável da sela $S_{-}$, seguindo as ideias descritas em [48] (pp. 53-60).

Por meio de uma translação no sistema (1.5.15) de forma que a sela $S_{-}$esteja na origem, obtemos um novo sistema cuja parte linear é dada por

$$
\left\{\begin{array}{l}
\dot{h}=X(h, P)=-12 h, \\
\dot{P}=Y(h, P)=-3 h+12 P .
\end{array}\right.
$$

Considerando as coordenadas polares $h=r \cos \theta$ e $P=r \operatorname{sen} \theta$ e definindo

$$
G(\theta)=\cos \theta Y(\cos \theta, \operatorname{sen} \theta)-\operatorname{sen} \theta X(\cos \theta, \operatorname{sen} \theta)
$$

a equação $G(\theta)=0$ é chamada a equação característica para o sistema (1.5.16). Voltando às coordenadas $(h, P)$, vemos que a equação característica de (1.5.16) é dada pela reta invariante $-3 h+24 P=0$, cujo coeficiente angular é $\frac{1}{8}$.

Logo, a inclinação de $P(h)$ no ponto $S_{-}$é $P^{\prime}\left(-\frac{2}{3}\right)=\frac{1}{8}$, enquanto que a inclinação de $Q_{-}(h)$ no mesmo ponto é $\frac{1}{4}$. Consequentemente, a curva $P(h)$ está localizada abaixo de $Q_{-}$próximo a $S_{-}$. Mas, como o campo vetorial é transversal a $Q_{-}(h)$ e é direcionado à 
esquerda, a órbita $\Gamma$ não pode cruzar $Q_{-}(h)$ para $t \rightarrow-\infty$ : a órbita $\Gamma$ (gráfico de $P(h)$ ) está inteiramente situada em $B$, abaixo de $Q_{-}(h)$.

Isso implica que $P^{\prime}(h)=\frac{d P}{d t} / \frac{d h}{d t}>0$, já que $\frac{d P}{d t}<0$ abaixo de $Q_{-}(h)$ e $\frac{d h}{d t}<0$ para todo $h \in\left(-\frac{2}{3}, \frac{2}{3}\right)$.

Observação 1.5.4. Uma equação Fuchsiana é aquela que possui somente pontos singulares regulares, no sentido definido em [43], pp. 143-162. Uma vez que a equação (1.5.14) possui somente pontos singulares regulares em $h= \pm \frac{2}{3}$, ela é do tipo Fuchsiana. Uma equação Fuchsiana será dita ser do tipo Picard-Fuchs se possuir um conjunto fundamental de soluções que são integrais abelianas.

\subsection{Estudo do sistema $P_{23}$}

Llibre e Oliveira [35, 36] e Artés, Llibre e Vulpe [5] obtiveram uma classificação completa dos sistemas quadráticos com integral primeira racional de grau três. Nessa classificação foram obtidas trinta e uma classes de sistemas das quais sete apresentam centros. Propomos o estudo das bifurcações dos ciclos limites de um dos sistemas encontrados cuja classe foi denominada $P_{23}$.

Consideremos sistemas cuja forma canônica seja dada por

$$
\left\{\begin{array}{l}
\dot{x}=x y \\
\dot{y}=b+e x+\ell x^{2}+\frac{3}{2} y^{2}
\end{array}\right.
$$

onde $(b, e, \ell)$ é tal que $b, \ell \in\{-1,0,1\}, e \in \mathbb{R}^{+} \cup\{0\}$. Uma integral primeira racional de grau 3 é dada por

$$
H(x, y)=\frac{2 b+3 e x+6 \ell x^{2}+3 y^{2}}{x^{3}}
$$

Para a classe de sistemas $P_{23}$, temos $(b, e, \ell)=(1,1,0)$. Logo,

$$
\left\{\begin{array}{l}
\dot{x}=x y \\
\dot{y}=1+x+\frac{3}{2} y^{2}
\end{array}\right.
$$


$\mathrm{e}$

$$
H(x, y)=\frac{2+3 x+3 y^{2}}{x^{3}}
$$

O ponto $\left(x_{0}, y_{0}\right)=(-1,0)$ é uma singularidade de (1.6.1) do tipo centro. A Figura 1.5 ilustra o retrato de fase do sistema (1.6.1) no disco de Poincaré.

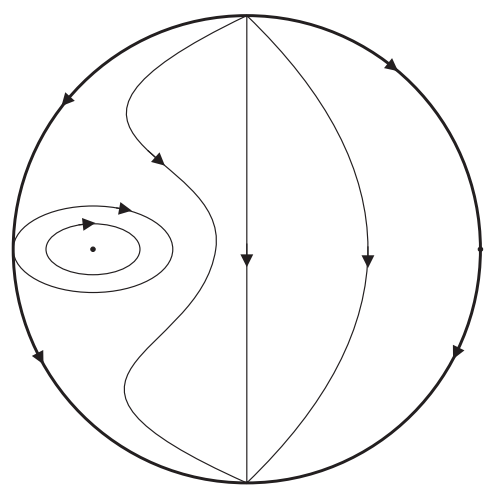

Figura 1.5: Retrato de fase do sistema (1.6.1) no disco de Poincaré.

Notemos que:

$$
\frac{\partial H}{\partial x}(x, y)=-\frac{6}{x^{4}}\left(1+x+\frac{3}{2} y^{2}\right)=-\frac{6}{x^{4}} \dot{y}
$$

e

$$
\frac{\partial H}{\partial y}(x, y)=\frac{6}{x^{4}} x y=\frac{6}{x^{4}} \dot{x} .
$$

Tomando $M(x, y)=\frac{x^{4}}{6}$, o sistema (1.6.1) escreve-se na forma

$$
\left\{\begin{array}{l}
\dot{x}=\frac{1}{M(x, y)} \cdot \frac{\partial H}{\partial y}(x, y), \\
\dot{y}=-\frac{1}{M(x, y)} \cdot \frac{\partial H}{\partial x}(x, y) .
\end{array}\right.
$$

A função $M(x, y)$ é um fator integrante. Além disso, observemos que $M$ é um fator comum aos polinômios que definem o sistema. Ao multiplicarmos cada um desses polinômios pelo inverso de $M$, eles se tornam coprimos e, assim, garantimos a não existência de uma reta de singularidades.

Consideremos, agora, uma perturbação do sistema (1.6.3). Estamos interessados somente em perturbações polinomiais quadráticas. 
Seja o sistema perturbado

$$
\left\{\begin{array}{l}
\dot{x}=\frac{1}{M(x, y)} \cdot\left(\frac{\partial H}{\partial y}(x, y)+\varepsilon f(x, y)\right) \\
\dot{y}=-\frac{1}{M(x, y)} \cdot\left(\frac{\partial H}{\partial x}(x, y)+\varepsilon g(x, y)\right)
\end{array}\right.
$$

onde $f(x, y)=\sum_{i+j=0}^{2} a_{i j} x^{i} y^{j}$ e $g(x, y)=\sum_{i+j=0}^{2} b_{i j} x^{i} y^{j}$ são polinômios quadráticos em $x$ e $y$, $a_{i j}, b_{i j} \in \mathbb{R}, i, j \in\{0,1,2\}$, e $\varepsilon>0$ é suficientemente pequeno.

Seja $C=\left\{(x, y) \in \mathbb{R}^{2} ; H(x, y)=h, h \in \mathbb{R}\right\}$ o conjunto das curvas de nível de $H$.

Lema 1.6.1. As curvas de nível $\left\{\gamma_{h}\right\}$, para $0<h<1$, é a família contínua de ovais contida em $C$.

Demonstração: Notemos que

$$
H(-1,0)=\frac{2+3(-1)}{(-1)^{3}}=1
$$

Por outro lado,

$$
\lim _{x \rightarrow-1} H(x, 0)=\lim _{x \rightarrow-1} \frac{2+3 x}{x^{3}}=1
$$

e

$$
\lim _{x \rightarrow-\infty} H(x, 0)=\lim _{x \rightarrow-\infty} \frac{2+3 x}{x^{3}}=\lim _{x \rightarrow-\infty}\left(\frac{2}{x^{3}}+\frac{3}{x^{2}}\right)=0 .
$$

Portanto, a família contínua de ovais é dada por $\gamma_{h}=\left\{(x, y) \in \mathbb{R}^{2} ; H(x, y)=h\right.$, $h \in(0,1)\} \subset C$.

Consideremos a 1-forma racional $\omega=\frac{f(x, y)}{M(x, y)} d y-\frac{g(x, y)}{M(x, y)} d x$, e a integral abeliana

$$
I(h)=\oint_{\gamma_{h}} \frac{f(x, y)}{M(x, y)} d y-\frac{g(x, y)}{M(x, y)} d x
$$

Dessa forma,

$$
I(h)=6 \oint_{\gamma_{h}}\left(\sum_{i+j=0}^{2} a_{i j} x^{i-4} y^{j} d y-\sum_{i+j=0}^{2} b_{i j} x^{i-4} y^{j} d x\right) .
$$


Denotemos

$$
I_{i j}(h)=\oint_{\gamma_{h}} x^{i-4} y^{j} d x \quad \text { e } \quad J_{i}(h)=I_{i 1}(h)
$$

Como $H(x,-y)=H(x, y)$, temos $I_{i, 2 k}(h)=0, k=0,1,2, \ldots$

Por outro lado, consideremos $I_{i j}^{y}(h)=\oint_{\gamma_{h}} x^{i-4} y^{j} d y$, e fazendo $\tilde{y}=y^{j+1}$, temos $d \tilde{y}=(j+1) y^{j} d y$.

Logo,

$$
I_{i j}^{y}(h)=\frac{1}{j+1} \oint_{\gamma_{h}} x^{i-4} d \tilde{y}
$$

Sendo $K_{h}$ a região compacta delimitada por $\gamma_{h}$, segue do Teorema de Green que

$$
\begin{aligned}
I_{i j}^{y}(h) & =\frac{1}{j+1} \iint_{K_{h}} \frac{\partial}{\partial x}\left(x^{i-4}\right) d x d \tilde{y}=\frac{i-4}{j+1} \iint_{K_{h}} x^{i-5} d x d \tilde{y}= \\
& =-\frac{i-4}{j+1} \oint_{\gamma_{h}} x^{i-5} \tilde{y} d x=-\frac{i-4}{j+1} \oint_{\gamma_{h}} x^{i-5} y^{j+1} d x= \\
& =-\frac{i-4}{j+1} I_{i-1, j+1}(h) .
\end{aligned}
$$

Observemos que, do sistema (1.6.1), obtemos

$$
x^{-3} y \frac{d y}{d x}=\frac{1}{x^{4}}+\frac{1}{x^{3}}+\frac{3 y^{2}}{2 x^{4}}
$$

Multiplicando (1.6.7) por $x^{i} y^{j-2}$ e integrando ao longo de $\gamma_{h}$, temos

$$
\begin{gathered}
\oint_{\gamma_{h}} x^{i-3} y^{j-1} d y-\oint_{\gamma_{h}}\left(x^{i-4} y^{j-2}+x^{i-3} y^{j-2}+\frac{3}{2} x^{i-4} y^{j}\right) d x=0 \Rightarrow \\
\Rightarrow-\frac{i-3}{j} I_{i j}-I_{i, j-2}-I_{i+1, j-2}-\frac{3}{2} I_{i j}=0 \Rightarrow \\
\Rightarrow-\frac{2 i+3 j-6}{2 j} I_{i j}=I_{i, j-2}+I_{i+1, j-2} \Rightarrow \\
\Rightarrow I_{i j}=-\frac{2 j}{2 i+3 j-6}\left(I_{i, j-2}+I_{i+1, j-2}\right) .
\end{gathered}
$$


Considerando (1.6.5), segue que

$$
\begin{aligned}
I(h)= & 6\left(a_{00} I_{00}^{y}(h)+a_{01} I_{01}^{y}(h)+a_{10} I_{10}^{y}(h)+a_{11} I_{11}^{y}(h)+a_{20} I_{20}^{y}(h)+a_{02} I_{02}^{y}(h)-\right. \\
& \left.-b_{00} I_{00}(h)-b_{01} I_{01}(h)-b_{10} I_{10}(h)-b_{11} I_{11}(h)-b_{20} I_{20}(h)-b_{02} I_{02}(h)\right)= \\
\stackrel{(1.6 .6)}{=} 6 & \left(4 a_{00} I_{-11}(h)+2 a_{01} I_{-12}(h)+3 a_{10} I_{01}(h)+\frac{3}{2} a_{11} I_{02}(h)+2 a_{20} I_{11}(h)+\right. \\
& \left.+\frac{4}{3} a_{02} I_{-13}(h)-b_{01} I_{01}(h)-b_{11} I_{11}(h)\right)= \\
= & 6\left(4 a_{00} J_{-1}(h)+3 a_{10} J_{0}(h)+2 a_{20} J_{1}(h)+\frac{4}{3} a_{02} I_{-13}(h)-b_{01} J_{0}(h)-b_{11} J_{1}(h)\right) .
\end{aligned}
$$

Por (1.6.8),

$$
I_{-13}(h)=-\frac{6}{-2+9-6}\left(I_{-11}(h)+I_{01}(h)\right)=-6 J_{-1}(h)-6 J_{0}(h) .
$$

Logo,

$$
I(h)=\left(24 a_{00}-48 a_{02}\right) J_{-1}(h)+\left(18 a_{10}-48 a_{02}+-6 b_{01}\right) J_{0}(h)+\left(12 a_{20}-6 b_{11}\right) J_{1}(h),
$$

isto é, a integral abeliana $I(h)$ é expressa como uma combinação linear das três integrais abelianas $J_{-1}(h), J_{0}(h)$ e $J_{1}(h)$.

Ao iniciarmos o estudo do sistema $P_{23}$ nosso principal objetivo era de aplicar a teoria das integrais abelianas para encontrar um limitante superior para o número de ciclos limites do sistema perturbado. Porém, enfrentamos alguns obstáculos durante o nosso trabalho.

Nosso primeiro passo foi calcular as integrais abelianas $J_{-1}(h), J_{0}(h)$ e $J_{1}(h)$. Utilizamos como ferramenta auxiliar para os cálculos os manipuladores algébrico-numéricos Maple e Mathematica. Inicialmente, entramos com os dados nos programas para calcular a primitiva da função $J_{0}(h)$ e, em seguida, utilizando o Teorema Fundamental do Cálculo, avaliamos essa expressão nos extremos do intervalo de integração (que são raízes da função $y=y(x, h)$ provinda da expressão de $H(x, y))$.

O Maple dava-nos como resposta o valor zero. O Mathematica apresentou dificuldade na resolução dos cálculos, dando como resposta ao comando a seguinte expressão: 


\section{ComplexInfinity.}

Após trabalharmos com as três integrais $J_{-1}(h), J_{0}(h)$ e $J_{1}(h)$ e verificarmos as mesmas dificuldades acima, passamos a acreditar (devido à resposta do Maple) que o sistema $P_{23}$ poderia tratar-se de um caso degenerado, isto é, que a integral abeliana $I(h)$ fosse identicamente nula para $h \in(0,1)$ e dessa forma, de acordo com a Observação 1.4.11, o limitante superior que fosse obtido seria menor do que o esperado para o número de zeros de $I(h)$ e teríamos de considerar aproximações de ordem maior para a função sucessão.

Devido a esta incerteza, apresentamos o problema ao professor Joan C. Artés (UAB/ Barcelona), que contribuiu de forma significativa e positiva para o andamento do nosso estudo. Constatamos que os dois programas apresentam dificuldades algébricas na resolução dos cálculos desejados. Chegamos a esta conclusão após calcularmos as integrais numericamente, onde verificamos que a integral abeliana $I(h)$, para um dado valor de $h \in(0,1)$, era distinta de zero e, portanto, não degenerada.

Buscando compreender melhor as dificuldades apresentadas pelo Maple e pelo Mathematica para realizar os cálculos algébricos, Artés nos mostrou como substituir os cálculos algébricos por cálculos numéricos e, através deles, obter os gráficos das funções $J_{-1}(h), J_{0}(h)$ e $J_{1}(h)$.

Observando o gráfico de $J_{0}(h)$ verificamos o que levou à dificuldade algébrica dos programas, como ilustra a Figura 1.6.

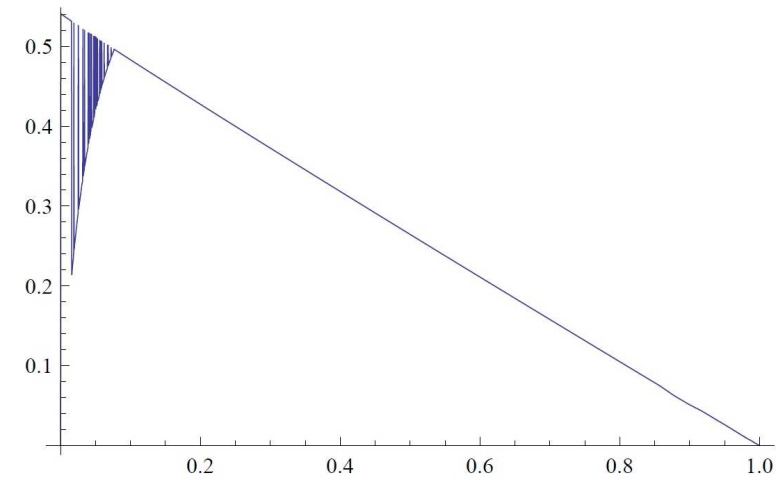

Figura 1.6: Gráfico da função $J_{0}(h)$.

Próximo a $h=0$, o gráfico da função $J_{0}(h)$ oscila, enquanto que para valores de $h$ entre 0,2 e 1 , a função $J_{0}(h)$ comporta-se aproximadamente como uma reta. Dessa forma, não conseguimos encontrar uma expressão algébrica que defina essa função. Logo, não 
foi possível obter uma estimativa para o número de ciclos limites bifurcados por meio do cálculo das integrais $J_{-1}(h), J_{0}(h)$ e $J_{1}(h)$.

Outra tentativa na busca pelo número de ciclos limites que bifurcam do centro observado em $P_{23}$ foi feita seguindo passos semelhantes aos descritos na Seção 1.5.1 para o sistema de Bogdanov-Takens. Nesse caso, a barreira a ser transposta é o estudo da monotonicidade da razão entre duas integrais abelianas, isto é, a prova de um resultado análogo ao Teorema 1.5.3 para o nosso sistema.

Durante a redação final desta dissertação, no mês de janeiro, deparamo-nos com o trabalho de Li e Llibre [31], onde um sistema semelhante ao $P_{23}$ é considerado através do método envolvendo a equação de Picard-Fuchs. Pretendemos dar continuidade a nossa investigação seguindo as ideias deste trabalho. 


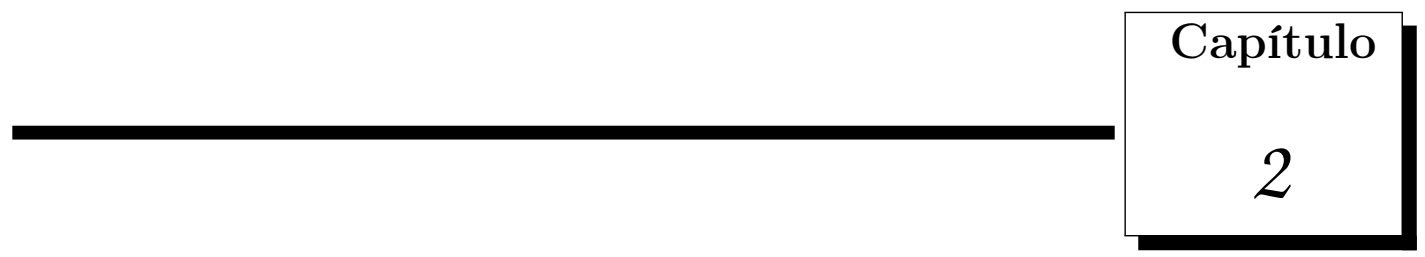

\section{A teoria do averaging}

Neste capítulo apresentamos o método conhecido como averaging. O método do averaging investiga quão próximas estão as soluções de um sistema diferencial não autônomo das soluções do sistema diferencial autônomo obtido dele. Neste segundo capítulo da dissertação apresentamos duas provas do teorema do averaging de primeira ordem para o caso analítico. Discutimos como reescrever um dado sistema diferencial na forma padrão, necessária para o uso do averaging e, em seguida, discutimos sobre a necessidade do sistema estar na forma padrão para a aplicação do método. Ainda neste capítulo, apresentamos o averaging de ordem superior e o averaging topológico, apresentado por Buică e Llibre em [9]. Em todas as discussões acima exemplos são apresentados.

Para este capítulo, dada $f$ uma função vetorial suave na variável $\varepsilon$, com $\varepsilon$ próximo a zero, escrevemos o $k$-ésimo polinômio de Taylor de $f$, ou seu $k$-jato, como

$$
\mathrm{J}_{\varepsilon}^{k} f=f^{0}+\varepsilon f^{1}+\cdots+\varepsilon^{k} f^{k}
$$

onde $f^{j}=\frac{f^{(j)}(0)}{j !}$ é o coeficiente de Taylor. A série de Taylor de $f$ de grau $k$ com resto é escrita como

$$
f(\varepsilon)=f^{0}(\varepsilon)+\varepsilon f^{1}(\varepsilon)+\cdots+\varepsilon^{k} f^{k}(\varepsilon)+\varepsilon^{k+1} f^{[k+1]}(\varepsilon) .
$$

Um índice sobrescrito denota o coeficiente de Taylor, enquanto que um índice sobrescrito 
entre colchetes denota o resto. Essa notação é facilmente estendida a funções com variáveis adicionais. Por exemplo, um campo vetorial $f$ que depende do tempo pode ser expandido como

$$
f(x, t, \varepsilon)=f^{0}(x, t)+\varepsilon f^{1}(x, t)+\cdots+\varepsilon^{k} f^{k}(x, t)+\varepsilon^{k+1} f^{[k+1]}(x, t, \varepsilon) .
$$

Nessa notação, é sempre verdade que o 0-jato de $f$ é

$$
f(x, t, \varepsilon)=f^{0}(x, t),
$$

e se $f^{0}(x, t)$ for identicamente nula (como ocorre frequentemente em problemas de averaging), então

$$
f(x, t, \varepsilon)=\varepsilon f^{[1]}(x, t, \varepsilon)
$$

\subsection{Preliminares}

\subsubsection{Teorema de existência e unicidade de soluções}

As funções vetoriais $f(x, t, \varepsilon)$ consideradas nesta dissertação têm certas propriedades com respeito às variáveis $x$ e $t$ e o parâmetro $\varepsilon$. Com respeito à variável espacial $x$, a função $f$ sempre satisfaz a condição de Lipschitz.

Observação 2.1.1. Adotemos a seguinte notação: $G=D \times\left[t_{0}, t_{0}+T\right] \times\left(0, \varepsilon_{0}\right]$.

Definição 2.1.2. A função vetorial $f: G \rightarrow \mathbb{R}^{n}$ satisfaz a condição de Lipschitz em $x$ com constante de Lipschitz $\lambda_{f}$ se

$$
\left\|f\left(x_{1}, t, \varepsilon\right)-f\left(x_{2}, t, \varepsilon\right)\right\| \leq \lambda_{f}\left\|x_{1}-x_{2}\right\| .
$$

Se f for periódica de período $T$, a condição de Lipschitz valerá para todo t.

É bem conhecido que se $f$ for de classe $\mathcal{C}^{1}$ em um subconjunto aberto $U$ em $\mathbb{R}^{n}$ e $D$ um subconjunto de $U$ com fecho $\bar{D}$ compacto e convexo, então $f$ satisfaz a condição de Lipschitz em $\bar{D}$ com $\lambda_{f}=\max \{\|\mathrm{D} f(x)\| ; x \in \bar{D}\}$. O próximo lema mostra que a convexidade não é necessária. 
Lema 2.1.3. [41] Suponhamos que $f$ seja de classe $\mathcal{C}^{1}$ em $U$, como anteriormente, e $\bar{D}$ compacto (mas não necessariamente convexo). Então, $f$ ainda é Lipschitz em $\bar{D}$.

Estamos agora aptos para formular o bem conhecido Teorema Local de Existência e Unicidade para Problemas de Valores Iniciais.

Teorema 2.1.4 (Existência e Unicidade). [41] Consideremos a equação diferencial

$$
\dot{x}=f(x, t, \varepsilon) .
$$

Estamos interessados em soluções $x$ dessa equação com valor inicial $x\left(t_{0}\right)=a$. Sejam $D=\left\{x \in \mathbb{R}^{n} ;\|x-a\|<d\right\}, G$ como na Observação 2.1.1 e $f: G \rightarrow \mathbb{R}^{n}$. Assumamos que

1. $f$ é contínua em $G$;

2. $f$ satisfaz a condição de Lipschitz como na Definição 2.1.2.

Então, o problema de valor inicial tem uma única solução $x$ que existe para $t_{0} \leq t \leq t_{0}+\inf \left(T, \frac{d}{M}\right)$, onde $M=\sup _{G}\|f\|=\|f\|_{\text {sup }}=\sup _{x \in D, 0 \leq t \leq T, 0 \leq \varepsilon \leq \varepsilon_{0}}\|f(x, t, \varepsilon)\|$.

Observação 2.1.5. Diferentes enunciados para o Teorema de Existência e Unicidade são encontrados na literatura. No entanto, optamos pela forma como foi enunciado acima em função dos resultados que seguem.

Notemos que o Teorema 2.1.4 garante a existência de uma solução em um intervalo de tempo que depende explicitamente da norma de $f$. Hipóteses adicionais permite-nos provar teoremas de continuação, ou seja, com essas suposições podemos obter a existência para intervalos maiores, ou até mesmo para todo o tempo. Veja [23].

\subsubsection{Função ordem, símbolos de Landau e aproximação assin- tótica}

Nesta seção tratamos de alguns conceitos básicos sobre aproximação assintótica. Como destaca [41], técnicas de aproximação são úteis no estudo de problemas onde temos funções 
definidas por integrais, ou como solução de uma equação diferencial ordinária, como acontece neste capítulo.

Estamos interessados em problemas de perturbação do seguinte tipo

$$
\dot{x}=f(x, t, \varepsilon), x\left(t_{0}\right)=x_{0}
$$

onde $x, x_{0} \in \mathbb{R}^{n}, t \in\left[t_{0}, \infty\right)$ e $\varepsilon \in\left(0, \varepsilon_{0}\right]$, com $\varepsilon_{0}$ um parâmetro positivo pequeno. Se o campo vetorial $f$ for suficientemente suave em uma vizinhança de $\left(x_{0}, t_{0}\right) \in \mathbb{R}^{n} \times \mathbb{R}$, o problema de valor inicial (2.1.1) terá uma única solução $x_{\varepsilon}(t)$ para pequenos valores de $\varepsilon$ fixados.

Alguns problemas que surgem nesse processo de aproximação podem ser ilustrados pelos seguintes exemplos. Consideremos a equação de primeira ordem com valor inicial

$$
\dot{x}=x+\varepsilon, x_{\varepsilon}(0)=1 .
$$

A solução é $x_{\varepsilon}(t)=(1+\varepsilon) e^{t}-\varepsilon$. Escrevendo essa expressão com respeito a $\varepsilon$, obtemos

$$
x_{\varepsilon}(t)=e^{t}+\varepsilon\left(e^{t}-1\right) .
$$

Esse resultado sugere que a função $e^{t}$ seja uma aproximação, em algum sentido, para $x_{\varepsilon}(t)$, se $t$ não for muito grande. Na definição do conceito de aproximação certamente é necessário considerar o domínio de validade de tal aproximação.

O segundo exemplo mostra que a solução nem sempre depende do parâmetro $\varepsilon$ de forma suave. Seja

$$
\dot{x}=-\frac{\varepsilon x}{\varepsilon+t}, x_{\varepsilon}(0)=1
$$

A solução é dada por

$$
x_{\varepsilon}(t)=\left(\frac{\varepsilon}{\varepsilon+t}\right)^{\varepsilon} .
$$

Para caracterizar o comportamento da solução com relação a $\varepsilon$ para $t \geq 0$ devemos dividir 
$\mathbb{R}^{+}$em dois diferentes domínios. Por exemplo, é possível escrever

$$
x_{\varepsilon}(t)=1+\varepsilon \ln \varepsilon-\varepsilon \ln t+\mathcal{O}\left(\frac{\varepsilon}{t}\right)
$$

onde $\mathcal{O}\left(\frac{\varepsilon}{t}\right)$ é pequeno comparado aos outros termos. Essa expansão é possível quando $t$ estiver contido em um intervalo $I_{\varepsilon}$ dependente de $\varepsilon \operatorname{com} \frac{\varepsilon}{t}$ pequeno. Tal expansão não satisfaz a condição inicial.

A partir de agora, introduzimos alguns conceitos que nos ajudarão a discutir os problemas acima citados.

Definição 2.1.6. Uma função $\delta(\varepsilon)$ será chamada de função ordem se $\delta(\varepsilon)$ for contínua e positiva em $\left(0, \varepsilon_{0}\right]$ e se $\lim _{\varepsilon \rightarrow 0} \delta(\varepsilon)$ existir.

Para comparar funções ordem usamos os simbolos de Landau, que também são conhecidos como $\mathcal{O}$-grande e $o$-pequeno.

Definição 2.1.7. Seja $\varphi(t, \varepsilon)$ uma função real (ou vetorial) definida para $\varepsilon>0$ (ou $\varepsilon \geq 0$ ) e para $t \in I_{\varepsilon}$. (A expressão "para $\varepsilon \rightarrow 0$ " significa dizer que existe um $\varepsilon_{0}>0$ tal que a afirmação valha para todo $\left.\varepsilon \in\left(0, \varepsilon_{0}\right]\right)$. Definimos os símbolos de Landau $\mathcal{O}(\cdot)$ e o $(\cdot)$ como se segue.

1. Diremos que $\varphi(t, \varepsilon)=\mathcal{O}(\delta(\varepsilon))$ para $\varepsilon \rightarrow 0$ se existirem constantes $\varepsilon_{0}>0$ e $k>0$ tais que

$$
\|\varphi(t, \varepsilon)\| \leq k|\delta(\varepsilon)|
$$

para todo $t \in I_{\varepsilon}$ e para $0<\varepsilon<\varepsilon_{0}$.

2. Diremos que $\varphi(t, \varepsilon)=o(\delta(\varepsilon))$ para $\varepsilon \rightarrow 0$ se

$$
\lim _{\varepsilon \rightarrow 0} \frac{\|\varphi(t, \varepsilon)\|}{\delta(\varepsilon)}=0
$$

uniformemente para $t \in I_{\varepsilon}$. (Isto é, para todo $\alpha>0$, existe $\beta>0$ tal que $\frac{\|\varphi(t, \varepsilon)\|}{\delta(\varepsilon)}<\alpha$ se $t \in I_{\varepsilon}$ e $\left.0<\varepsilon<\beta\right)$. 
3. Diremos que $\delta_{1}(\varepsilon)=o\left(\delta_{2}(\varepsilon)\right)$ para $\varepsilon \rightarrow 0$ se

$$
\lim _{\varepsilon \rightarrow 0} \frac{\delta_{1}(\varepsilon)}{\delta_{2}(\varepsilon)}=0
$$

Exemplo 2.1.8. Os exemplos a seguir ilustram o uso dos símbolos de Landau.

1. $\varepsilon^{n}=o\left(\varepsilon^{m}\right)$ para $\varepsilon \rightarrow 0$, se $n>m$. De fato,

$$
\lim _{\varepsilon \rightarrow 0} \frac{\varepsilon^{n}}{\varepsilon^{m}}=\lim _{\varepsilon \rightarrow 0} \varepsilon^{n-m}=0 .
$$

2. $\varepsilon \operatorname{sen} \frac{1}{\varepsilon}=\mathcal{O}(\varepsilon)$ para $\varepsilon \rightarrow 0$. De fato,

$$
\left|\varepsilon \operatorname{sen} \frac{1}{\varepsilon}\right|=|\varepsilon|\left|\operatorname{sen} \frac{1}{\varepsilon}\right| \leq|\varepsilon| .1=|\varepsilon| .
$$

Tome $k=1$.

3. $\varepsilon^{2} \ln \varepsilon=o\left(\varepsilon^{2} \ln ^{2} \varepsilon\right)$ para $\varepsilon \rightarrow 0$. De fato,

$$
\lim _{\varepsilon \rightarrow 0} \frac{\varepsilon^{2} \ln \varepsilon}{\varepsilon^{2} \ln ^{2} \varepsilon}=\lim _{\varepsilon \rightarrow 0} \frac{1}{\ln \varepsilon}=0 .
$$

4. $e^{-\frac{1}{\varepsilon}}=o\left(\varepsilon^{n}\right)$, para todo $n \in \mathbb{N}$ e para $\varepsilon \rightarrow 0$. De fato,

$$
\lim _{\varepsilon \rightarrow 0} \frac{e^{-\frac{1}{\varepsilon}}}{\varepsilon^{n}}=\lim _{\varepsilon \rightarrow 0} \frac{e^{-\frac{1}{\varepsilon}}}{e^{n \ln \varepsilon}}=\lim _{\varepsilon \rightarrow 0} e^{-\frac{1}{\varepsilon}-n \ln \varepsilon}=0 .
$$

Notemos que $\delta_{1}(\varepsilon)=o\left(\delta_{2}(\varepsilon)\right)$ implica $\delta_{1}(\varepsilon)=\mathcal{O}\left(\delta_{2}(\varepsilon)\right)$. Por exemplo, $\varepsilon^{2}=o(\varepsilon)$ e $\varepsilon^{2}=\mathcal{O}(\varepsilon)$ quando $\varepsilon \rightarrow 0$. Introduzimos, agora, a noção de uma estimativa mais fina para funções ordem.

Definição 2.1.9. Diremos que $\delta_{1}(\varepsilon)=\mathcal{O}_{s}\left(\delta_{2}(\varepsilon)\right)$ para $\varepsilon \rightarrow 0$ se $\delta_{1}(\varepsilon)=\mathcal{O}\left(\delta_{2}(\varepsilon)\right)$ e $\delta_{1}(\varepsilon) \neq o\left(\delta_{2}(\varepsilon)\right)$ para $\varepsilon \rightarrow 0$.

Exemplo 2.1.10. Temos $\varepsilon \operatorname{sen}\left(\frac{1}{\varepsilon}\right)=\mathcal{O}_{s}(\varepsilon)$ e $\varepsilon \ln \varepsilon=\mathcal{O}_{s}\left(2 \varepsilon \ln \varepsilon+\varepsilon^{3}\right)$. 
A variável real $t$ usada no problema de valor inicial (2.1.1) é chamada de tempo. Devemos também ter variáveis tempo-escala da forma $\tau=\delta(\varepsilon) t \operatorname{com} \delta(\varepsilon)=\mathcal{O}(1)$. Podemos, agora, estimar a ordem de grandeza de funções $\varphi(t, \varepsilon)$, também escritas como $\varphi_{\varepsilon}(t)$, definidas em um intervalo $I_{\varepsilon}, \varepsilon \in\left(0, \varepsilon_{0}\right]$.

Definição 2.1.11. Suponhamos que $\varphi_{\varepsilon}(t): I_{\varepsilon} \rightarrow \mathbb{R}^{n}$ para $0<\varepsilon<\varepsilon_{0}$. Sejam $\|\cdot\| a$ métrica Euclidiana em $\mathbb{R}^{n}$, e $|\cdot|$ definida por

$$
\left|\varphi_{\varepsilon}(t)\right|=\sup \left\{\left\|\varphi_{\varepsilon}(t)\right\| ; t \in I_{\varepsilon}\right\}
$$

(Notemos que essa norma depende de $\varepsilon$ e poderia ter sido escrita mais precisamente como $\left.|\cdot|_{\varepsilon}.\right)$ Seja $\delta$ uma função ordem. Então,

1. $\varphi_{\varepsilon}=\mathcal{O}(\delta(\varepsilon))$ em $I_{\varepsilon}$ se $\left|\varphi_{\varepsilon}\right|=\mathcal{O}(\delta(\varepsilon))$ para $\varepsilon \rightarrow 0$;

2. $\varphi_{\varepsilon}=o(\delta(\varepsilon))$ em $I_{\varepsilon}$ se $\lim _{\varepsilon \rightarrow 0} \frac{\left|\varphi_{\varepsilon}\right|}{\delta(\varepsilon)}=0$;

3. $\varphi_{\varepsilon}=\mathcal{O}_{s}(\delta(\varepsilon))$ em $I_{\varepsilon}$ se $\varphi_{\varepsilon}=\mathcal{O}(\delta(\varepsilon))$ e $\varphi_{\varepsilon} \neq o(\delta(\varepsilon))$.

É comum dizer que as estimativas definidas nesse sentido são uniformes ou uniformemente válidas em $I_{\varepsilon}$, devido ao uso de $|\cdot|$, que faz a estimativa ficar independente de $t$.

A Definição 2.1.11 pode ser adaptada e enunciada para variáveis espaciais.

Exemplo 2.1.12. Desejamos estimar a ordem de grandeza do erro quando aproximamos $\operatorname{sen}(t+\varepsilon t)$ por sen $t$ no intervalo $I_{\varepsilon}$. Se $I_{\varepsilon}=[0,2 \pi]$, teremos para a diferença das duas funções, usando série de Taylor, que

$$
\operatorname{sen}(t+\varepsilon t)=\operatorname{sen} t+r, \text { onde } \lim _{\varepsilon \rightarrow 0} \frac{r}{\varepsilon}=0 .
$$

Dessa forma,

$$
\sup _{t \in[0,2 \pi]}|\operatorname{sen}(t+\varepsilon t)-\operatorname{sen} t|=\sup _{t \in[0,2 \pi]}|r| \Rightarrow \frac{\sup _{t \in[0,2 \pi]}|\operatorname{sen}(t+\varepsilon t)-\operatorname{sen} t|}{\varepsilon}=\frac{\sup _{t \in[0,2 \pi]}|r|}{\varepsilon} \Rightarrow
$$




$$
\Rightarrow \frac{\sup _{t \in[0,2 \pi]}|\operatorname{sen}(t+\varepsilon t)-\operatorname{sen} t|}{\varepsilon}=\sup _{t \in[0,2 \pi]}\left|\frac{r}{\varepsilon}\right| \stackrel{\varepsilon \rightarrow 0}{\Rightarrow} \frac{\sup _{t \in[0,2 \pi]}|\operatorname{sen}(t+\varepsilon t)-\operatorname{sen} t|}{\varepsilon} \rightarrow 0
$$

quando $\varepsilon \rightarrow 0$. Logo, $\sup _{t \in[0,2 \pi]}|\operatorname{sen}(t+\varepsilon t)-\operatorname{sen} t|=o(\varepsilon)$ e, portanto,

$$
\sup _{t \in[0,2 \pi]}|\operatorname{sen}(t+\varepsilon t)-\operatorname{sen} t|=\mathcal{O}(\varepsilon) \text {. }
$$

Observação 2.1.13. Em muitos problemas, a fronteira de $I_{\varepsilon}$ depende de $\varepsilon$ de tal forma que o tempo não seja uniformemente limitado (o intervalo se torna ilimitado quando $\varepsilon$ tender a 0$)$. Por exemplo, se comparássemos $\operatorname{sen}(t+\varepsilon t)$ com sen $t$ no intervalo $I_{\varepsilon}=\left[0, \frac{2 \pi}{\varepsilon}\right]$, obteríamos, na norma do supremo, que

$$
\operatorname{sen}(t+\varepsilon t)-\operatorname{sen} t=\mathcal{O}_{s}(1)
$$

Suponhamos que $\delta(\varepsilon)=o(1)$ e desejamos estimar $\varphi_{\varepsilon}$ em $I_{\varepsilon}=\left[0, \frac{L}{\delta(\varepsilon)}\right]$ com $L$ uma constante independente de $\varepsilon$. Tal estimativa será dada como

$$
\varphi_{\varepsilon}=\mathcal{O}(\delta(\varepsilon)), \text { quando } \varepsilon \rightarrow 0 \text { em } I_{\varepsilon}
$$

ou ainda como

$$
\varphi_{\varepsilon}(t)=\mathcal{O}(\delta(\varepsilon)) \text {, quando } \varepsilon \rightarrow 0 \text { em } I_{\varepsilon}
$$

A primeira maneira, sem a variável $t$, é preferível, mas é difícil de ser usada em um exemplo como

$$
\operatorname{sen}(t+\varepsilon t)-\operatorname{sen} t=\mathcal{O}(1)
$$

quando $\varepsilon \rightarrow 0$ em $I_{\varepsilon}$. Expressaremos essas estimativas como se segue.

Definição 2.1.14. Diremos que $\varphi_{\varepsilon}(t)=\mathcal{O}(\delta(\varepsilon))$ quando $\varepsilon \rightarrow 0$ no tempo escala $\delta(\varepsilon)^{-1}$ se a estimativa for válida para $0 \leq \delta(\varepsilon) t \leq L$, com $L$ uma constante independente de $\varepsilon$.

Uma definição análoga pode ser dada para estimativas de ordem $o(\delta(\varepsilon))$. Já que 
sabemos estimar funções em termos de funções ordem, podemos definir o conceito de aproximação assintótica.

Definição 2.1.15. Definimos aproximações assintóticas como se segue.

1. $\psi_{\varepsilon}(t)$ é uma aproximação assintótica de $\varphi_{\varepsilon}(t)$ no intervalo $I_{\varepsilon}$ se

$$
\varphi_{\varepsilon}(t)-\psi_{\varepsilon}(t)=o(1)
$$

quando $\varepsilon \rightarrow 0$, uniformemente para $t \in I_{\varepsilon}$. Ou reescrito em termos de tempo escala:

2. $\psi_{\varepsilon}(t)$ é uma aproximação assintótica de $\varphi_{\varepsilon}(t)$ no tempo escala $\delta(\varepsilon)^{-1}$ se

$$
\varphi_{\varepsilon}-\psi_{\varepsilon}=o(1)
$$

quando $\varepsilon \rightarrow 0$ no tempo escala $\delta(\varepsilon)^{-1}$.

Em geral, é possível obter séries (ou expansões) como aproximações assintóticas em algum intervalo $I_{\varepsilon}$. Uma série assintótica é uma expressão da forma

$$
\varphi(t, \varepsilon)=\sum_{j=1}^{\infty} \delta_{j}(\varepsilon) \varphi^{j}(t, \varepsilon)
$$

na qual $\delta_{j}(\varepsilon)$ são funções ordem $\operatorname{com} \delta_{j+1}=o\left(\delta_{j}\right)$.

Nas próximas seções tratamos do método do averaging que se resume em investigar quão próximas estão as soluções de um sistema diferencial não autônomo das soluções do sistema diferencial autônomo obtido dele.

\subsection{Averaging periódico de primeira ordem analítico}

O método do averaging é um método que visa encontrar soluções aproximadas para sistemas de equações diferenciais que tenham, ou que possam ser colocadas, na forma

$$
\dot{x}=\varepsilon f^{1}(x, t)+\varepsilon^{2} f^{[2]}(x, t, \varepsilon), \quad x(0)=a,
$$


onde $f^{1}$ e $f^{[2]}$ são $T$-periódicas em $t$, chamada de forma padrão.

Queremos comparar a solução da equação na forma padrão com a do sistema promediado, definido por

$$
\dot{z}=\varepsilon \bar{f}^{1}(z), \quad z(0)=a,
$$

onde

$$
\bar{f}^{1}(z)=\frac{1}{T} \int_{0}^{T} f^{1}(z, s) d s
$$

é a média de $f^{1}(z, t)$ sobre seu período $T$ em $t$. O sistema promediado (2.2.2) é mais simples do que (2.2.1), pois é autônomo, mas em geral permanece não linear e não é necessariamente solúvel. Por outro lado, como lembra Murdock [40], em muitas aplicações (principalmente no caso de dimensão dois) ele pode ser resolvido explicitamente utilizando funções elementares e, assim, é razoável investigar quando soluções de (2.2.2) podem ser aproximações para soluções de (2.2.1). Todavia, mesmo quando o sistema promediado não puder ser resolvido explicitamente, veremos que ainda é possível obter conclusões sobre as soluções do sistema original a partir das equações promediadas.

Introduzindo uma nova variável (ou escala de tempo) $\tau=\varepsilon t$ em (2.2.2), removemos $\varepsilon$ e obtemos o chamado sistema de orientação

$$
\frac{d w}{d \tau}=\bar{f}^{1}(w), \quad w(0)=a .
$$

Se a solução de (2.2.4) for $w(\tau)$, então a solução de (2.2.2) será

$$
z(t, \varepsilon)=w(\varepsilon t)
$$

Isto é, o tempo $t$ entra em $z(t, \varepsilon)$ somente na combinação $\varepsilon t$. Consideramos o ponto inicial $a$ fixado.

Nesta seção apresentamos duas provas de caráter analítico para o teorema básico do averaging de primeira ordem, que descreve condições suficientes para que a solução de (2.2.2) seja uma boa aproximação para a solução do sistema (2.2.1). A primeira demonstração é mais recente e mais curta, segue de uma desigualdade devida a Besjes [6] e utiliza o Lema de Gronwall. A segunda demonstração é mais antiga (e muito provavelmente mais 
natural), com raízes no trabalho de Bogoliubov e é mais longa, pelo menos se todos os detalhes forem tratados cuidadosamente. Assumimos que as equações diferenciais estejam definidas em todo $\mathbb{R}^{n}$, embora seja fácil adaptar os argumentos para subconjuntos abertos de $\mathbb{R}^{n}$.

Todos os argumentos nesta seção requerem, como um passo preliminar, a escolha de um conjunto $D$ aberto, limitado e conexo (com fecho $\bar{D}$ compacto) contendo $a$, uma constante $L>0$, e uma constante $\varepsilon_{0}>0$, tais que as soluções $x(t, \varepsilon)$ e $z(t, \varepsilon)$ de $(2.2 .1)$ e (2.2.2), respectivamente, com $0 \leq \varepsilon \leq \varepsilon_{0}$ permaneçam em $D$ para $0 \leq t \leq \frac{L}{\varepsilon}$. Vale ressaltar que existem duas formas de alcançar este objetivo.

1. Podemos escolher $D$ e $\varepsilon_{0}$ arbitrariamente e escolher $L$ em resposta a isso. Desde que as funções dos membros direitos de (2.2.1) e (2.2.2) são limitadas por uma constante vezes $\varepsilon$ (para $0 \leq \varepsilon \leq \varepsilon_{0}$ e para $x$ ou $z$ em $\bar{D}$ ), a existência de um $L$ adequado é óbvia.

2. Alternativamente, $L$ pode ser escolhido arbitrariamente e $D$ e $\varepsilon_{0}$ escolhidos adequadamente. Por exemplo, se a solução de (2.2.4) existir para $0 \leq \tau \leq L$, e se $D$ for uma vizinhança dessa solução, então existirá um $\varepsilon_{0}$ tal que as soluções de (2.2.1) e (2.2.2) permanecerão em $D$ para $0 \leq t \leq \frac{L}{\varepsilon}$, se $0 \leq \varepsilon \leq \varepsilon_{0}$.

Tudo isso é usualmente abreviado quando se diz que desde que as soluções $x$ e z movem-se a uma velocidade de $\mathcal{O}(\varepsilon)$, elas permanecem limitadas para tempo de $\mathcal{O}\left(\frac{1}{\varepsilon}\right)$.

Sabemos que um campo vetorial periódico de classe $\mathcal{C}^{1}$ satisfaz uma condição de Lipschitz em conjuntos compactos para todo tempo. Veja Lema 2.1.3.

\subsubsection{Primeira prova do teorema do averaging de primeira ordem}

O resultado que queremos demonstrar descreve uma condição suficiente para que a solução $z(t, \varepsilon)$ do sistema promediado seja uma boa aproximação para a solução $x(t, \varepsilon)$ do sistema original. Para a primeira prova do teorema do averaging de primeira ordem analítico precisaremos enunciar e provar três lemas que terão papéis fundamentais na demonstração. 
Lema 2.2.1 (Besjes). Suponhamos que $\varphi(x, s)$ seja periódica em s com período $T$, tenha média zero em s para $x$ fixado, seja limitada para todo $s$ e $x \in D$, e tenha constante de Lipschitz $\lambda_{\varphi}$ para $x \in D$. Suponhamos ainda que $x(t, \varepsilon)$ pertença a $D$ para $0 \leq \varepsilon \leq \varepsilon_{0} e$ $0 \leq t \leq \frac{L}{\varepsilon}$ e satisfaça $\dot{x}=\mathcal{O}(\varepsilon)$. Então, existe uma constante $c_{1}>0$ tal que

$$
\left\|\int_{0}^{t} \varphi(x(s, \varepsilon), s) d s\right\| \leq c_{1}
$$

para $0 \leq \varepsilon \leq \varepsilon_{0}$ e $0 \leq t \leq \frac{L}{\varepsilon}$

Demonstração: Primeiramente observemos que, se $x$ fosse constante, o resultado seria trivial, pois, como $T$ é o período de $\varphi$,

$$
\int_{0}^{T} \varphi(x(s, \varepsilon), s) d s
$$

é a média de $\varphi$ em $s$ e, portanto, seria nula. Como a integral é periódica, $c_{1}$ poderia ser tomada como seu valor máximo, isto é, sua amplitude.

No entanto, $x$ não é constante, pois varia lentamente. Dividamos o intervalo $[0, t]$ em períodos: $[0, T],[T, 2 T], \ldots,[(m-1) T, m T]$ e $[m T, t]$, que é menor do que um período. Então:

$$
\left\|\int_{0}^{t} \varphi(x(s, \varepsilon), s) d s\right\|=\sum_{i=1}^{m}\left\|\int_{(i-1) T}^{i T} \varphi(x(s, \varepsilon), s) d s\right\|+\left\|\int_{m T}^{t} \varphi(x(s, \varepsilon), s) d s\right\| .
$$

Cada integral sobre o período pode ser estimada como segue (veja os argumentos abaixo):

$$
\begin{aligned}
\left\|\int_{(i-1) T}^{i T} \varphi(x(s, \varepsilon), s) d s\right\| & \stackrel{(1)}{=}\left\|\int_{(i-1) T}^{i T}[\varphi(x(s, \varepsilon), s)-\varphi(x((i-1) T, \varepsilon), s)] d s\right\| \leq \\
& \leq \lambda_{\varphi} \int_{(i-1) T}^{i T}\|x(s, \varepsilon)-x((i-1) T, \varepsilon)\| d s \leq \\
& \stackrel{(2)}{\leq} \lambda_{\varphi} \int_{(i-1) T}^{i T} c_{2} \varepsilon d s=\lambda_{\varphi} c_{2} \varepsilon T .
\end{aligned}
$$


A igualdade (1) é válida, pois:

$$
\int_{(i-1) T}^{i T} \varphi(x((i-1) T, \varepsilon), s) d s=\int_{u_{0}}^{u_{0}+T} \varphi\left(x\left(u_{0}, \varepsilon\right), s\right) d s=0
$$

já que representa a média para $x$ fixo.

Em (2), a limitação

$$
\|x(s, \varepsilon)-x((i-1) T, \varepsilon)\| \leq c_{2} \varepsilon
$$

para algum $c_{2}$, segue do movimento lento de $x$.

A última integral sobre um período parcial é limitada por $c_{3}=\|\varphi\| T$.

Então,

$$
\left\|\int_{0}^{t} \varphi(x(s, \varepsilon), s) d s\right\| \leq m \lambda_{\varphi} c_{2} T \varepsilon+c_{3} .
$$

Mas, pela construção,

$$
m T \leq t \leq \frac{L}{\varepsilon}
$$

então

$$
m \lambda_{\varphi} c_{2} T \varepsilon+c_{3} \leq \lambda_{\varphi} c_{2} L+c_{3}
$$

Tomemos $c_{1}=\lambda_{\varphi} c_{2} L+c_{3}$.

Lema 2.2.2 (Gronwall Geral). Suponhamos que para $t_{0} \leq t \leq t_{0}+T$ tenhamos

$$
\varphi(t) \leq \alpha+\int_{t_{0}}^{t} \beta(s) \varphi(s) d s
$$

onde $\varphi$ e $\beta$ são contínuas e $\beta(t)>0$. Então,

$$
\varphi(t) \leq \alpha e^{\int_{t_{0}}^{t} \beta(s)} d s
$$

para $t_{0} \leq t \leq t_{0}+T$.

Demonstração: Seja

$$
\psi(t)=\alpha+\int_{t_{0}}^{t} \beta(s) \varphi(s) d s .
$$

Então, $\varphi(t) \leq \psi(t)$ e $\dot{\psi}(t)=\beta(t) \varphi(t)$. Assim, já que $\beta(t)>0, \dot{\psi}(t)-\beta(t) \psi(t) \leq 0$. 
Essa inequação diferencial pode ser tratada exatamente como se resolvêssemos a equação diferencial correspondente. Isto é, ela pode ser reescrita como

$$
\frac{d}{d t}\left(\psi(t) e^{-\int_{t_{0}}^{t} \beta(s) d s}\right) \leq 0
$$

e integrada de $t_{0}$ a $t$, com $\psi\left(t_{0}\right)=\alpha$, para obtermos

$$
\psi(t) e^{-\int_{t_{0}}^{t} \beta(s) d s}-\alpha \leq 0
$$

levando-nos ao resultado desejado.

Lema 2.2.3 (Gronwall Específico). Suponhamos que para $t_{0} \leq t \leq t_{0}+T$ tenhamos

$$
\varphi(t) \leq \delta_{2}\left(t-t_{0}\right)+\delta_{1} \int_{t_{0}}^{t} \varphi(s) d s+\delta_{3}
$$

com $\varphi(t)$ contínua para $t_{0} \leq t \leq t_{0}+T$ e constantes $\delta_{1}>0, \delta_{2} \geq 0$ e $\delta_{3} \geq 0$. Então,

$$
\varphi(t) \leq \frac{\delta_{2}}{\delta_{1}+\delta_{3}} e^{\delta_{1}\left(t-t_{0}\right)}-\frac{\delta_{2}}{\delta_{1}}
$$

para $t_{0} \leq t \leq t_{0}+T$

Demonstração: Este lema tem a forma do Lema 2.2.2 com $\alpha=\frac{\delta_{2}}{\delta_{1}+\delta_{3}}$ e $\beta(t)=\delta_{1}$ para todo $t$, e o resultado segue como anteriormente.

Enfim, enunciamos e provamos o teorema do averaging de primeira ordem.

Teorema 2.2.4. Suponhamos que $f^{1}$ seja Lipschitz contínua, $f^{[2]}$ seja continua, e $\varepsilon_{0}, D$ e L sejam como acima. Então, existe uma constante $c>0$ tal que

$$
\|x(t, \varepsilon)-z(t, \varepsilon)\|<c \varepsilon
$$

para $0 \leq \varepsilon \leq \varepsilon_{0}$ e $0 \leq t \leq \frac{L}{\varepsilon}$, onde $x(t, \varepsilon)$ é solução do sistema original $(2.2 .1)$ e $z(t, \varepsilon)$ é solução do sistema promediado (2.2.2). 
Demonstração: Seja

$$
E(t, \varepsilon)=x(t, \varepsilon)-z(t, \varepsilon)=x(t, \varepsilon)-w(\varepsilon t)
$$

o erro entre as soluções $x(t, \varepsilon)$ e $z(t, \varepsilon)$. Calculando $E$ a partir das equações diferenciais de $x$ e $z$ e integrando-as com respeito a $t$, obtemos

$$
E(t, \varepsilon)=\varepsilon \int_{0}^{t}\left[f^{1}(x(s, \varepsilon), s)+\varepsilon f^{[2]}(x(s, \varepsilon), s, \varepsilon)-\bar{f}^{1}(w(\varepsilon s))\right] d s
$$

Omitindo os argumentos de $x$ e $w$, o integrando pode ser reescrito como

$$
\left[f^{1}(x, s)-f^{1}(w, s)\right]+\varepsilon f^{[2]}(x, s, \varepsilon)+\left[f^{1}(w, s)-\bar{f}^{1}(w)\right]
$$

levando-nos a

$$
\begin{aligned}
\|E(t, \varepsilon)\| & \leq \varepsilon \int_{0}^{t}\left\|f^{1}(x, s)-f^{1}(w, s)\right\| d s+\varepsilon^{2}\left\|\int_{0}^{t} f^{[2]}(x, s, \varepsilon) d s\right\|+ \\
& +\varepsilon\left\|\int_{0}^{t}\left[f^{1}(w, s)-\bar{f}^{1}(w)\right] d s\right\| .
\end{aligned}
$$

Na primeira integral, usamos a constante de Lipschitz $\lambda_{f^{1}}$. Como $f^{[2]}$ é contínua e periódica, ela é limitada em $\bar{D}$ para todo tempo. Segue do Lema 2.2.1, aplicado a $\varphi=f^{1}-\bar{f}^{1}$ e $x=w(\varepsilon t)$, que a terceira integral é limitada por $c_{1} \varepsilon$, onde $c_{1}$ é uma constante. Assim, temos

$$
\|E(t, \varepsilon)\| \leq \varepsilon \lambda_{f^{1}} \int_{0}^{t}\|E(s, \varepsilon)\| d s+c_{0} \varepsilon^{2} t+c_{1} \varepsilon
$$

para $c_{0}$ e $c_{1}$ constantes adequadas.

Segue do Lema de Gronwall Específico (Lema 2.2.3) que

$$
\begin{aligned}
\|E(t, \varepsilon)\| & \leq \frac{\varepsilon^{2} c_{0}}{\varepsilon \lambda_{f^{1}}+\varepsilon c_{1}} e^{\varepsilon \lambda_{f^{1}} t}-\frac{\varepsilon^{2} c_{0}}{\varepsilon \lambda_{f^{1}}} \leq \\
& \leq \varepsilon \frac{c_{0}}{\lambda_{f^{1}}+c_{1}}\left(e^{\lambda_{f^{1}} L}-1\right),
\end{aligned}
$$


para $0 \leq \varepsilon \leq \varepsilon_{0}$ e $0 \leq t \leq \frac{L}{\varepsilon}$.

Tomando $c=\frac{c_{0}}{\lambda_{f^{1}}+c_{1}}\left(e^{\lambda_{f^{1}} L}-1\right)$, o teorema fica provado.

\subsubsection{Segunda prova do teorema do averaging de primeira ordem}

A segunda e mais tradicional demonstração do teorema do averaging de primeira ordem analítico introduz a noção de uma perturbação da identidade e também necessita de uma preparação e resultados auxiliares. Para evitar hipóteses adicionais, assumimos que todas as funções são suaves, isto é, infinitamente diferenciáveis. Uma perturbação da identidade é na verdade uma família de transformações dependendo de $\varepsilon$ que se reduz à identidade quando $\varepsilon=0$ e tem a seguinte forma:

$$
x=U(y, t, \varepsilon)=y+\varepsilon u^{[1]}(y, t, \varepsilon),
$$

onde $u^{[1]}$ é periódica em $t$ com período $T$ e $y$ é a nova variável vetorial que substituirá $x$. O objetivo é escolher $u^{[1]}$ de forma que (2.2.6) leve a equação original

$$
\dot{x}=\varepsilon f^{1}(x, t)+\varepsilon^{2} f^{[2]}(x, t, \varepsilon)
$$

na equação promediada completa

$$
\dot{y}=\varepsilon \bar{f}^{1}(y)+\varepsilon^{2} f_{*}^{[2]}(y, t, \varepsilon),
$$

para alguma $f_{*}^{[2]}$, induzida pela mudança de coordenadas e periódica em $t$. Dessa forma, a equação promediada (ou equação promediada truncada)

$$
\dot{z}=\varepsilon \bar{f}^{1}(z)
$$

é obtida suprimindo o último termo de (2.2.8) e mudando o nome da variável de y para z. A nova variável $z$ não está relacionada a $x$ ou a $y$ por uma fórmula; em vez disso, $z$ é introduzida apenas para distinguir as soluções de (2.2.8) e (2.2.9).

A prova do Teorema 2.2.4 usando essas equações será dividida em lemas. O primeiro 
estabelece a validade das transformações próximas à identidade, o segundo, a existência de uma perturbação da identidade particular que precisaremos, e o terceiro estima o erro devido ao truncamento.

Lema 2.2.5. Consideremos (2.2.6) como uma aplicação

$$
y \mapsto U(y, t, \varepsilon)
$$

dependendo de $t$ e $\varepsilon$. Para qualquer aberto limitado e conexo $D \subset \mathbb{R}^{n}$, existe $\varepsilon_{0}$ tal que, para todo $t \in \mathbb{R}$ e para todo $\varepsilon$ satisfazendo $0 \leq \varepsilon \leq \varepsilon_{0}$, essa aplicação leva $D$ injetivamente em sua imagem $U(D, t, \varepsilon)$. A aplicação inversa tem a forma

$$
y=V(x, t, \varepsilon)=x+\varepsilon v^{[1]}(x, t, \varepsilon),
$$

e é suave em $(x, t, \varepsilon)$.

A seguinte prova usa o fato de que a aplicação $u^{[1]}$, presente na equação $(2.2 .6)$, é Lipschitz em $\bar{D}$ com alguma constante de Lipschitz $\lambda_{u^{[1]}}$. Alternativamente, o Lema 2.2.7 mostrará que se $f^{1}$ for Lipschitz com constante $\lambda_{f^{1}}$, então $u^{[1]}$ poderá ser tomada como sendo Lipschitz com constante $\lambda_{u^{[1]}}=2 \lambda_{f^{1}} T$.

Demonstração do Lema 2.2.5: Primeiramente, mostramos que $U$ é injetiva em $D$ para $\varepsilon$ suficientemente pequeno. Sejam $y_{1}, y_{2} \in D$ tais que

$$
U\left(y_{1}, t, \varepsilon\right)=U\left(y_{2}, t, \varepsilon\right)
$$

com $0 \leq \varepsilon \leq \frac{1}{\lambda_{u}[1]}$. Então, $y_{1}+\varepsilon u^{[1]}\left(y_{1}, t, \varepsilon\right)=y_{2}+\varepsilon u^{[1]}\left(y_{2}, t, \varepsilon\right)$

Assim,

$$
\begin{gathered}
y_{1}-y_{2}=-\varepsilon u^{[1]}\left(y_{1}, t, \varepsilon\right)+\varepsilon u^{[1]}\left(y_{2}, t, \varepsilon\right) \Rightarrow \\
\Rightarrow\left\|y_{1}-y_{2}\right\|=\varepsilon\left\|u^{[1]}\left(y_{1}, t, \varepsilon\right)-u^{[1]}\left(y_{2}, t, \varepsilon\right)\right\| \leq \varepsilon \lambda_{u^{[1]}}\left\|y_{1}-y_{2}\right\| .
\end{gathered}
$$

Se $\varepsilon \lambda_{u^{[1]}}<1$, acabamos de mostrar que, a menos que $\left\|y_{1}-y_{2}\right\|$ se anule, ele é menor do que si próprio. Portanto, $y_{1}=y_{2}$, e $U$ é injetiva para $0 \leq \varepsilon \leq \frac{1}{\lambda_{u^{[1]}}}$. Segue que $U$ aplica $D$ bijetivamente sobre $U(D, t, \varepsilon)$. Resta-nos verificar a suavidade e a forma da inversa. 
Como $\mathrm{D}_{y} U(y, t, 0)$ é a matriz identidade, o Teorema da Função Implícita implica que $x=U(y, t, \varepsilon)$ é localmente suavemente invertível na forma (2.2.10), para $\varepsilon$ suficientemente pequeno. Mais precisamente, para cada $y_{0} \in \mathbb{R}^{n}$ existe uma vizinhança na qual $U$ é invertível para $\varepsilon$ em um intervalo que depende de $y_{0}$. Como o fecho de $D$ é compacto, esse pode ser coberto por um número finito $k$ dessas vizinhanças, com raios $\varepsilon_{1}, \ldots, \varepsilon_{k} \mathrm{em}$ $\varepsilon$. Seja $\varepsilon_{0}=\min \left\{\frac{1}{\lambda_{u^{[1]}}}, \varepsilon_{1}, \ldots, \varepsilon_{k}\right\}$. Então, para $0 \leq \varepsilon \leq \varepsilon_{0}$, as inversas locais (que são suaves e têm a forma desejada) existem a devem coincidir com a inversa global.

Observação 2.2.6. Usamos a notação $\mathrm{D} f \cdot g$ para indicar a multiplicação de $g$ pela derivada de $f$ em relação à variável espacial.

Lema 2.2.7. Existe aplicação $U$ (não única) tal que a transformação (2.2.6) leva a equação (2.2.7) à equação (2.2.8). Em particular, $u^{[1]}$ pode ser tomada com constante de Lipschitz $2 \lambda_{f^{1}} T$ (onde $T$ é o período).

Demonstração: Se as equações $\dot{x}=\varepsilon f^{[1]}(x, t, \varepsilon)$ e $\dot{y}=\varepsilon g^{[1]}(y, t, \varepsilon)$ estiverem relacionadas pela mudança de coordenadas $x=U(y, t, \varepsilon)$, então pela Regra da Cadeia temos

$$
\dot{x}=\frac{\partial U}{\partial y} \cdot \frac{d y}{d t}+\frac{\partial U}{\partial t} \cdot \frac{d t}{d t} \Rightarrow \varepsilon f^{[1]}=\mathrm{D} U \cdot \varepsilon g^{[1]}+U_{t}
$$

ou mais rigorosamente

$$
f^{[1]}(U(y, t, \varepsilon), t, \varepsilon)=U_{t}(y, t, \varepsilon)+\mathrm{D} U(y, t, \varepsilon) \cdot g^{[1]}(y, t, \varepsilon) .
$$

Substituindo as formas de $U, f^{[1]}$ e $g^{[1]}$ dadas em (2.2.6), (2.2.7) e (2.2.8), temos:

$$
\begin{gathered}
\dot{x}=\dot{y}+\varepsilon \mathrm{D} u^{[1]}(y, t) \cdot \dot{y}+\varepsilon u_{t}^{[1]}(y, t) \Rightarrow \\
\Rightarrow \varepsilon f^{1}\left(y+\varepsilon u^{[1]}(y, t), t, \varepsilon\right)+\varepsilon^{2} f^{[2]}\left(y+\varepsilon u^{[1]}(y, t), t, \varepsilon\right)=\varepsilon \bar{f}^{1}(y)+\varepsilon^{2} f_{*}^{[2]}(y, t, \varepsilon)+ \\
+\varepsilon \mathrm{D} u^{[1]}(y, t) \cdot\left(\varepsilon \bar{f}^{1}(y)+\varepsilon^{2} f_{*}^{[2]}(y, t, \varepsilon)\right)+\varepsilon u_{t}^{[1]}(y, t),
\end{gathered}
$$

e extraindo o termo de maior ordem em $\varepsilon$ e fazendo $\varepsilon \rightarrow 0$, obtemos:

$$
u_{t}^{[1]}(y, t)=f^{1}(y, t)-\bar{f}^{1}(y) .
$$


A equação (2.2.11) é chamada de equação homológica do averaging.

Assumindo que a função $u^{[1]}$, como desejada, existe, a prova segue invertendo os passos. Consideremos (2.2.11) como uma equação a ser resolvida em $u^{[1]}$. Como o lado direito de (2.2.11) tem média zero, a função

$$
u^{[1]}(y, t)=\int_{0}^{t}\left[f^{1}(y, s)-\bar{f}^{1}(y)\right] d s+\kappa^{1}(y)
$$

será periódica em $t$ para qualquer escolha da função $\kappa^{1}$.

Agora, voltemos para o cálculo da regra da cadeia do início da demonstração, tomando $f^{[1]}$ como em $(2.2 .7), u^{[1]}$ como em $(2.2 .12)$, e considerando $g^{[1]}$ a ser determinada. Mostremos que $\dot{y}=\varepsilon g^{[1]}$ tem a forma $(2.2 .8)$ para alguma $f_{*}^{[2]}$.

De fato,

$$
\begin{gathered}
x=y+\varepsilon u^{[1]}(y, t) \Rightarrow \\
\Rightarrow x=y+\varepsilon\left[\int_{0}^{t}\left[f^{1}(y, s)-\bar{f}^{1}(y)\right] d s+\kappa^{1}(y)\right] \Rightarrow \\
\Rightarrow \dot{x}=\dot{y}+\varepsilon\left[f^{1}(y, t)-\bar{f}^{1}(y)\right]
\end{gathered}
$$

e, então,

$$
\begin{aligned}
& \varepsilon f^{1}\left(y+\varepsilon u^{[1]}(y, t), t\right)+\varepsilon^{2} f^{[2]}\left(y+\varepsilon u^{[1]}(y, t), t, \varepsilon\right)=\dot{y}+\varepsilon\left[f^{1}(y, t)-\bar{f}^{1}(y)\right] \Rightarrow \\
& \Rightarrow \dot{y}=\varepsilon \bar{f}^{1}(y)+\varepsilon\left[f^{1}\left(y+\varepsilon u^{[1]}(y, t), t\right)-f^{1}(y, t)\right]+\varepsilon^{2} f^{[2]}\left(y+\varepsilon u^{[1]}(y, t), t, \varepsilon\right) .
\end{aligned}
$$

Como $f^{1}(y, t)$ é a parte linear de $f^{1}\left(y+\varepsilon u^{[1]}(y, t), t\right)$ em relação a $\varepsilon$, então

$$
f^{1}\left(y+\varepsilon u^{[1]}(y, t), t\right)-f^{1}(y, t)=\mathcal{O}(\varepsilon) .
$$

Tomemos $f_{*}^{[2]}(y, t, \varepsilon)=\left[f^{1}\left(y+\varepsilon u^{[1]}(y, t), t\right)-f^{1}(y, t)\right]+\varepsilon f^{[2]}\left(y+\varepsilon u^{[1]}(y, t), t, \varepsilon\right)$.

Continuando a prova, vejamos agora que $\bar{f}^{1}$ tem a mesma constante de Lipschitz que 
$f^{1}$, a saber $\lambda_{f^{1}}$. Então, para $y_{1}, y_{2} \in D$,

$$
\begin{aligned}
\left\|\bar{f}^{1}\left(y_{1}\right)-\bar{f}^{1}\left(y_{2}\right)\right\| & =\left\|\frac{1}{T} \int_{0}^{T} f^{1}\left(y_{1}, s\right) d s-\frac{1}{T} \int_{0}^{T} f^{1}\left(y_{2}, s\right) d s\right\|= \\
& =\left\|\frac{1}{T} \int_{0}^{T}\left[f^{1}\left(y_{1}, s\right)-f^{1}\left(y_{2}, s\right)\right] d s\right\| \leq \\
& \leq \frac{1}{T} \int_{0}^{T}\left\|f^{1}\left(y_{1}, t\right)-f^{1}\left(y_{2}, t\right)\right\| d s \leq \\
& \leq \frac{1}{T} \int_{0}^{T} \lambda_{f^{1}}\left\|y_{1}-y_{2}\right\| d s= \\
& =\lambda_{f^{1}}\left\|y_{1}-y_{2}\right\| .
\end{aligned}
$$

Finalmente, verifiquemos que, se $\kappa^{1}(y)=0$, então $u^{[1]}$ terá constante de Lipschitz igual a $2 \lambda_{f^{1}} T$. De fato, como $u^{[1]}$ é periódica em $t$, para cada $t$ existe um $\tilde{t} \in[0, T]$ tal que

$$
u^{[1]}(y, t)=u^{[1]}(y, \tilde{t}) .
$$

Então,

$$
\begin{aligned}
\left\|u^{[1]}\left(y_{1}, t\right)-u^{[1]}\left(y_{2}, t\right)\right\| & =\left\|u^{[1]}\left(y_{1}, \tilde{t}\right)-u^{[1]}\left(y_{2}, \tilde{t}\right)\right\|= \\
& =\left\|\int_{0}^{\tilde{t}}\left[f^{1}\left(y_{1}, s\right)-\bar{f}^{1}\left(y_{1}\right)\right] d s-\int_{0}^{\tilde{t}}\left[f^{1}\left(y_{2}, s\right)-\bar{f}^{1}\left(y_{2}\right)\right] d s\right\|= \\
& =\left\|\int_{0}^{\tilde{t}}\left[f^{1}\left(y_{1}, s\right)-f^{1}\left(y_{2}, s\right)-\bar{f}^{1}\left(y_{1}\right)+\bar{f}^{1}\left(y_{2}\right)\right] d s\right\| \leq \\
& \leq \int_{0}^{\tilde{t}}\left[\left\|f^{1}\left(y_{1}, s\right)-f^{1}\left(y_{2}, s\right)\right\|+\left\|\bar{f}^{1}\left(y_{1}\right)-\bar{f}^{1}\left(y_{2}\right)\right\|\right] d s \leq \\
& \leq \int_{0}^{\tilde{t}}\left[\lambda_{f^{1}}\left\|y_{1}-y_{2}\right\|+\lambda_{\bar{f}^{1}}\left\|y_{1}-y_{2}\right\|\right] d s= \\
& =\left.2 \lambda_{f^{1}}\left\|y_{1}-y_{2}\right\| s\right|_{0} ^{\tilde{t}}=2 \lambda_{f^{1}}\left\|y_{1}-y_{2}\right\| \tilde{t} \leq 2 \lambda_{f^{1}}\left\|y_{1}-y_{2}\right\| T,
\end{aligned}
$$

o que prova o lema.

O modo mais simples de resolver a ambiguidade de (2.2.12) é escolher $\kappa^{1}(y)=0$. Fazendo esta escolha, teremos $U(y, 0, \varepsilon)=y$, de modo que as condições iniciais (em $t=0)$ não precisam ser transformadas quando se muda da coordenada $x$ para $y$. Além 
disso,

$$
U(y, m T, \varepsilon)=y
$$

em cada tempo estroboscópico $m T(m \in \mathbb{Z})$. Por essa razão, escolhendo $\kappa^{1}(y)=0$ temos o conhecido averaging estroboscópico.

Observação 2.2.8. A palavra "estroboscópio" é de origem grega, onde stróbos significa girar e skopéin, observar, e refere-se a um instrumento utilizado para estudar os corpos em movimento. Murdock [40] afirma que o termo "tempo estroboscópico" refere-se ao fato de que, se uma solução $\varphi(\alpha, t)$ de um campo vetorial, considerada como um ponto movendo-se no espaço $n$-dimensional, fosse iluminada pela luz de um estroboscópio nos tempos $0, T, 2 T, \ldots$, obteríamos a sequência de pontos $\alpha, \varphi(\alpha, T), \varphi(\alpha, 2 T), \ldots$ Esta sequência de pontos pode ser obtida como as iteradas da função $\Phi$ que aplica pontos em pontos, chamada de aplicação período. A saber, se $\Phi(\alpha)=\varphi(\alpha, t)$, então a sequência citada seria $\alpha, \Phi(\alpha), \Phi^{2}(\alpha), \ldots$

Agora, introduzimos as seguintes soluções específicas:

1. $x(t, \varepsilon)$ denota a solução de (2.2.7) com condição inicial $x(0, \varepsilon)=a$.

2. $y(t, \varepsilon)$ denota a solução de (2.2.8) com condição inicial

$$
y(0, \varepsilon)=V(a, 0, \varepsilon)=a+\varepsilon v^{[1]}(a, 0, \varepsilon)=a+\varepsilon b(\varepsilon) .
$$

Se o averaging estroboscópico for utilizado, (2.2.13) reduz-se a $y(0, \varepsilon)=a$. Notemos que

$$
x(t, \varepsilon)=U(y(t, \varepsilon), t, \varepsilon) .
$$

3. $z(t, \varepsilon)$ denota a solução de (2.2.9) com condição inicial $z(0, \varepsilon)=a$. Observemos o truncamento duplo envolvido aqui: tanto a equação diferencial quanto a condição inicial para $y$ (no caso não estroboscópico) foram truncadas para obter a equação diferencial e a condição inicial para $z$. Esta solução $z(t, \varepsilon)$ é tradicionalmente chamada de primeira aproximação para $x(t, \varepsilon)$. 
4. $U(z(t, \varepsilon), t, \varepsilon)$ é geralmente chamada de primeira aproximação melhorada para $x(t, \varepsilon)$. É natural considerar $z(t, \varepsilon)$ como uma aproximação para $y(t, \varepsilon)$, e em vista de (2.2.14) parece natural utilizar $U(z(t, \varepsilon), t, \varepsilon)$ como uma aproximação para $x(t, \varepsilon)$. Mas, $z(t, \varepsilon)$ já é uma aproximação de $\mathcal{O}(\varepsilon)$ para $x(t, \varepsilon)$ para tempo de $\mathcal{O}\left(\frac{1}{\varepsilon}\right)$. Aplicando $U$ temos uma mudança de $\mathcal{O}(\varepsilon)$ e, assim, a ordem da aproximação não é melhorada.

Os seguintes resultados estimam certas diferenças entre essas soluções.

Lema 2.2.9. As soluções $y(t, \varepsilon)$ e $z(t, \varepsilon)$ definidas acima satisfazem

$$
\|y(t, \varepsilon)-z(t, \varepsilon)\|=\mathcal{O}(\varepsilon)
$$

para tempo de $\mathcal{O}\left(\frac{1}{\varepsilon}\right)$.

\section{Demonstração: Temos}

$$
y(t, \varepsilon)=a+\varepsilon b(\varepsilon)+\int_{0}^{t}\left[\varepsilon \bar{f}^{1}(y(s, \varepsilon))+\varepsilon^{2} f_{*}^{[2]}(y(s, \varepsilon), s, \varepsilon)\right] d s
$$

$\mathrm{e}$

$$
z(t, \varepsilon)=a+\int_{0}^{t} \varepsilon \bar{f}^{1}(z(s, \varepsilon)) d s
$$

Fazendo $E(t, \varepsilon)=y(t, \varepsilon)-z(t, \varepsilon)$, segue que

$$
\begin{aligned}
\|E(t, \varepsilon)\| & =\left\|\varepsilon b(\varepsilon)+\int_{0}^{t} \varepsilon \bar{f}^{1}(y(s, \varepsilon)) d s+\int_{0}^{t} \varepsilon^{2} f_{*}^{[2]}(y(s, \varepsilon), s, \varepsilon) d s-\int_{0}^{t} \varepsilon \bar{f}^{1}(z(s, \varepsilon)) d s\right\| \leq \\
& \leq \varepsilon\|b(\varepsilon)\|+\varepsilon\left\|\int_{0}^{t}\left[\bar{f}^{1}(y(s, \varepsilon))-\bar{f}^{1}(z(s, \varepsilon))\right] d s\right\|+\varepsilon^{2}\left\|\int_{0}^{t} f_{*}^{[2]}(y(s, \varepsilon), s, \varepsilon) d s\right\| \leq \\
& \leq \varepsilon\|b(\varepsilon)\|+\varepsilon \lambda_{\bar{f}^{1}} \int_{0}^{t}\|E(s, \varepsilon)\| d s+\varepsilon^{2} M t
\end{aligned}
$$

onde $M$ é um limitante superior para $f_{*}^{[2]}$ em $\bar{D}$.

Aplicando o Lema de Gronwall Específico (Lema 2.2.3), obtemos

$$
\|E(t, \varepsilon)\| \leq \frac{\varepsilon^{2} M}{\varepsilon \lambda_{\bar{f}^{1}}+\varepsilon\|b(\varepsilon)\|} e^{\varepsilon \lambda_{\bar{f}^{1}} t}-\frac{\varepsilon^{2} M}{\varepsilon \lambda_{\bar{f}^{1}}}=\varepsilon\left(\frac{\varepsilon M}{\lambda_{\bar{f}^{1}}+\|b(\varepsilon)\|} e^{\varepsilon \lambda_{\bar{f}^{1}} t}-\frac{\varepsilon M}{\lambda_{\bar{f}^{1}}}\right) .
$$


Como o tempo é de $\mathcal{O}\left(\frac{1}{\varepsilon}\right)$, existe $L$ tal que $\varepsilon t \leq L$. Então,

$$
\|E(t, \varepsilon)\| \leq \varepsilon\left(\frac{\varepsilon M}{\lambda_{\bar{f}^{1}}+\|b(\varepsilon)\|} e^{\lambda_{\bar{f}^{1}} L}-\frac{\varepsilon M}{\lambda_{\bar{f}^{1}}}\right)
$$

o que prova o lema.

Teorema 2.2.10. As soluções $x(t, \varepsilon)$ e $z(t, \varepsilon)$ definidas acima satisfazem a estimativa

$$
\|x(t, \varepsilon)-z(t, \varepsilon)\|=\mathcal{O}(\varepsilon)
$$

para tempo de $\mathcal{O}\left(\frac{1}{\varepsilon}\right)$.

Demonstração: Pela desigualdade triangular,

$$
\|x(t, \varepsilon)-z(t, \varepsilon)\| \leq\|x(t, \varepsilon)-y(t, \varepsilon)\|+\|y(t, \varepsilon)-z(t, \varepsilon)\| .
$$

O primeiro termo é de $\mathcal{O}(\varepsilon)$ para todo tempo por (2.2.14) e (2.2.6), e o segundo termo é de $\mathcal{O}(\varepsilon)$ para tempo de $\mathcal{O}\left(\frac{1}{\varepsilon}\right)$ pelo Lema 2.2.9.

O Teorema 2.2.10 refere-se ao teorema do averaging de primeira ordem analítico e foi demonstrado seguindo as linhas de Bogoliubov. Definimos uma transformação de coordenadas que estivesse próxima à identidade e utilizamo-la para converter a equação original na equação promediada. Dessa forma, obtivemos uma solução (da equação promediada) que estivesse tão próxima quanto se quisesse da solução da equação original, para um intervalo de tempo longo, limitado e dependente de um parâmetro suficientemente pequeno.

A Figura 2.1 ilustra o comportamento das soluções do sistema original e do sistema promediado.

Uma importante variação do teorema básico do averaging lida com a uniformidade da estimativa com relação às condições iniciais: se $a$ variar em $D$, poderemos usar o mesmo c e o mesmo $L$ ? Uma resposta é dada pelo Teorema 2.2.12. Utilizamos a notação de [40]. A expressão $x(a, t, \varepsilon)$ denotará a solução $x(t, \varepsilon)$ de (2.2.1) com condição inicial $x(0)=a$.

No entanto, antes de enunciarmos e demonstrarmos o Teorema 2.2.12, enunciaremos o seguinte lema cuja demonstração encontra-se em [40], p. 151. 


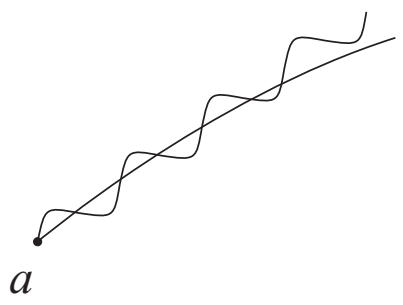

Figura 2.1: Ilustração esquemática das soluções do sistema original e do sistema promediado. A solução promediada de primeira ordem move-se lentamente ao longo de um arco suave, enquanto que a solução original sofre pequenas oscilações e afasta-se gradualmente.

Lema 2.2.11. Seja $r(t)$ uma função vetorial diferenciável de uma variável real. Então, $\|r(t)\|$ é diferenciável em cada ponto onde $r(t) \neq 0$, e diferenciável pela direita em todo ponto. Além disso, vale que

$$
\frac{d}{d t}\|r(t)\| \leq\left\|\frac{d}{d t} r(t)\right\|
$$

com a derivada da direita sendo usada quando necessária.

Teorema 2.2.12. Seja $K$ um subconjunto compacto de $\mathbb{R}^{n}$ e seja $D$ um subconjunto aberto, compacto e conexo contendo $K$ em seu interior. Seja $\varepsilon_{1}$ tal que a perturbação da identidade (2.2.6) seja válida (invertível) para $y \in D$ e $0 \leq \varepsilon \leq \varepsilon_{1}$. Seja $L>0$ arbitrário, e para cada $a \in K$, seja $L_{a}$ o maior número real que seja menor ou igual a $L$ tal que $w(a, \tau)(=z(a, t, \varepsilon))$ pertença $a K$ para $0 \leq \tau \leq L_{a}$. Então, existem c e $\varepsilon_{0}$ tais que

$$
\|x(a, t, \varepsilon)-z(a, t, \varepsilon)\|<c \varepsilon
$$

para $0 \leq t \leq \frac{L_{a}}{\varepsilon}$ e $0 \leq \varepsilon \leq \varepsilon_{0}$.

Demonstração: Como $D$ é compacto, todas as funções são suaves e todas as funções de $t$ que estão no lado direito de (2.2.6)-(2.2.9) são periódicas, segue que todas essas funções são limitadas e têm constantes de Lipschitz em D. Essas constantes serão introduzidas livremente quando forem necessárias nos seguintes argumentos.

Primeiramente, consideremos a solução $w(a, \tau)=z(a, t, \varepsilon)$ do sistema promediado para qualquer $a \in K$ fixo. Ou essa solução permanece em $K$ para todo tempo $\tau$, ou deixa $K$ em algum tempo $\tau=L_{a}^{1}$.

No primeiro caso, seja $L_{a}=L$ (onde $L$ é como na hipótese do teorema), e no último 
caso, seja $L_{a}$ o mínimo entre $L$ e $L_{a}^{1}$. Então, para $0 \leq \tau \leq L_{a}, w(a, \tau)$ pertence a $K$ e, assim, para $0 \leq t \leq \frac{L_{a}}{\varepsilon}, z(a, t, \varepsilon)$ pertence a $K$. Para entender a construção desse intervalo de tempo, notemos que, se $a$ estiver próximo à fronteira de $K$, então $L_{a}$ provavelmente será inferior a $L$ porque a solução do sistema promediado pode sair de $K$ antes que $\tau$ atinja $L$, enquanto que, se $a$ estiver "distante" da fronteira de $K$, então $L_{a}$ será provavelmente $L$, já que esse limite absoluto no tempo $\tau$ possivelmente será alcançado antes que $w$ atinja a fronteira. Se todas as soluções do sistema promediado passarem por $K$ no tempo $\tau$ menor do que $L$, então todos os valores de $L_{a}$ serão menores do que $L$. Se, por outro lado, o campo vetorial lento apontar para dentro de $K$ em todos os pontos da fronteira, então todas as soluções da equação lenta permanecerão em $K$ para todo tempo. Nesse caso, $L_{a}$ será igual a $L$ para todo $a$. Mesmo nesse caso não é (em geral) possível estender a estimativa do erro (2.2.16) para todo tempo, e embora $L$ seja arbitrário, é necessário ter um tal limite para obter constantes $c$ e $\varepsilon_{0}$ adequadas.

O próximo passo na demonstração é estimar a diferença entre $y$ e $z$, soluções de (2.2.8) e (2.2.9), respectivamente. Sejam $\lambda_{\bar{f}^{1}}$ a constante de Lipschitz de $\bar{f}^{1}$ em $D$ e $M$ um limitante superior para $\left\|f_{*}^{[2]}(y, t, \varepsilon)\right\|$, para $y \in D, 0 \leq t \leq T$ e $0 \leq \varepsilon \leq \varepsilon_{1}$. Então, por (2.2.8), (2.2.9), pela desigualdade triangular e pelo Lema 2.2.11, temos:

$$
\begin{aligned}
\frac{d}{d t}\|y(a, t, \varepsilon)-z(a, t, \varepsilon)\| & \leq\|\dot{y}(a, t, \varepsilon)-\dot{z}(a, t, \varepsilon)\|= \\
& =\left\|\varepsilon \bar{f}^{1}(y)+\varepsilon^{2} f_{*}^{[2]}(y, t, \varepsilon)-\varepsilon \bar{f}^{1}(z)\right\| \leq \\
& \leq \varepsilon\left\|\bar{f}^{1}(y)-\bar{f}^{1}(z)\right\|+\varepsilon^{2}\left\|f_{*}^{[2]}(y, t, \varepsilon)\right\| \leq \\
& \leq \varepsilon \lambda_{\bar{f}^{1}}\|y(a, t, \varepsilon)-z(a, t, \varepsilon)\|+\varepsilon^{2} M,
\end{aligned}
$$

o que implica que

$$
\|y(a, t, \varepsilon)-z(a, t, \varepsilon)\| \leq \varepsilon \lambda_{\bar{f}^{1}} \int_{0}^{t}\|y(a, s, \varepsilon)-z(a, s, \varepsilon)\| d s+\varepsilon^{2} M t .
$$

Pelo Lema de Gronwall Específico (Lema 2.2.3),

$$
\|y(a, t, \varepsilon)-z(a, t, \varepsilon)\| \leq \varepsilon \frac{M}{\lambda_{\bar{f}^{1}}}\left(e^{\varepsilon \lambda_{\bar{f}^{1}} t}-1\right),
$$


enquanto $y$ e $z$ permanecerem em $D$. Já que $z$ permanece em $K$ (e, consequentemente, em $D)$ para $0 \leq t \leq \frac{L_{a}}{\varepsilon}$, e uma vez que o lado direito de (2.2.17) é crescente em $t$, segue que

$$
\|y(a, t, \varepsilon)-z(a, t, \varepsilon)\| \leq \varepsilon \frac{M}{\lambda_{\bar{f}^{1}}}\left(e^{\lambda_{\bar{f}^{1}} L_{a}}-1\right) \leq \varepsilon \frac{M}{\lambda_{\bar{f}^{1}}}\left(e^{\lambda_{\bar{f}^{1}} L}-1\right)=c_{1} \varepsilon
$$

enquanto $y$ permanecer em $D$ e $0 \leq t \leq \frac{L_{a}}{\varepsilon}$, $\operatorname{com} c_{1}=\frac{M}{\lambda_{\bar{f}^{1}}}\left(e^{\lambda_{\bar{f}^{1}} L}-1\right)$.

Agora, escolhamos $\varepsilon_{0}$ de forma que $0 \leq \varepsilon_{0} \leq \varepsilon_{1}$ e $\varepsilon_{0} \leq \frac{d}{c_{1}}$, onde $d$ é a menor distância entre as fronteiras de $K$ e $D$. Para $\varepsilon$ no intervalo $0 \leq \varepsilon \leq \varepsilon_{0}$, consideremos as soluções para $y$ e $z$ começando em $a \in K$. A estimativa (2.2.18) vale de $t=0$ até $t=\frac{L_{a}}{\varepsilon}$ ou até $y$ deixar $D$, o qual ocorrer primeiro. Mas, desde que $z$ pertença a $K$ até $t=\frac{L_{a}}{\varepsilon}$ e desde que $c_{1} \varepsilon<d$, a estimativa (2.2.18) impede que $y$ se afaste suficientemente de $z$ para deixar $D$ antes do tempo $\frac{L_{a}}{\varepsilon}$. Portanto, (2.2.18) é válida para $0 \leq t \leq \frac{L_{a}}{\varepsilon}$ e $0 \leq \varepsilon \leq \varepsilon_{0}$. Pela teoria geral de existência de soluções de equações diferenciais, a solução para $y$ somente pode deixar de existir após deixar o compacto $D$. Portanto, essa solução existe pelo menos até o tempo $\frac{L_{a}}{\varepsilon}$.

O último passo nesta prova é comparar $x \operatorname{com} y$. Mas, isso segue do fato dessas soluções estarem relacionadas pela transformação de mudança de coordenadas (2.2.6). Denotemos por $c_{2}$ o valor máximo de $\left\|u^{[1]}(y, t)\right\|$, para $y \in D$ e $0 \leq t \leq T$. Então, é claro que

$$
\|x(a, t, \varepsilon)-y(a, t, \varepsilon)\|=\left\|\varepsilon u^{[1]}(y, t)\right\| \leq c_{2} \varepsilon
$$

com $y \in D$ e $0 \leq \varepsilon \leq \varepsilon_{1}$. Contudo, já sabemos que $y$ pertence a $D$ quando $0 \leq t \leq \frac{L_{a}}{\varepsilon}$ e $0 \leq \varepsilon \leq \varepsilon_{0}$.

Fazendo $c=c_{1}+c_{2}$ e colocando (2.2.18) e (2.2.19) juntas, obtemos (2.2.16).

O Teorema 2.2.12 forneceu-nos um critério do tipo de uniformidade que a estimativa de erro possui com respeito à condição inicial $a$. Façamos, agora, uma nova demonstração do Teorema 2.2.10, mas visto como uma consequência do Teorema 2.2.12.

Outra demonstração para o Teorema 2.2.10: Lembremo-nos que, neste teorema, a condição inicial $a$ e o limitante superior do tempo $L$ são dados fixados. 
Sejam $K$ o conjunto dos pontos traçados por $z(a, t, \varepsilon)$, para $0 \leq t \leq \frac{L}{\varepsilon}$, e $D$ um conjunto compacto contendo $K$ em seu interior. Então, $L$ é precisamente $L_{a}$, como definido no Teorema 2.2.12, para o valor inicial $a$; isto é, $L$ é a quantidade de tempo $\tau=\varepsilon t$ para a qual a solução inicia-se em $a$ e permanece em $K$. Portanto, a equação (2.2.15) é apenas um caso especial de (2.2.16).

Guckenheimer e Holmes em [21] complementam o Teorema 2.2.10 com o seguinte resultado.

Teorema 2.2.13. [21] Se $p_{0}$ for um ponto hiperbólico fixado de (2.2.2), então existirá um $\varepsilon_{0}>0$ tal que, para todo $0 \leq \varepsilon \leq \varepsilon_{0}$, a equação (2.2.1) terá uma única órbita periódica hiperbólica $\gamma_{\varepsilon}(t)=p_{0}+\mathcal{O}(\varepsilon)$ de mesma estabilidade de $p_{0}\left(\gamma_{\varepsilon}\right.$ poderá ser uma órbita periódica trivial, $\left.\gamma_{\varepsilon}(t)=p_{0}\right)$.

Demonstração: A demonstração deste teorema pode ser encontrada em [21].

Exemplo 2.2.14. [21] Consideremos o sistema

$$
\dot{x}=\varepsilon f(x, t, \varepsilon)=\varepsilon x \operatorname{sen}^{2} t .
$$

Reescrevemos a equação (2.2.20) na forma $\dot{x}=\varepsilon\left(\frac{x}{2}-\frac{x}{2} \cos 2 t\right)$. Definindo $u^{[1]}(y, t)$ de forma que $\frac{\partial u^{[1]}}{\partial t}(y, t)=-\frac{y}{2} \cos 2 t$, isto é,

$$
u^{[1]}(y, t)=-\frac{y}{4} \operatorname{sen} 2 t
$$

a equação (2.2.6) fica definida como

$$
x=U(y, t, \varepsilon)=y-\frac{y}{4} \operatorname{sen} 2 t
$$

Logo, a equação promediada completa,

$$
\dot{y}=\varepsilon \bar{f}(y)+\varepsilon^{2}\left[\mathrm{D}_{y} f(y, t, 0) u^{[1]}(y, t, 0)-\mathrm{D}_{y} u^{[1]}(y, t, 0) \bar{f}(y)+\frac{\partial \tilde{f}}{\partial \varepsilon}(y, t, 0)\right]+\mathcal{O}\left(\varepsilon^{3}\right),
$$


tem a expressão dada por

$$
\dot{y}=\varepsilon \frac{y}{2}+\varepsilon^{2} \frac{y}{16} \operatorname{sen} 4 t+\mathcal{O}\left(\varepsilon^{3}\right) .
$$

Notemos que a equação promediada truncada autônoma é simplesmente

$$
\dot{z}=\varepsilon \frac{z}{2}
$$

Vemos que a solução exata de (2.2.20) com condição inicial $x(0)=a$ tem a expressão

$$
x(t)=a e^{\varepsilon\left(\frac{t}{2}-\frac{\operatorname{sen} 2 t}{4}\right)} .
$$

Comparando (2.2.24) com a solução da equação promediada truncada,

$$
z(t)=a e^{\varepsilon \frac{t}{2}}, z(0)=a,
$$

observamos que

$$
\begin{aligned}
\|x(t)-z(t)\| & =\left\|a e^{\varepsilon\left(\frac{t}{2}-\frac{\operatorname{sen} 2 t}{4}\right)}-a e^{\varepsilon \frac{t}{2}}\right\|=\left\|e^{\varepsilon \frac{t}{2}}\left[a e^{-\varepsilon \frac{\operatorname{sen} 2 t}{4}}-a\right]\right\|= \\
& =\left\|(1+\mathcal{O}(\varepsilon))\left(-\varepsilon \frac{1}{4} a \operatorname{sen} 2 t+\mathcal{O}\left(\varepsilon^{2}\right)\right)\right\|=\mathcal{O}(\varepsilon) .
\end{aligned}
$$

Pelo Teorema 2.2.13, a fonte hiperbólica $z=0$ de (2.2.23) corresponde à órbita periódica hiperbólica trivial $x \equiv 0$ de (2.2.20). A Figura 2.2 ilustra os retratos de fase das equações (2.2.20) e (2.2.23), enquanto que a Figura 2.3 exibe os gráficos das soluções (2.2.24) e $(2.2 .25)$.

\subsection{A forma padrão}

Vimos na Seção 2.2 que os resultados envolvendo o método do averaging de primeira ordem foram todos construídos e demonstrados para equações na forma padrão

$$
\dot{x}=\varepsilon f^{1}(x, t)+\cdots+\varepsilon^{k} f^{k}(x, t)+\varepsilon^{k+1} f^{[k+1]}(x, t, \varepsilon),
$$




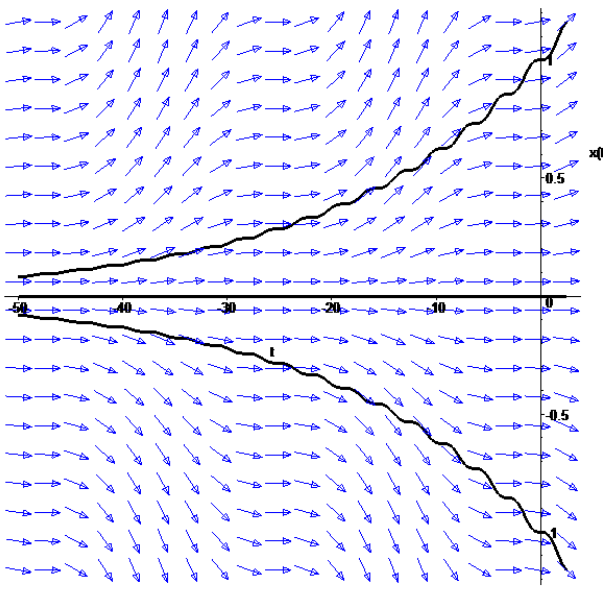

(a)

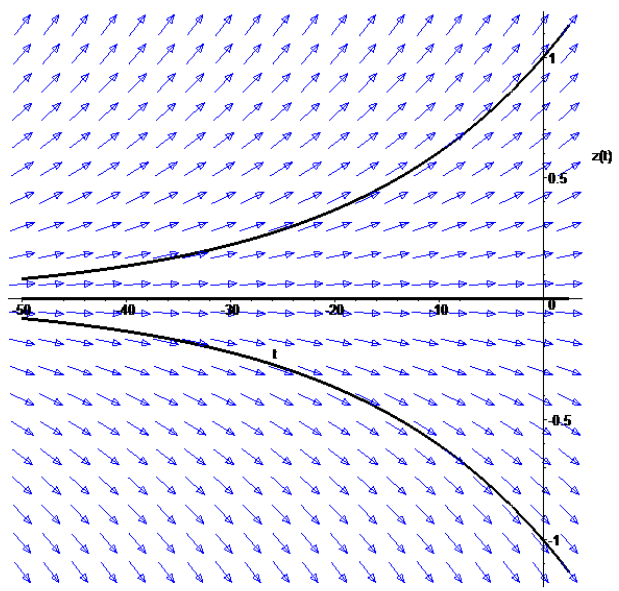

(b)

Figura 2.2: (a) Retrato de fase da equação (2.2.20) com condição inicial $x(0)=1 \mathrm{e}$ $\varepsilon=0,1$. (b) Retrato de fase da equação (2.2.23) com condição inicial $z(0)=1$ e $\varepsilon=0,1$.

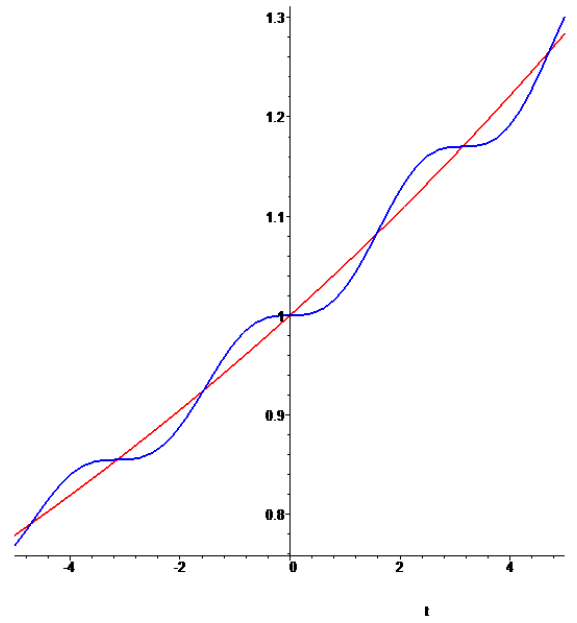

Figura 2.3: O traço em azul indica a solução (2.2.24) e o traço em vermelho, a solução (2.2.25). Observemos que a solução $x(t)$ oscila à medida que $z(t)$ movimenta-se lentamente.

onde $x \in \mathbb{R}^{n}, t \in \mathbb{R}$ e $\varepsilon>0$ é um parâmetro pequeno.

Nesta seção, estabelecemos a necessidade e importância da equação estar na forma padrão para estudá-la através do método do averaging.

É muito comum depararmo-nos com equações que não estejam em tal forma. O objetivo desta seção é abordar algumas técnicas utilizadas para escrever uma equação diferencial na forma padrão, salvo algumas particularidades. Tais métodos estão descritos em [41].

Com a finalidade de estudarmos tais métodos, alguns exemplos são abordados. No 
entanto, vale ressaltar que nesta seção não estamos focados a exemplos de equações que tenham singularidades somente do tipo centro, pois, a princípio, estamos preocupados em estudar as transformações que colocarão as equações na forma padrão.

\subsubsection{A reformulação na forma padrão}

Consideremos um problema de perturbação da forma

$$
\dot{x}=f^{0}(x, t)+\varepsilon f^{[1]}(x, t, \varepsilon), x\left(t_{0}\right)=a,
$$

e o problema não perturbado

$$
\dot{z}=f^{0}(z, t), z\left(t_{0}\right)=a \text {. }
$$

Assumamos que (2.3.3) possa ser resolvido explicitamente. Como a solução dependerá do valor inicial $a$, denotemos por $z(\xi, t)$ a solução de (2.3.3) com condição inicial $\xi$, isto é,

$$
z=z(\xi, t), z\left(\xi, t_{0}\right)=\xi, \xi \in \mathbb{R}^{n} .
$$

Agora, consideremos a equação acima como uma transformação (método da variação dos parâmetros ou variação das constantes):

$$
x=z(\xi, t) .
$$

Usando (2.3.2) e (2.3.3) e derivando a equação diferencial com relação a $\xi$, teremos

$$
\frac{\partial}{\partial t} z(\xi, t)+\mathrm{D}_{\xi} z(\xi, t) \frac{d \xi}{d t}=f^{0}(z(\xi, t), t)+\varepsilon f^{[1]}(z(\xi, t), t, \varepsilon)
$$

Como $z$ satisfaz a equação não perturbada, os primeiros termos de ambos os lados da igualdade cancelam-se. Assumindo que $\mathrm{D}_{\xi} z(\xi, t)$ é uma matriz não singular, temos

$$
\dot{\xi}=\varepsilon\left(\mathrm{D}_{\xi} z(\xi, t)\right)^{-1} \cdot f^{[1]}(z(\xi, t), t, \varepsilon) .
$$


A equação (2.3.5) é chamada de problema de perturbação na forma padrão. Notemos que esse sistema está escrito na forma de (2.3.1).

Apesar de ser chamada "forma padrão", a equação obtida não é simples e de fácil estudo e nem sempre nos leva a uma equação cuja solução seja uma boa aproximação para o sistema desejado. Os próximos exemplos visam explicar tal comentário.

Exemplo 2.3.1. Consideremos a equação do pêndulo perturbado

$$
\ddot{\phi}+\operatorname{sen} \phi=\varepsilon g(\phi, t, \varepsilon) \text {. }
$$

Nesse caso, a equação (2.3.5) necessariamente envolverá funções elípticas, pois as soluções da equação não perturbada $\ddot{\phi}+\operatorname{sen} \phi=0$ é composta por funções elípticas, o que dificulta os cálculos.

Exemplo 2.3.2. Consideremos duas espécies vivendo em uma região com uma oferta restrita de alimentos e uma pequena interação entre elas afetando sua densidade populacional $x_{1}$ e $x_{2}$. Descrevemos o crescimento populacional pelo modelo

$$
\left\{\begin{array}{l}
\dot{x}_{1}=\beta_{1} x_{1}-x_{1}^{2}+\varepsilon f_{1}\left(x_{1}, x_{2}\right), x_{1}(0)=a_{1}, \\
\dot{x}_{2}=\beta_{2} x_{2}-x_{2}^{2}+\varepsilon f_{2}\left(x_{1}, x_{2}\right), x_{2}(0)=a_{2},
\end{array}\right.
$$

onde as constantes $\beta_{i}, a_{i}>0$ e $x_{i}(t) \geq 0$ para $i=1,2$. Consideremos o sistema não perturbado na variável $z=\left(z_{1}, z_{2}\right)$. Sua solução é dada por

$$
z_{i}(t)=\frac{\beta_{i} a_{i} e^{\beta_{i} t}}{\beta_{i}+a_{i}\left(e^{\beta_{i} t}-1\right)}, i=1,2
$$

Queremos calcular a inversa da matriz

$$
\mathrm{D}_{\xi} z(\xi, t)=\left(\begin{array}{cc}
\frac{\partial z_{1}(t)}{\partial \xi_{1}} & \frac{\partial z_{1}(t)}{\partial \xi_{2}} \\
\frac{\partial z_{2}(t)}{\partial \xi_{1}} & \frac{\partial z_{2}(t)}{\partial \xi_{2}}
\end{array}\right), \xi_{i}(0)=a_{i}, i=1,2
$$


Notemos que $\frac{\partial z_{1}(t)}{\partial \xi_{2}}=\frac{\partial z_{2}(t)}{\partial \xi_{1}}=0$ e que

$$
\frac{\partial z_{i}(t)}{\partial \xi_{i}}=\frac{\left(\beta_{i} e^{\beta_{i} t}\right)\left(\beta_{i}+\xi_{i}\left(e^{\beta_{i} t}-1\right)\right)-\beta_{i} \xi_{i} e^{\beta_{i} t}\left(e^{\beta_{i} t}-1\right)}{\left(\beta_{i}+\xi_{i}\left(e^{\beta_{i} t}-1\right)\right)^{2}}=\frac{e^{\beta_{i} t}}{\left(1+\frac{\xi_{i}}{\beta_{i}}\left(e^{\beta_{i} t}-1\right)\right)^{2}}, i=1,2
$$

Assim, aplicando (2.3.5), obtemos

$$
\dot{\xi}_{i}=\varepsilon e^{-\beta_{i} t}\left(1+\frac{\xi_{i}}{\beta_{i}}\left(e^{\beta_{i} t}-1\right)\right)^{2} f_{i}(\cdot, \cdot), i=1,2
$$

na qual abreviamos a expressão para $f_{i}$.

Essa transformação não teve um efeito prático neste exemplo, pois o lado direito da expressão cresce exponencialmente, mesmo se tomarmos $f_{i}$ constante. O crescimento exponencial implica que quanto maior for o tempo, mais rápido a solução crescerá, e isso poderá implicar no afastamento entre as soluções do sistema não perturbado e do perturbado, o que não é a finalidade do método do averaging.

Existe uma classe de problemas na qual essa técnica funciona adequadamente e tratamos dela na Seção 2.3.2.

\subsubsection{A forma padrão no caso quasilinear}

O problema de perturbação (2.3.2) será chamado quasilinear se a equação puder ser escrita como

$$
\dot{x}=A(t) x+\varepsilon f^{[1]}(x, t, \varepsilon),
$$

na qual $A(t)$ é uma matriz $n \times n$ contínua. Nesse caso, se o problema não perturbado

$$
\dot{y}=A(t) y
$$

possuir $n$ soluções linearmente independentes, construiremos a matriz fundamental $\Phi(t)$, de forma que $\Phi\left(t_{0}\right)=I$ (matriz identidade de ordem $n$ ) e aplicaremos o processo de variação das constantes, chamando

$$
x=\Phi(t) z
$$


Assim obtemos, a partir de (2.3.5),

$$
\dot{z}=\varepsilon \Phi^{-1}(t) f^{[1]}(\Phi(t) z, t, \varepsilon) .
$$

Se $A$ for uma matriz constante para todo $t$, a matriz fundamental terá a forma

$$
\Phi(t)=e^{A\left(t-t_{0}\right)}
$$

A forma padrão neste caso fica

$$
\dot{z}=\varepsilon e^{-A\left(t-t_{0}\right)} f^{[1]}\left(e^{A\left(t-t_{0}\right)} z, t, \varepsilon\right) .
$$

Observação 2.3.3. Vale observar que, se os autovalores de $A$ não forem todos imaginários puros, podemos não conseguir uma limitação para $e^{-A\left(t-t_{0}\right)}$ e, mesmo que $f^{[1]}$ seja limitada, a equação perturbada (2.3.8) poderá apresentar soluções que não sejam limitadas.

Na teoria de osciladores não lineares forçados o problema de perturbação pode ser da forma

$$
\dot{x}=f^{0}(x, t)+\varepsilon f^{[1]}(x, t, \varepsilon),
$$

onde $f^{0}(x, t)=A x+h(t)$, com $A$ uma matriz constante. A transformação da variação das constantes torna-se

$$
x=e^{A\left(t-t_{0}\right)} z+e^{A\left(t-t_{0}\right)} \int_{t_{0}}^{t} e^{-A\left(s-t_{0}\right)} h(s) d s .
$$

E, então, o problema de perturbação na forma padrão é escrito como

$$
\dot{z}=\varepsilon e^{-A\left(t-t_{0}\right)} f^{[1]}(x, t, \varepsilon),
$$

na qual $x$ ainda precisa ser substituído pela equação (2.3.10).

Vejamos um exemplo no qual utilizamos a transformação da variação das constantes tal qual a descrevemos nesta seção.

Exemplo 2.3.4. Ao estudar osciladores não lineares, frequentemente consideramos o 
problema de valor inicial perturbado

$$
\ddot{x}+\omega^{2} x=\varepsilon g(x, \dot{x}, t, \varepsilon), x\left(t_{0}\right)=a_{1}, \dot{x}\left(t_{0}\right)=a_{2} .
$$

Duas soluções independentes do problema não perturbado, $\ddot{y}+\omega^{2} y=0$, são $\cos \left(\omega\left(t-t_{0}\right)\right)$ e $\operatorname{sen}\left(\omega\left(t-t_{0}\right)\right)$. Dessa forma, a matriz fundamental é dada por

$$
\Phi(t)=\left(\begin{array}{cc}
\cos \left(\omega\left(t-t_{0}\right)\right) & \frac{1}{\omega} \operatorname{sen}\left(\omega\left(t-t_{0}\right)\right) \\
-\omega \operatorname{sen}\left(\omega\left(t-t_{0}\right)\right) & \cos \left(\omega\left(t-t_{0}\right)\right)
\end{array}\right) .
$$

Observemos que esta matriz é tal que $\Phi\left(t_{0}\right)=I$. Então, a transformação da variação das constantes torna-se

$$
\left\{\begin{array}{l}
x=z_{1} \cos \left(\omega\left(t-t_{0}\right)\right)+\frac{z_{2}}{\omega} \operatorname{sen}\left(\omega\left(t-t_{0}\right)\right), \\
\dot{x}=-z_{1} \omega \operatorname{sen}\left(\omega\left(t-t_{0}\right)\right)+z_{2} \cos \left(\omega\left(t-t_{0}\right)\right) .
\end{array}\right.
$$

A equação na forma padrão (2.3.8) neste caso é

$$
\left\{\begin{array}{l}
\dot{z}_{1}=-\frac{\varepsilon}{\omega} \operatorname{sen}\left(\omega\left(t-t_{0}\right)\right) g(\cdot, \cdot, t, \varepsilon), z_{1}\left(t_{0}\right)=a_{1} \\
\dot{z}_{2}=\varepsilon \cos \left(\omega\left(t-t_{0}\right)\right) g(\cdot, \cdot, t, \varepsilon), \quad z_{2}\left(t_{0}\right)=a_{2}
\end{array}\right.
$$

onde as expressões para $x$ e $\dot{x}$ de (2.3.12) devem ser substituídas em $g$, nos pontos.

Vejamos outra maneira de utilizar soluções independentes do sistema e o processo da variação das constantes utilizando equações que permitam a variação da amplitude $r$ e da fase $\phi$ da solução de um sistema linear. Definindo $x=r \operatorname{sen}(\omega t-\phi)$, então $\dot{x}=y=r \omega \cos (\omega t-\phi)$ e obtemos a chamada transformação amplitude-fase. Dessa forma as equações perturbadas serão

$$
\left(\begin{array}{c}
\dot{r} \\
\dot{\phi}
\end{array}\right)=\varepsilon\left(\begin{array}{c}
\frac{1}{\omega} \cos (\omega t-\phi) g(\cdot, \cdot, t, \varepsilon) \\
\frac{1}{r \omega} \operatorname{sen}(\omega t-\phi) g(\cdot, \cdot, t, \varepsilon)
\end{array}\right) \text {. }
$$

Os valores iniciais para $r$ e $\phi$ podem ser calculados pela substituição anterior.

Nos Exemplos 2.3.5 e 2.3.7 utilizaremos as transformações acima descritas. 
Exemplo 2.3.5. Consideremos a equação diferencial de segunda ordem

$$
\ddot{x}+x=\varepsilon g(x, \dot{x}),
$$

com valores iniciais $x_{0}$ e $\dot{x}_{0}$ dados, e $g$ uma função suficientemente suave em $D \subset \mathbb{R}^{2}$. Esse é um sistema quasilinear e usaremos a transformação amplitude-fase para colocar (2.3.15) na forma padrão. Sejam

$$
\left\{\begin{array}{l}
x=r \operatorname{sen}(t-\phi) \\
\dot{x}=r \cos (t-\phi)
\end{array}\right.
$$

Dessa forma, as equações perturbadas (2.3.14) ficam

$$
\left(\begin{array}{c}
\dot{r} \\
\dot{\phi}
\end{array}\right)=\varepsilon\left(\begin{array}{c}
\cos (t-\phi) g(r \operatorname{sen}(t-\phi), r \cos (t-\phi)) \\
\frac{1}{r} \operatorname{sen}(t-\phi) g(r \operatorname{sen}(t-\phi), r \cos (t-\phi))
\end{array}\right) \text {, }
$$

e notemos que ela está na forma

$$
\dot{x}=\varepsilon f^{1}(x, t),
$$

$\operatorname{com} x=(r, \phi)$.

Além disso, observemos que o campo vetorial é $2 \pi$-periódico em $t$ e, como visto no Teorema 2.2.4, se $g \in \mathcal{C}^{1}(D)$, poderemos calcular a média de $f^{1}$, exceto para uma vizinhança da origem (onde as coordenadas polares falham). Como a equação original é autônoma, a equação promediada dependerá somente de $r$, e as duas componentes do campo vetorial promediado são

$$
\begin{aligned}
\bar{f}_{1}^{1}(r) & =\frac{1}{2 \pi} \int_{0}^{2 \pi} \cos (s-\phi) g(r \operatorname{sen}(s-\phi), r \cos (s-\phi)) d s= \\
& =\frac{1}{2 \pi} \int_{0}^{2 \pi} \cos (s) g(r \operatorname{sen}(s), r \cos (s)) d s
\end{aligned}
$$

e

$$
\bar{f}_{2}^{1}(r)=\frac{1}{r} \frac{1}{2 \pi} \int_{0}^{2 \pi} \operatorname{sen}(s) g(r \operatorname{sen}(s), r \cos (s)) d s .
$$


Uma aproximação assintótica pode ser obtida resolvendo o sistema

$$
\left\{\begin{aligned}
\dot{\bar{r}} & =\varepsilon \bar{f}_{1}^{1}(\bar{r}) \\
\dot{\bar{\phi}} & =\varepsilon \bar{f}_{2}^{1}(\bar{r})
\end{aligned}\right.
$$

com valores iniciais apropriados.

Se $g(x, \dot{x})=\left(1-x^{2}\right) \dot{x}$, temos a conhecida equação de Van der Pol

$$
\ddot{x}+x=\varepsilon\left(1-x^{2}\right) \dot{x},
$$

sendo neste caso

$$
\left\{\begin{aligned}
\dot{\bar{r}} & =\frac{1}{2} \varepsilon \bar{r}\left(1-\frac{1}{4} \bar{r}^{2}\right) \\
\dot{\bar{\phi}} & =0
\end{aligned}\right.
$$

Se o valor inicial da amplitude $r_{0}$ for 0 ou 2, a amplitude $\bar{r}$ será constante para todo o tempo. O valor $r_{0}=0$ corresponde ao ponto crítico instável na origem, enquanto que $r_{0}=2$ fornece uma solução periódica dada pela expressão

$$
\bar{r}(t)=2 \operatorname{sen}\left(t-\phi_{0}\right)+\mathcal{O}(\varepsilon)
$$

no tempo escala $\frac{1}{\varepsilon}$. Portanto, obtemos a solução geral

$$
\bar{r}(t)=\frac{r_{0} e^{\frac{1}{2} \varepsilon t}}{\left(1+\frac{1}{4} r_{0}^{2}\left(e^{\varepsilon t}-1\right)\right)^{\frac{1}{2}}} \operatorname{sen}\left(t-\phi_{0}\right)+\mathcal{O}(\varepsilon)
$$

no tempo escala $\frac{1}{\varepsilon}$. Além disso, as soluções tendem para a solução periódica (2.3.17) que é um ciclo limite (estável). A Figura 2.4 traz seu retrato de fase.

Observação 2.3.6. Com o estudo apresentado acima, podemos fazer uma comparação dos resultados obtidos com a aplicação das duas técnicas estudadas nesta dissertação para a investigação dos ciclos limites que bifurcam de um centro. No Exemplo 1.4.10, aplicamos o método das integrais abelianas na equação de Van der Pol, obtendo a existência de um ciclo limite bifurcando-se da circunferência de raio $\sqrt{2}$. No Exemplo 2.3.5, aplicamos o método do averaging e constatamos a existência de um ciclo limite estável cuja equação 


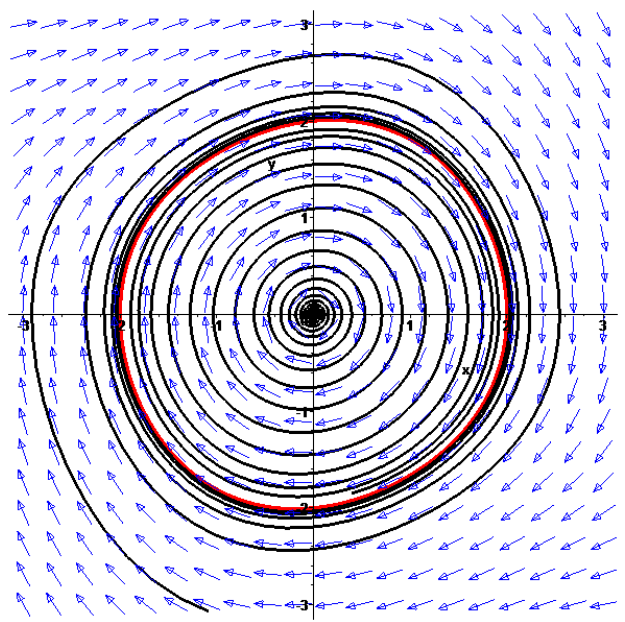

Figura 2.4: Retrato de fase da equação de Van der Pol, $\ddot{x}+x=\varepsilon\left(1-x^{2}\right) \dot{x}$, com $\varepsilon=0,1$. A origem é um ponto crítico instável do fluxo; o ciclo limite (curva fechada, em vermelho) corresponde à solução periódica estável.

aproximada é dada por

$$
\bar{r}(t)=2 \operatorname{sen}\left(t+\phi_{0}\right)+\mathcal{O}(\varepsilon)
$$

no tempo escala $\frac{1}{\varepsilon}$.

Dessa forma, aplicando ambos os métodos, obtemos o mesmo número de ciclos limites que bifurcam da equação $\ddot{x}+x=0$ após uma perturbação do tipo $g(x, \dot{x})=\left(1-x^{2}\right) \dot{x}$, com a vantagem de que no método do averaging obtemos a expressão analítica de uma aproximação da solução periódica.

Passemos, agora, para o último exemplo desta seção.

Exemplo 2.3.7. Consideremos a equação de um oscilador linear com modulação de frequência, também conhecida como equação de Mathieu, dada por

$$
\ddot{x}+(1+2 \varepsilon \cos (2 t)) x=0
$$

com valores iniciais $x(0)=x_{0}$ e $\dot{x}(0)=\dot{x}_{0}$. Podemos proceder como no Exemplo 2.3.5; no entanto, a equação (2.3.15) agora depende explicitamente do tempo $t$. Nessa última 
equação, tomando $g(x, \dot{x})=2 \cos (2 t) x$, a transformação amplitude-fase fornece-nos

$$
\left\{\begin{array}{l}
\dot{r}=-2 \varepsilon r \operatorname{sen}(t-\phi) \cos (t-\phi) \cos (2 t), \\
\dot{\phi}=-2 \varepsilon \operatorname{sen}^{2}(t-\phi) \cos (2 t) .
\end{array}\right.
$$

O lado direito da equação acima é $2 \pi$-periódica em $t$. Assim, calculando a média dessas funções periódicas com respeito a $t$, obtemos o sistema

$$
\left\{\begin{aligned}
\dot{\bar{r}} & =\frac{1}{2} \varepsilon \bar{r} \operatorname{sen}(2 \bar{\phi}) \\
\dot{\bar{\phi}} & =\frac{1}{2} \varepsilon \cos (2 \bar{\phi})
\end{aligned}\right.
$$

Com o intuito de aproximar as soluções de um sistema linear que seja dependente do tempo (equação (2.3.19)), teremos de resolver um sistema autônomo não linear (equação (2.3.20)). Nesse caso a integração pode ser realizada, mas é mais prático escolhermos uma transformação diferente para obter a forma padrão, permanecendo ainda na categoria de sistemas lineares com transformações lineares. Utilizemos, então, a transformação (2.3.12) $\operatorname{com} \omega=1$ e $t_{0}=0$ :

$$
\left\{\begin{array}{l}
x=z_{1} \cos t+z_{2} \operatorname{sen} t \\
\dot{x}=-z_{1} \operatorname{sen} t+z_{2} \cos t
\end{array}\right.
$$

Logo, a equação (2.3.13) fica na forma

$$
\left\{\begin{array}{l}
\dot{z}_{1}=2 \varepsilon \operatorname{sen} t \cos (2 t)\left(z_{1} \cos t+z_{2} \operatorname{sen} t\right) \\
\dot{z}_{2}=-2 \varepsilon \cos t \cos (2 t)\left(z_{1} \cos t+z_{2} \operatorname{sen} t\right)
\end{array}\right.
$$

As equações do lado direito são $2 \pi$-periódicas, e o averaging fornece-nos

$$
\left\{\begin{array}{lll}
\dot{\bar{z}}_{1} & =-\frac{1}{2} \varepsilon \bar{z}_{2}, & \bar{z}_{1}(0)=x_{0} \\
\dot{\bar{z}}_{2}=-\frac{1}{2} \varepsilon \bar{z}_{1}, & \bar{z}_{2}(0)=\dot{x}_{0} .
\end{array}\right.
$$

Esse é um sistema linear cujas soluções são

$$
\left\{\begin{array}{l}
\bar{z}_{1}(t)=\frac{1}{2}\left(x_{0}+\dot{x}_{0}\right) e^{-\frac{1}{2} \varepsilon t}+\frac{1}{2}\left(x_{0}-\dot{x}_{0}\right) e^{\frac{1}{2} \varepsilon t} \\
\bar{z}_{2}(t)=\frac{1}{2}\left(x_{0}+\dot{x}_{0}\right) e^{-\frac{1}{2} \varepsilon t}-\frac{1}{2}\left(x_{0}-\dot{x}_{0}\right) e^{\frac{1}{2} \varepsilon t}
\end{array}\right.
$$


A aproximação assintótica para a solução $x(t)$ da equação de Mathieu é

$$
\bar{x}(t)=\frac{1}{2}\left(x_{0}+\dot{x}_{0}\right)(\cos t+\operatorname{sen} t)+\frac{1}{2}\left(x_{0}-\dot{x}_{0}\right)(\cos t-\operatorname{sen} t) .
$$

A Figura 2.5 mostra o gráfico de $\bar{x}(t)$ para valores inicias específicos.

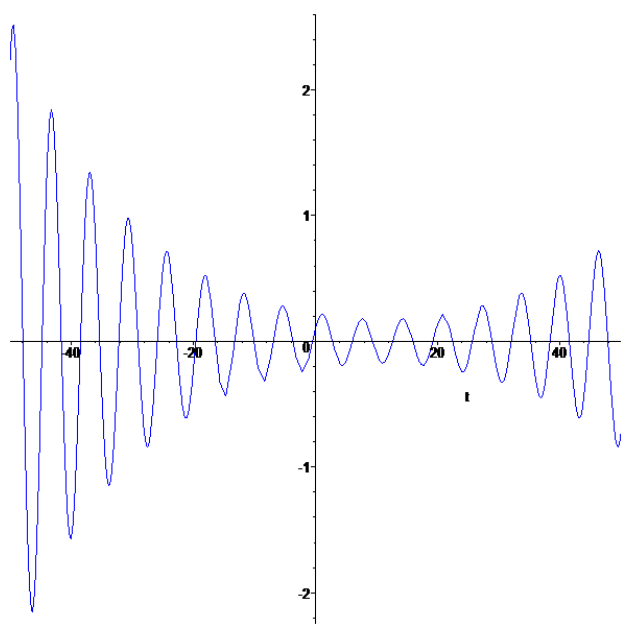

Figura 2.5: Gráfico da solução aproximada $\bar{x}(t)$ para $x_{0}=0,1 ; \dot{x}_{0}=0,2$ e $\varepsilon=0,1$.

Notemos que o equilíbrio $x=\dot{x}=0$ é instável.

\subsubsection{A importância da forma padrão}

Até o momento analisamos exemplos nos quais estivemos preocupados em transpô-los para a forma padrão. Segundo Murdock [40], é absolutamente essencial para a utilização do averaging que o sistema original esteja na forma padrão. Para que nos convençamos disto, consideremos o problema de valor inicial

$$
\begin{cases}\dot{x}_{1}=1, & x_{1}(0)=0, \\ \dot{x}_{2}=\varepsilon \cos \left(x_{1}-t\right), & x_{2}(0)=0\end{cases}
$$

que não está na forma padrão.

Este sistema tem solução exata $x_{1}(t)=t, x_{2}(t)=\varepsilon t$. Se calcularmos o sistema promediado, obteremos

$$
\left\{\begin{array}{l}
\dot{z}_{1}=1, \quad z_{1}(0)=0 \\
\dot{z}_{2}=0, \quad z_{2}(0)=0
\end{array}\right.
$$


A teoria do averaging

com solução exata $z_{1}(t)=t, z_{2}(t)=0$. O erro na segunda componente é $\varepsilon t$, que é igual a 1 quando $t=\frac{1}{\varepsilon}$; se o averaging fosse válido para este sistema (da forma que é válido para sistemas na forma padrão), o erro nesse tempo seria da ordem de $\varepsilon$ (isto é, $\mathcal{O}(\varepsilon)$ ).

O problema está na primeira equação de (2.3.21). Nela, $x_{1}$ varia com o tempo de tal forma que $\cos \left(x_{1}-t\right)$ permanece em seu valor máximo 1, que não é bem aproximado pelo seu valor médio 0. Isso cria uma diferença de $\varepsilon$ entre os lados direitos das segundas equações de (2.3.21) e (2.3.22). Embora essa discrepância seja pequena, ela gera um erro em $z_{2}$ que cresce para 1 no tempo $\frac{1}{\varepsilon}$.

Se $\dot{x}_{1}$ fosse $\varepsilon$ ao invés de $1,(2.3 .21)$ estaria na forma padrão. Nesse caso, $x_{1}$ variaria lentamente e, durante o período $0 \leq t \leq 2 \pi$, o termo $\cos \left(x_{1}-t\right)$ comportaria-se como se $x_{1}$ fosse constante: realizaria uma oscilação quase senoidal e seria melhor aproximada pelo seu valor médio 0 comparado ao caso atual (2.3.21).

Portanto, a importância da forma padrão periódica é que para $\varepsilon$ suficientemente pequeno, $x$ é quase constante, de modo que faz sentido calcular a média do lado direito da equação ao longo de um período em que $x$ mantém-se constante.

Observação 2.3.8. Nos Exemplos 2.3.4, 2.3.5 e 2.3.7, discutimos e analisamos alguns casos particulares da equação polinomial generalizada de Liénard, a saber

$$
\ddot{x}+f(x) \dot{x}+g(x)=0,
$$

onde $f$ e $g$ são polinômios na variável $x$ de graus $n$ e $m$, respectivamente, nos quais queríamos obter uma forma padrão da equação utilizando algumas transformações, com a finalidade de aplicarmos o método do averaging.

Há uma vasta quantidade de publicações envolvendo algum estudo sobre a equação de Liénard, e dentre elas existem os trabalhos envolvendo o estudo de suas soluções periódicas. Por exemplo, Gasull, Giacomini e Llibre [20] desenvolveram um novo critério para verificar a existência ou não de ciclos limites da equação de Liénard via o controle do sinal de uma função de uma variável.

Além disso, existem também trabalhos que trazem uma análise da quantidade de ciclos limites da equação de Liénard utilizando o método do averaging, como Mereu [39] e 
Llibre, Mereu e Teixeira [34], que aplicaram o método do averaging de primeira, segunda e terceira ordens à equação polinomial generalizada de Liénard. Na Seção 2.5 apresentamos alguns exemplos estudados por Mereu em [39] através do método do averaging.

Após o estudo do método do averaging de primeira ordem e dessa breve discussão sobre a forma padrão e sua importância, voltemos nossa atenção ao método do averaging de alta ordem. Na Seção 2.4 adaptamos os resultados vistos na Seção 2.2 para equações diferenciais que possuem ordem mais elevada na variável $\varepsilon$ e investigamos quão próximas estão as soluções dos sistema original e as soluções do sistema promediado para intervalos de tempo mais longos.

\subsection{Averaging periódico de alta ordem analítico}

O averaging de ordem $k$ descrito nesta seção pode ser utilizado para obter estimativas de erro de $\mathcal{O}\left(\varepsilon^{k}\right)$ válidas para tempo de $\mathcal{O}\left(\frac{1}{\varepsilon}\right)$, com $k>1$, e (sob suposições mais restritas) obter estimativas de erro de $\mathcal{O}\left(\varepsilon^{k-j}\right)$ (mais fraco) para tempo de $\mathcal{O}\left(\frac{1}{\varepsilon^{j+1}}\right)$ (mais longo). No último caso, diremos que trocamos $j$ ordens de exatidão por um maior tempo de validade.

Nesta dissertação tratamos do primeiro caso e enunciamos o Teorema 2.4.3 que trata do segundo caso. Os resultados desta seção podem ser encontrados em [41].

Consideremos todas as funções suaves e definidas em $\mathbb{R}^{n}$. Como no averaging de primeira ordem, existem duas maneiras de demonstrar o teorema principal. A primeira demonstração é devida a Ellison, Sáenz e Dumas [17] e utiliza a desigualdade de Besjes (Lema 2.2.1), e a última é mais tradicional e segue as linhas de Bogoliubov. No entanto, desta vez, ambas as provas utilizam transformações próximas à identidade.

O próximo lema generaliza o Lema 2.2.7 e é formulado para incluir o que for necessário para ambas as provas.

Lema 2.4.1. Dado o sistema

$$
\dot{x}=\varepsilon f^{1}(x, t)+\cdots+\varepsilon^{k} f^{k}(x, t)+\varepsilon^{k+1} f^{[k+1]}(x, t, \varepsilon),
$$


com período $T$ em $t$, existe uma transformação

$$
x=U(y, t, \varepsilon)=y+\varepsilon u^{1}(y, t)+\varepsilon^{k} u^{k}(y, t),
$$

também periódica, tal que

$$
\dot{y}=\varepsilon g^{1}(y)+\cdots+\varepsilon^{k} g^{k}(y)+\varepsilon^{k+1} g^{[k+1]}(y, t, \varepsilon) .
$$

Aqui, $g^{1}$ é igual à média $\bar{f}^{1}$ de $f^{1}$, e $g^{2}, \ldots, g^{k}$ são independentes de $t$, mas não são únicas (já que elas dependem de escolhas feitas para obter os $u^{i}$ ). Existe um algoritmo para calcular essas funções na seguinte ordem: $g^{1}, u^{1}, g^{2}, u^{2}, \ldots, g^{k}, u^{k}$. Em particular, é possível calcular a equação promediada truncada (autônoma)

$$
\dot{z}=\varepsilon g^{1}(z)+\cdots+\varepsilon^{k} g^{k}(z)
$$

sem calcular o último termo de (2.4.2). Se a transformação "menor"

$$
\xi=\hat{U}(z, t, \varepsilon)=z+\varepsilon u^{1}(z, t)+\cdots+\varepsilon^{k-1} u^{k-1}(z, t)
$$

for aplicada a (2.4.4), o resultado será a seguinte modificação da equação original, na qual $h^{k}(\cdot, t)$ tem média zero:

$$
\dot{\xi}=\varepsilon f^{1}(\xi, t)+\cdots+\varepsilon^{k}\left[f^{k}(\xi, t)+h^{k}(\xi, t)\right]+\varepsilon^{k+1} \tilde{f}^{[k+1]}(\xi, t, \varepsilon) .
$$

Demonstração: Indicaremos os passos da demonstração. Como no Lema 2.2.5, a transformação (2.4.2) é invertível e define uma mudança de coordenadas. Quando essa mudança de coordenadas for aplicada a (2.4.1), o resultado terá a forma (2.4.3), exceto que, em geral, $g^{j}$ dependerá de $t$. Os cálculos são complicados e estão melhores descritos na Seção 3.2 de [41]; mas para cada $j$, o resultado tem a forma $g^{j}=K^{j}+\frac{\partial u^{j}}{\partial t}$, onde $K^{j}$ é uma função construída a partir de $f^{1}, \ldots, f^{j}$, de $u^{1}, \ldots, u^{j-1}$ previamente calculadas, e de 
suas derivadas. As duas primeiras funções $K^{j}$ são dadas por

$$
\left(\begin{array}{c}
K^{1}(y, t) \\
K^{2}(y, t)
\end{array}\right)=\left(\begin{array}{c}
f^{1}(y, t) \\
f^{2}(y, t)+\mathrm{D}_{y} f^{1}(y, t) \cdot u^{1}(y, t)-\mathrm{D}_{y} u^{1}(y, t) \cdot g^{1}(y)
\end{array}\right) .
$$

(Essa expressão recursiva assume que $g^{1}$ tenha sido calculada antes que $K^{2}$ seja obtida. Isto é, $g^{1}$ pode ser substituída por $f^{1}-u_{t}^{1}$ ). Assim, se $g^{j}$ for independente de $t$, teremos

$$
\frac{\partial u^{j}}{\partial t}(y, t)=K^{j}(y, t)-g^{j}(y)
$$

Essa equação homológica tem a mesma forma que (2.2.11) e pode ser resolvida da mesma maneira: tomemos $g^{j}=\bar{K}^{j}$ de forma que o lado direito de (2.4.8) tenha média zero e integremos com respeito a $t$. Isso determina $u^{j}$, a menos de uma "constante" $\kappa^{2}(y)$; após o primeiro caso $j=1$ (onde $K^{1}=f^{1}$ e a equação homológica é a mesma do averaging de primeira ordem), as constantes previamente escolhidas compõem $K^{j}$, fazendo com que $g^{j}$ não seja única. O restante da demonstração consiste em inverter os passos e checar que, com $g^{j}$ e $u^{j}$ construídas dessa maneira, a transformação (2.4.2) realmente leva (2.4.1) em (2.4.3), para alguma $g^{[k+1]}$. No caso de (2.4.5) e (2.4.6), a última equação homológica é substituída por

$$
0=K^{k}+h^{k}-g^{k}
$$

(Isso depende da estrutura interna de $K^{k}$. Na verdade, $K^{k}$ é igual a $f^{k}$ mais termos independentes de $f^{k}$, de modo que, quando $h^{k}$ for adicionada a $f^{k}$, também será adicionada a $K^{k}$ ). Tomando $g^{k}=\bar{K}^{k}$, segue que $h^{k}=K^{k}-\bar{K}^{k}$ tem média zero.

Escolhendo $\kappa^{j}(y)=0$, para todos os $j$, mais uma vez temos o averaging estroboscópico, no qual ambas as transformações $U$ e $\hat{U}$ reduzem-se à identidade nos tempos estroboscópicos. Se o averaging estroboscópico for utilizado, a maneira mais natural de construir uma aproximação para a solução $x(a, t, \varepsilon)$ de (2.4.1) com valor inicial $x(a, 0, \varepsilon)=a$ será resolver a equação promediada truncada (2.4.4), com $z(a, 0, \varepsilon)=a$, e aplicar essa solução $z(a, t, \varepsilon)$ na transformação $\hat{U}$ para obter

$$
\xi(a, t, \varepsilon)=\hat{U}(z(a, t, \varepsilon), t, \varepsilon) .
$$


Esta é uma importante diferença entre o averaging de primeira ordem e o de ordem maior: não é possível utilizar $z(a, t, \varepsilon)$ diretamente como uma aproximação para $x(a, t, \varepsilon)$. (No caso de primeira ordem, não precisamos definir $\hat{U}$ porque ela reduz-se à identidade). Se o averaging estroboscópico não for utilizado, a equação em $z$ deverá ser resolvida não com condição inicial $a$, mas com uma condição inicial que seja dependente de $\varepsilon$, $V(a, 0, \varepsilon)$, onde $y=V(x, t, \varepsilon)$ é a aplicação inversa da mudança de coordenadas $U$. (Não é necessário calcular a expressão exata de $V$, mas somente sua série de potências em $\varepsilon$ de ordem suficiente, que pode ser feito recursivamente).

Nas demonstrações seguintes assumimos o averaging estroboscópico por conveniência, mas os teoremas continuam válidos no caso geral.

Teorema 2.4.2. A solução exata $x(a, t, \varepsilon)$ e sua aproximação $\xi(a, t, \varepsilon)$, definida acima, estão relacionadas por

$$
\|x(a, t, \varepsilon)-\xi(a, t, \varepsilon)\|=\mathcal{O}\left(\varepsilon^{k}\right)
$$

para tempo de $\mathcal{O}\left(\frac{1}{\varepsilon}\right)$ e $\varepsilon$ pequeno.

Demonstração: Para a demonstração utilizando a desigualdade de Besjes, escrevemos

$$
\mathrm{J}_{\varepsilon}^{k} f^{[1]}=\varepsilon f^{1}(x, t)+\cdots+\varepsilon^{k} f^{k}(x, t)
$$

de forma que (2.4.6) fica reescrita como

$$
\dot{\xi}=\mathrm{J}_{\varepsilon}^{k} f^{[1]}(\xi, t, \varepsilon)+\varepsilon^{k} h^{k}(\xi, t)+\varepsilon^{k+1} \tilde{f}^{[k+1]}(\xi, t, \varepsilon) .
$$

Seja $E(t, \varepsilon)=x(a, t, \varepsilon)-\xi(a, t, \varepsilon)$. Então,

$$
\begin{aligned}
\|E(t, \varepsilon)\|= & \|x(a, t, \varepsilon)-\xi(a, t, \varepsilon)\|= \\
= & \| \int_{0}^{t}\left[\varepsilon f^{1}(x, s)+\cdots+\varepsilon^{k} f^{k}(x, s)+\varepsilon^{k+1} f^{[k+1]}(x, s, \varepsilon)\right] d s- \\
& -\int_{0}^{t}\left[\mathrm{~J}_{\varepsilon}^{k} f^{[1]}(\xi, s, \varepsilon)+\varepsilon^{k} h^{k}(\xi, s)+\varepsilon^{k+1} \tilde{f}^{[k+1]}(\xi, s, \varepsilon)\right] d s \|=
\end{aligned}
$$




$$
\begin{aligned}
= & \| \int_{0}^{t}\left[\mathrm{~J}_{\varepsilon}^{k} f^{[1]}(x, s)-\mathrm{J}_{\varepsilon}^{k} f^{[1]}(\xi, s, \varepsilon)\right] d s-\int_{0}^{t} \varepsilon^{k} h^{k}(\xi, s) d s+ \\
& +\int_{0}^{t} \varepsilon^{k+1}\left[f^{[k+1]}(x, s, \varepsilon)-\tilde{f}^{[k+1]}(\xi, s, \varepsilon)\right] d s \| \leq \\
\leq & \varepsilon \lambda_{\mathrm{J}_{\varepsilon}^{k}} f^{[1]} \int_{0}^{t}\|E(s, \varepsilon)\| d s+\varepsilon^{k}\left\|\int_{0}^{t} h^{k}(\xi, s) d s\right\|+ \\
& +\varepsilon^{k+1} \int_{0}^{t}\left\|f^{[k+1]}(x, s, \varepsilon)-\tilde{f}^{[k+1]}(\xi, s, \varepsilon)\right\| d s .
\end{aligned}
$$

Pelos mesmos argumentos utilizados na demonstração do Teorema 2.2.4, existem constantes $c_{0}$ e $c_{1}$ tais que

$$
\|E(t, \varepsilon)\| \leq \varepsilon \lambda_{J_{\varepsilon}^{k} f[1]} \int_{0}^{t}\|E(s, \varepsilon)\| d s+c_{0} \varepsilon^{k} t+c_{1} \varepsilon^{k+1}
$$

Pelo Lema 2.2.3,

$$
\begin{aligned}
\|E(t, \varepsilon)\| & \leq \frac{c_{0} \varepsilon^{k}}{\varepsilon \lambda_{J_{\varepsilon}^{k} f^{[1]}}+c_{1} \varepsilon^{k+1}} e^{\varepsilon \lambda_{J_{\varepsilon}^{k} f} f^{[1]} t}-\frac{c_{0} \varepsilon^{k}}{\varepsilon \lambda_{J_{\varepsilon}^{k} f^{[1]}}}= \\
& =\varepsilon^{k-1}\left(\frac{c_{0}}{\lambda_{J_{\varepsilon}^{k} f^{[1]}}+c_{1} \varepsilon^{k}} e^{\varepsilon \lambda_{J_{\varepsilon}^{k} f^{[1]}} t}-\frac{c_{0}}{\lambda_{J_{\varepsilon}^{k} f^{[1]}}}\right) .
\end{aligned}
$$

Para a conclusão da prova faz-se necessário aplicar os resultados de [17]. Este trabalho tem por objetivo demonstrar que a estimativa de ordem $k$, enunciada neste teorema, pode ser alcançada a partir da estimativa de ordem $k-1$ obtida em (2.4.10).

Façamos, agora, a demonstração tradicional ao longo das ideias do Lema 2.2 .9 e Teorema 2.2.10. Segue do Lema de Gronwall Específico que

$$
\|y(a, t, \varepsilon)-z(a, t, \varepsilon)\|=\mathcal{O}\left(\varepsilon^{k}\right)
$$

para tempo de $\mathcal{O}\left(\frac{1}{\varepsilon}\right)$.

Agora, escrevendo $U(y)$ para $U(y(a, t, \varepsilon), t, \varepsilon)$ e similarmente para outras expressões, temos $x=U(y), \xi=\hat{U}(z)$ e, portanto,

$$
\|x-\xi\|=\|U(y)-\hat{U}(z)\| \leq\|U(y)-\hat{U}(y)\|+\|\hat{U}(y)-\hat{U}(z)\|
$$


Notemos que

$$
\|U(y)-\hat{U}(y)\|=\left\|\varepsilon^{k} u^{k}(y, t)\right\|=\varepsilon^{k}\left\|u^{k}(y, t)\right\|=\mathcal{O}\left(\varepsilon^{k}\right),
$$

já que $u^{k}(y, t)$ é limitada em $\bar{D}$ para tempo de $\mathcal{O}\left(\frac{1}{\varepsilon}\right)$, e que

$$
\|\hat{U}(y)-\hat{U}(z)\| \leq\|y-z\|=\mathcal{O}\left(\varepsilon^{k}\right)
$$

pela afirmação anterior.

O resultado segue.

Por conveniência, enunciamos novamente esse teorema no caso de segunda ordem com fórmulas completas. Começando com o sistema

$$
\dot{x}=\varepsilon f^{1}(x, t)+\varepsilon^{2} f^{2}(x, t)+\varepsilon^{3} f^{[3]}(x, t, \varepsilon),
$$

coloquemos $g^{1}(y)=\bar{f}^{1}(y)$. Então, seja

$$
u^{1}(y, t)=\int_{0}^{t}\left[f^{1}(y, s)-g^{1}(y)\right] d s,
$$

definamos $K^{2}$ como em (2.4.7) e seja $g^{2}(y)=\bar{K}^{2}(y)$. Seja $z(t, \varepsilon)$ a solução de

$$
\dot{z}=\varepsilon g^{1}(z)+\varepsilon^{2} g^{2}(z), \quad z(0)=a .
$$

Então, a solução do problema original é

$$
x(t, \varepsilon)=z(t, \varepsilon)+\varepsilon u^{1}(z(t, \varepsilon), t)+\mathcal{O}\left(\varepsilon^{2}\right)
$$

para tempo de $\mathcal{O}\left(\frac{1}{\varepsilon}\right)$.

Para obter estimativas para intervalos de tempo mais longos, é necessário assumir que parte das equações promediadas anulam-se.

Teorema 2.4.3. Com a notação do Lema 2.4.1, suponhamos que $g^{1}=g^{2}=\cdots=g^{\ell-1}=$ 
$=0$, onde $\ell \leq k$. Então, as soluções de (2.4.1), (2.4.3) e (2.4.4) existem para tempo de $\mathcal{O}\left(\frac{1}{\varepsilon^{\ell}}\right)$ e, para cada inteiro $j=0,1, \ldots, \ell-1$, a solução exata $x(a, t, \varepsilon)$ e a solução aproximada $\xi(a, t, \varepsilon)$ definida por (2.4.9) satisfazem a estimativa

$$
\|x(a, t, \varepsilon)-\xi(a, t, \varepsilon)\|=\mathcal{O}\left(\varepsilon^{k-j}\right)
$$

para tempo de $\mathcal{O}\left(\frac{1}{\varepsilon^{j+1}}\right)$.

Demonstração: Para uma demonstração deste resultado veja [41].

O caso mais importante do Teorema 2.4.3 é quando $\ell=k$ e $j=\ell-1$. Nesse caso, a equação (2.4.4) reduz-se a $\dot{z}=\varepsilon^{\ell} g^{\ell}(z)$ e estamos trocando o mais de exatidão possível pelo aumento do tempo de validade, de forma que o erro seja de $\mathcal{O}(\varepsilon)$ para tempo de $\mathcal{O}\left(\frac{1}{\varepsilon^{\ell}}\right)$.

Exemplo 2.4.4. [41] Consideremos a equação de Van der Pol modificada definida por

$$
\ddot{x}+x-\varepsilon x^{2}=\varepsilon^{2}\left(1-x^{2}\right) \dot{x},
$$

a qual é equivalente ao sistema

$$
\left\{\begin{array}{l}
\dot{x}=y \\
\dot{y}=-x+\varepsilon x^{2}+\varepsilon^{2}\left(1-x^{2}\right) y
\end{array}\right.
$$

Escolhemos a transformação amplitude-fase com $\omega=1$, isto é,

$$
(x, y) \mapsto(r, \phi), \operatorname{com} x=r \operatorname{sen}(t-\phi) \text { e } y=r \cos (t-\phi),
$$

para obter a equação perturbada na forma padrão:

$$
\left\{\begin{array}{l}
\dot{r}=\varepsilon r^{2} \cos (t-\phi) \operatorname{sen}^{2}(t-\phi)+\varepsilon^{2} r \cos ^{2}(t-\phi)\left[1-r^{2} \operatorname{sen}^{2}(t-\phi)\right] \\
\dot{\phi}=\varepsilon r \operatorname{sen}^{3}(t-\phi)+\varepsilon^{2} \operatorname{sen}(t-\phi) \cos (t-\phi)\left[1-r^{2} \operatorname{sen}^{2}(t-\phi)\right]
\end{array}\right.
$$


Se definirmos

$$
f^{1}(r, \phi)=\left(\begin{array}{c}
f_{1}^{1}(r, \phi) \\
f_{2}^{1}(r, \phi)
\end{array}\right)=\left(\begin{array}{c}
r^{2} \cos (t-\phi) \operatorname{sen}^{2}(t-\phi) \\
r \operatorname{sen}^{3}(t-\phi)
\end{array}\right)
$$

$\mathrm{e}$

$$
f^{2}(r, \phi)=\left(\begin{array}{c}
r \cos ^{2}(t-\phi)\left[1-r^{2} \operatorname{sen}^{2}(t-\phi)\right] \\
\operatorname{sen}(t-\phi) \cos (t-\phi)\left[1-r^{2} \operatorname{sen}^{2}(t-\phi)\right]
\end{array}\right)
$$

vemos que a hipótese do Teorema 2.4.3 está satisfeita para $\ell=k=2$ e $j=1$, pois $g^{1}(r, \phi, t)=\bar{f}^{1}(r, \phi)=0$. Dessa forma, a equação promediada descreve um fluxo no tempo escala $\frac{1}{\varepsilon^{2}}$ com um erro de $\mathcal{O}(\varepsilon)$. Pela equação (2.4.11), obtemos

$$
u^{1}(r, \phi, t)=\left(\begin{array}{c}
\frac{1}{3} r^{2} \operatorname{sen}^{3}(t-\phi) \\
-\frac{1}{3} r \cos (t-\phi)\left(2+\operatorname{sen}^{2}(t-\phi)\right)
\end{array}\right)
$$

Um cálculo simples nos fornece

$$
\mathrm{D} f^{1}(r, \phi, t)=\left(\begin{array}{cc}
2 r \cos (t-\phi) \operatorname{sen}^{2}(t-\phi) & r^{2} \operatorname{sen}(t-\phi)\left[\operatorname{sen}^{2}(t-\phi)-2 \cos ^{2}(t-\phi)\right] \\
\operatorname{sen}^{3}(t-\phi) & -3 r \cos (t-\phi) \operatorname{sen}^{2}(t-\phi)
\end{array}\right)
$$

Após o cálculo de $\mathrm{D} f^{1} \cdot u^{1}, K^{2}$ pela expressão (2.4.7) e calcular a média, obtemos

$$
\left\{\begin{aligned}
\dot{\bar{r}} & =\frac{1}{2} \varepsilon^{2} \bar{r}\left(1-\frac{1}{4} \bar{r}^{2}\right) \\
\dot{\bar{\phi}} & =\frac{5}{12} \varepsilon^{2} \bar{r}^{2}
\end{aligned}\right.
$$

Então, concluímos que, como na equação de Van der Pol, temos uma solução periódica estável com amplitude $r=2+\mathcal{O}(\varepsilon)$. A função do termo de $\mathcal{O}(\varepsilon)$ na equação original é somente induzir um deslocamento do ângulo de fase $\phi$. Para uma aproximação da solução periódica temos a expressão

$$
\bar{r}(t)=2 \cos \left(t-\frac{5}{3} \varepsilon^{2} t\right)+\mathcal{O}(\varepsilon)
$$

no tempo escala $\frac{1}{\varepsilon^{2}}$. A Figura 2.6 ilustra o retrato de fase de (2.4.12). 


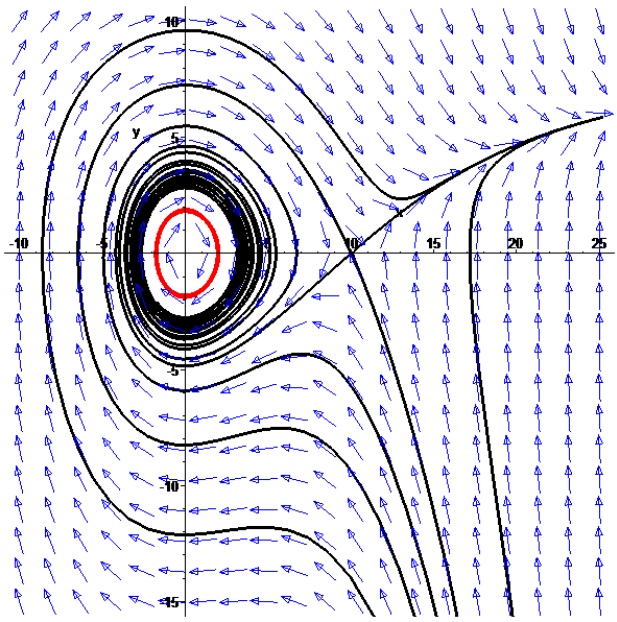

Figura 2.6: Retrato de fase da equação (2.4.12) para $\varepsilon=0,1$.

O método do averaging discutido até esta seção foi de caráter analítico, no qual foram utilizados os conceitos de aproximação assintótica para realizar as estimativas de erro das soluções do sistema original com as soluções do sistema promediado. Entretanto, há outro tipo de abordagem para o método do averaging: a topológica. Nessa versão do averaging precisamos do conceito do grau de Brouwer.

\subsection{Averaging periódico via grau de Brouwer}

Do estudo realizado até esta seção, podemos dizer que o método do averaging fornece uma relação quantitativa entre soluções de sistemas diferenciais não autônomos e soluções de sistemas diferenciais promediados, que são autônomos. Aplicamos o averaging à variável independente e os lados direitos dos sistemas estudados são suficientemente pequenos, dependendo de um parâmetro pequeno $\varepsilon$. Além disso, utilizando o Teorema da Função Implícita, o método do averaging garante a existência de soluções periódicas para sistemas periódicos. No trabalho de Buică e Llibre [9], a abordagem do averaging é feita por métodos topológicos e tem como objetivo a resolução de equações com operadores, o que se torna equivalente ao problema de encontrar soluções $T$-periódicas.

O resultado principal desta seção é o Teorema 2.5.1. O primeiro passo na sua demonstração é substituir nosso problema àquele de encontrar zeros de uma função relacionada diretamente ao sistema diferencial dado. Na verdade, temos de estudar a bifurcação dos 
zeros dessa função com respeito ao parâmetro $\varepsilon$ em torno de $\varepsilon=0$. Ao invés do Teorema da Função Implícita, Buică e Llibre utilizaram a teoria do grau de Brouwer.

Aqui, enunciamos o resultado principal de [9] sobre o método do averaging de primeira ordem. Por conveniência, algumas equações que definem o método do averaging serão repetidas a partir do próximo resultado.

Teorema 2.5.1 (Averaging de primeira ordem via grau de Brouwer). Consideremos o seguinte sistema diferencial

$$
\dot{x}=\varepsilon f^{1}(x, t)+\varepsilon^{2} f^{[2]}(x, t, \varepsilon)
$$

onde $f^{1}: D \times \mathbb{R} \rightarrow \mathbb{R}^{n}, f^{[2]}: D \times \mathbb{R} \times\left(-\varepsilon_{f}, \varepsilon_{f}\right) \rightarrow \mathbb{R}^{n}$ são funções contínuas, T-periódicas em $t$ e $D$ é um subconjunto aberto de $\mathbb{R}^{n}$. Definimos $\bar{f}^{1}: D \rightarrow \mathbb{R}^{n}$ como

$$
\bar{f}^{1}(z)=\frac{1}{T} \int_{0}^{T} f^{1}(z, s) d s
$$

e assumamos que:

(i) $f^{1}$ e $f^{[2]}$ são localmente Lipschitz com respeito a $x$;

(ii) para $a \in D \operatorname{com} \bar{f}^{1}(a)=0$, existe uma vizinhança $G$ de a tal que $\bar{f}^{1}(z) \neq 0$ para todo $z \in \bar{G} \backslash\{a\}$ e $d_{B}\left(\bar{f}^{1}, G, 0\right) \neq 0$.

Então, para $|\varepsilon|>0$ suficientemente pequeno, existe uma solução T-periódica $\varphi(\cdot, \varepsilon)$ do sistema (2.5.1) tal que $\varphi(\cdot, \varepsilon) \rightarrow$ a quando $\varepsilon \rightarrow 0$.

O Teorema 2.5.1 tem hipóteses mais fracas quando comparadas a um resultado análogo de Verhulst [45] (Teorema 11.5, p. 168), onde ao invés de (i) é assumido que

(j) $f^{1}, f^{[2]}, \mathrm{D}_{x} f^{1}, \mathrm{D}_{x}^{2} f^{1}$ e $\mathrm{D}_{x} f^{[2]}$ são definidas, contínuas e limitadas por uma constante $M$ (independente de $\varepsilon$ ) em $D \times[0, \infty),-\varepsilon_{f}<\varepsilon<\varepsilon_{f}$,

e ao invés de $(i i)$ assumimos que

$(j j)$ para $a \in D \operatorname{com} \bar{f}^{1}(a)=0$ temos $\mathrm{J}_{\bar{f}^{1}}(a) \neq 0$. 
A partir de agora, denotemos por $\mathrm{D}_{x} f$ a matriz jacobiana de $f$ com respeito à variável $x$ e por $\mathrm{D}_{x}^{2} f$ a matriz das derivadas de segunda ordem. Por $\mathrm{J}_{f}(a)$, denotemos o determinante da jacobiana de $f$ calculada em $a$.

Façamos uma preparação para a demonstração deste resultado.

\subsubsection{Teoria do grau em espaço de dimensão finita}

Nesta seção queremos abordar tão intuitivamente quanto possível as propriedades fundamentais do grau topológico clássico assim como foram formuladas por Brouwer em 1912.

Seguindo as ideias de Browder [8], comecemos com algumas convenções na notação. Consideremos aplicações com domínio em um espaço topológico $X$ e com valores em um espaço topológico $Y$. Se $G$ for um subconjunto aberto de $X$, sejam $\bar{G}$ seu fecho em $X$ e $\partial G$ sua fronteira em $X$. Assim, $f: \bar{G} \rightarrow Y$ será o protótipo das aplicações para o qual uma função grau será definida. Se $f: \bar{G}_{0} \rightarrow Y$ for tal aplicação e $G$ um subconjunto aberto de $G_{0}$, então $f_{G}$ denotará a restrição de $f$ a $\bar{G}$.

Teorema 2.5.2. Sejam $X=Y=\mathbb{R}^{n}$ para um dado inteiro positivo $n$. Para subconjuntos abertos e limitados $G$ de $X$, consideremos aplicações contínuas $f: \bar{G} \rightarrow Y$ e pontos $y_{0} \in Y$ tais que $y_{0} \notin f(\partial G)$. Então, para cada tripla $\left(f, G, y_{0}\right)$, corresponde-se um inteiro $d\left(f, G, y_{0}\right)$ tendo as seguintes propriedades:

(i) Se d(f, $\left.G, y_{0}\right) \neq 0$, então $y_{0} \in f(G)$. Se fo for a aplicação identidade de $X$ em $Y$, então, para cada conjunto aberto e limitado $G$ e $y_{0} \in G$, teremos

$$
d\left(f_{0, G}, G, y_{0}\right)=+1
$$

(ii) (Aditividade) Se $f: \bar{G} \rightarrow Y$ for uma aplicação continua com $G$ um subconjunto aberto e limitado em $X$, e $G_{1}$ e $G_{2}$ formarem um par de subconjuntos abertos disjuntos de $G$ tais que

$$
y_{0} \notin f\left(\bar{G} \backslash\left(G_{1} \cup G_{2}\right)\right) \text {, }
$$


então

$$
d\left(f, G, y_{0}\right)=d\left(f_{G_{1}}, G_{1}, y_{0}\right)+d\left(f_{G_{2}}, G_{2}, y_{0}\right) .
$$

(iii) (Invariância por homotopia) Seja $G$ um subconjunto aberto e limitado de $X$ e consideremos uma homotopia continua $\left\{f_{t} ; 0 \leq t \leq 1\right\}$ de aplicações de $\bar{G}$ em $Y$. Seja $\left\{y_{t} ; 0 \leq t \leq 1\right\}$ uma curva continua em $Y$ tal que $y_{t} \notin f_{t}(\partial G)$, para qualquer $t \in[0,1]$. Então, $d\left(f_{t}, G, y_{t}\right)$ é constante em $t \in[0,1]$.

Teorema 2.5.3. A função grau $d\left(f, G, y_{0}\right)$ é unicamente determinada pelas três condições do Teorema 2.5.2.

O Teorema 2.5.2 é uma versão devidamente formalizada das propriedades do grau de Brouwer clássico.

Intuitivamente, essa função grau que é descrita nos Teoremas 2.5.2 e 2.5.3 destina-se a ser uma contagem algébrica do número de soluções $x$ em $G$ para a equação $f(x)=y_{0}$. Falamos de contagem algébrica porque algumas soluções são contadas positivamente e outras, negativamente.

O caso ideal é de uma aplicação $f$ de classe $\mathcal{C}^{1}$ com somente pontos regulares $x$ como soluções da equação $f(x)=y_{0}$, ou seja, em cada solução, $f^{\prime}(x)$ é uma transformação linear não singular do $\mathbb{R}^{n}$. Desta forma, o número de tais soluções torna-se finito, e diremos que são positivas, se $f^{\prime}(x)$ preservar sua orientação, e negativas, se revertê-la. Então, $d\left(f, G, y_{0}\right)$ é igual ao número de soluções positivas menos o número de soluções negativas. Assim definida, a função grau para tais aplicações é certamente um inteiro. Em [8] vemos uma descrição dos fatos de que a função grau é extensível para todas as aplicações e é invariante por homotopia.

Exemplo 2.5.4. Sejam $G=(-1,1) \subset \mathbb{R}$ e a função $f: \bar{G}=[-1,1] \rightarrow \mathbb{R}$ dada por $f(x)=x^{2}$. Analisemos dois casos.

1. Se $y<0$, então $f^{-1}(y)=\varnothing$. Logo, $d(f, G, y)=0$.

2. Se $y>0$ e $y \neq 1$, então $f^{-1}(y)=\{-\sqrt{y}, \sqrt{y}\}$.

Temos, para $x_{1}=\sqrt{y}, f^{\prime}\left(x_{1}\right) \cdot h=2 \sqrt{y} h>0$, para todo $h>0$, e $f^{\prime}\left(x_{1}\right) \cdot h<0$, para todo $h<0$, isto é, $f^{\prime}\left(x_{1}\right)$ preserva a orientação da solução. E, finalmente, para $x_{2}=-\sqrt{y}$, 
$f^{\prime}\left(x_{2}\right) \cdot h=-2 \sqrt{y} h<0$, para todo $h>0$, e $f^{\prime}\left(x_{2}\right) \cdot h>0$, para todo $h<0$, ou seja, $f^{\prime}\left(x_{2}\right)$ reverte a orientação da solução.

Portanto, $d(f, G, 0)=1-1=0$.

Exemplo 2.5.5. Consideremos a função $f: \bar{G} \rightarrow \mathbb{R}$ definida por $f(x)=\operatorname{sen} x$ com $G=\left(0, \frac{5 \pi}{2}\right)$ e $y_{0}=\frac{\sqrt{2}}{2}$.

Vejamos que $\partial G=\left\{0, \frac{5 \pi}{2}\right\}$ e $f(\partial G)=\{0,1\}$. Assim, $y_{0}=\frac{\sqrt{2}}{2} \notin f(\partial G)$. Logo, $d\left(f, G, \frac{\sqrt{2}}{2}\right)$ está bem definido.

Daí,

$$
f^{-1}\left(\frac{\sqrt{2}}{2}\right)=\left\{\frac{\pi}{4}, \frac{3 \pi}{4}, \frac{9 \pi}{4}\right\} .
$$

Temos $f^{\prime}(x): \mathbb{R} \rightarrow \mathbb{R}$ dada por $f^{\prime}(x) \cdot h=(\cos x) \cdot h$.

Logo, $f^{\prime}\left(\frac{\pi}{4}\right)$ e $f^{\prime}\left(\frac{9 \pi}{4}\right)$ preservam a orientação, enquanto que $f^{\prime}\left(\frac{3 \pi}{4}\right)$ reverte-a. Portanto,

$$
d\left(f, G, \frac{\sqrt{2}}{2}\right)=1-1+1=1
$$

\subsubsection{Algumas observações sobre o grau de Brouwer}

Para subconjuntos $G$ abertos e limitados de $\mathbb{R}^{n}$, tais que $G \subset D$ e $0 \notin f(\partial G, \varepsilon)$ para $\operatorname{algum} \varepsilon$, denotamos por $d_{B}(f(\cdot, \varepsilon), G, 0)$ o grau de Brouwer da função $f(\cdot, \varepsilon)$ com respeito ao conjunto $G$ e o ponto 0, como definido na Seção 2.5.1.

Uma das principais propriedades do grau topológico é que, se $d_{B}(f(\cdot, \varepsilon), G, 0) \neq 0$, então a equação

$$
f(z, \varepsilon)=0
$$

terá solução em $G$ (veja novamente a Seção 2.5.1).

O principal resultado desta seção é o seguinte

Lema 2.5.6. Consideremos as funções contínuas $f^{i}: \bar{G} \rightarrow \mathbb{R}^{n}$, para $i=0, \cdots, k$, e $f, g, r: \bar{G} \times\left[-\varepsilon_{0}, \varepsilon_{0}\right] \rightarrow \mathbb{R}^{n}$ dadas por

$$
g(\cdot, \varepsilon)=f^{0}(\cdot)+\varepsilon f^{1}(\cdot)+\varepsilon^{2} f^{2}(\cdot)+\cdots+\varepsilon^{k} f^{k}(\cdot),
$$


$e$

$$
f(\cdot, \varepsilon)=g(\cdot, \varepsilon)+\varepsilon^{k+1} r(\cdot, \varepsilon)
$$

Assumamos que

$$
g(z, \varepsilon) \neq 0, \quad \text { para todo } z \in \partial G, \varepsilon \in\left[-\varepsilon_{0}, \varepsilon_{0}\right] \backslash\{0\} .
$$

Então, para $|\varepsilon|>0$ suficientemente pequeno, $d_{B}(f(\cdot, \varepsilon), G, 0)$ está bem definido e

$$
d_{B}(f(\cdot, \varepsilon), G, 0)=d_{B}(g(\cdot, \varepsilon), G, 0) .
$$

Demonstração: Usaremos a propriedade da invariância por homotopia do grau de Brouwer. Para cada $\varepsilon \in\left[-\varepsilon_{0}, \varepsilon_{0}\right] \backslash\{0\}$, consideremos a homotopia contínua

$$
h_{t}(\cdot, \varepsilon)=g(\cdot, \varepsilon)+t(f(\cdot, \varepsilon)-g(\cdot, \varepsilon)), \text { para } 0 \leq t \leq 1 .
$$

O que temos que provar é que, quando $\varepsilon$ for suficientemente pequeno, $0 \notin h_{t}(\partial G, \varepsilon)$ para todo $0 \leq t \leq 1$. Suponhamos, por contradição, que, para algum $t_{0} \in(0,1]$ e algum $x_{0} \in \partial G$, tenhamos $h_{t_{0}}\left(x_{0}, \varepsilon\right)=0$. Sendo $r$ uma função contínua em um compacto, existe $M>0$ tal que $|r(z, \varepsilon)| \leq M$, para cada $z \in \bar{G}$ e cada $\varepsilon \in\left(0, \varepsilon_{0}\right)$.

Então, $h_{t_{0}}\left(x_{0}, \varepsilon\right)=0$ implica que

$$
\begin{gathered}
g\left(x_{0}, \varepsilon\right)+t_{0}\left(f\left(x_{0}, \varepsilon\right)-g\left(x_{0}, \varepsilon\right)\right)=0 \Rightarrow \\
\Rightarrow g\left(x_{0}, \varepsilon\right)+t_{0}\left(\varepsilon^{k+1} r\left(x_{0}, \varepsilon\right)\right)=0 \Rightarrow g\left(x_{0}, \varepsilon\right)=-t_{0}\left(\varepsilon^{k+1} r\left(x_{0}, \varepsilon\right)\right) \Rightarrow \\
\Rightarrow\left|g\left(x_{0}, \varepsilon\right)\right| \leq \varepsilon^{k+1}\left|r\left(x_{0}, \varepsilon\right)\right| \Rightarrow\left|g\left(x_{0}, \varepsilon\right)\right| \leq M \varepsilon^{k+1},
\end{gathered}
$$

o que não ocorre para $\varepsilon$ suficientemente pequeno, pois

$$
\left|g\left(x_{0}, \varepsilon\right)\right|=\left|f^{0}\left(x_{0}\right)+\varepsilon f^{1}\left(x_{0}\right)+\varepsilon^{2} f^{2}\left(x_{0}\right)+\cdots+\varepsilon^{k} f^{k}\left(x_{0}\right)\right| \neq 0 .
$$


Sejam $f: D \rightarrow \mathbb{R}^{n}$ uma função de classe $\mathcal{C}^{1}, \operatorname{com} \bar{G} \subset D$, e $Z_{f}=\{z \in G ; f(z)=0\}$. Assumimos também que

$$
\mathrm{J}_{f}(z) \neq 0, \text { para todo } z \in Z_{f}
$$

onde $\mathrm{J}_{f}(z)$ é o determinante da jacobiana de $f$ em $z$. Dessa forma, obtemos que $Z_{f}$ é finito. Então,

$$
d_{B}(f, G, 0)=\sum_{z \in Z_{f}} \operatorname{sign}\left(\mathrm{J}_{f}(z)\right)
$$

onde $n \mapsto \operatorname{sign} n$ é a função sinal.

Observação 2.5.7. Seja $g: D \rightarrow \mathbb{R}^{n}$ uma função de classe $\mathcal{C}^{1}$, com $g(a)=0$, onde $D$ é um subconjunto aberto de $\mathbb{R}^{n}$ e $a \in D$. Segue do Teorema da Função Implícita que, quando $\mathrm{J}_{g}(a) \neq 0$, existirá uma vizinhança $G$ de $a$ tal que $g(z) \neq 0$, para todo $z \in \bar{G} \backslash\{a\}$. Então, $d_{B}(g, G, 0) \in\{-1,1\}$.

Exemplo 2.5.8. O grau de Brouwer da função $f_{0}(z)=z^{2}$ é 0 em qualquer vizinhança da origem. De fato, a função $f_{0}$ tem um único zero, a saber $z_{0}=0$, e temos $f_{0}^{\prime}(0)=0$. Para calcularmos seu grau consideremos $\lambda>0$ dado arbitrariamente, o intervalo $G=(-2 \lambda, 2 \lambda)$ e a função $g(z)=z^{2}-\lambda^{2}$. Dessa forma, $g$ tem dois zeros em $G$ : $-\lambda$ e $\lambda$. O sinal da matriz jacobiana de $g$ é negativo em $-\lambda$ e positivo em $\lambda$. Portanto, $d_{B}(g, G, 0)=0$ e, pelo Lema 2.5.6, $d_{B}\left(f_{0}, G, 0\right)=0$.

O objetivo de Buică e Llibre em [9] é descrever um método no qual se utilize o Lema 2.5.6 a fim de obter respostas ao problema principal de encontrar zeros de uma função conveniente $f: D \times\left(-\varepsilon_{f}, \varepsilon_{f}\right) \rightarrow \mathbb{R}^{n}$.

Assumindo que $D$ seja um subconjunto aberto de $\mathbb{R}^{n}$ e $f$ seja da forma (2.5.5) com $g$ dada por (2.5.4) e $r: D \times\left(-\varepsilon_{f}, \varepsilon_{f}\right) \rightarrow \mathbb{R}^{n}$ contínua. O primeiro passo é encontrar todos os zeros de $f^{0}$. Dado $a \in D$ tal que $f^{0}(a)=0$, se existir uma vizinhança $G$ de $a$ tal que $d_{B}\left(f^{0}, G, 0\right) \neq 0$, então, para $|\varepsilon|>0$ suficientemente pequeno, $f(\cdot, \varepsilon)$ possuirá pelo menos um zero em $G$.

Entretanto, se o grau de Brouwer de $f^{0}$ for zero em pequenas vizinhanças de $a$, ou se não puder ser calculado (o que inclui a possibilidade de $f^{0}$ ser identicamente nula), prosseguiremos com o estudo de $f^{0}+\varepsilon f^{1}$ em alguma vizinhança pequena de $a$ e para $\varepsilon$ 
suficientemente pequeno.

Primeiramente, suponhamos que exista $a_{1 \varepsilon}$ que seja um zero de $f^{0}+\varepsilon f^{1}$ e um subconjunto aberto e limitado $G$ tais que $a_{1 \varepsilon} \in G$ para cada $\varepsilon \neq 0$ suficientemente pequeno $\mathrm{e}$

$$
d_{B}\left(f^{0}+\varepsilon f^{1}, G, 0\right) \neq 0
$$

Assim, pelo Lema 2.5.6, $f(\cdot, \varepsilon)$ possui pelo menos um zero em $G$. Notemos que há a possibilidade de existir outros zeros de $f^{0}+\varepsilon f^{1}$ na mesma vizinhança de $a$, além de $a_{1 \varepsilon}$.

No caso de (2.5.7) não for satisfeita, continuamos com um estudo análogo para a função $f^{0}+\varepsilon f^{1}+\varepsilon^{2} f^{2}$, e assim por diante.

Exemplo 2.5.9. Consideremos a função $f: \mathbb{R}^{2} \rightarrow \mathbb{R}$ dada por

$$
f(z, \varepsilon)=z^{2}-\varepsilon^{2}+\varepsilon^{3} r(z, \varepsilon)
$$

Vamos estimar seu número de zeros. Usando a notação do Lema 2.5.6, temos $f^{0}(z)=z^{2}$, $f^{1}(z)=0$ e $f^{2}(z)=-1$.

Em qualquer vizinhança de 0, o grau de $f^{0}$ é 0 (como visto no Exemplo 2.5.8). Assim, continuamos com o estudo de

$$
\left(f^{0}+\varepsilon f^{1}+\varepsilon^{2} f^{2}\right)(z)=z^{2}-\varepsilon^{2} .
$$

Essa função tem dois zeros: $-\varepsilon$ e $\varepsilon$. Fixemos $\varepsilon_{0}>0$ e consideremos os intervalos abertos $I=\left(0, \varepsilon_{0}\right)$ e $J=\left(-\varepsilon_{0}, 0\right)$. Pela Observação 2.5.7, obtemos que

$$
d_{B}\left(f^{0}+\varepsilon f^{1}+\varepsilon^{2} f^{2}, I, 0\right) \neq 0,
$$

para algum $0<\varepsilon<\varepsilon_{0}$, e a mesma relação vale para $J$ em vez de $I$. Então, pelo Lema 2.5.6, para $\varepsilon>0$ suficientemente pequeno, $f(\cdot, \varepsilon)$ tem no mínimo dois zeros, um em $I$ e outro em $J$.

Vamos supor que $r$ seja de classe $\mathcal{C}^{\infty}$. Usaremos o Teorema da Preparação de Malgrange (veja [12]) para estimar o número de zeros que $f(\cdot, \varepsilon)$ pode admitir. 
Teorema 2.5.10 (Teorema da Preparação de Malgrange). Suponhamos que $U \subset \mathbb{R}^{n} \times \mathbb{R}$ seja um conjunto aberto com $(0,0) \in U$, e $f \in \mathcal{C}^{\infty}(U, \mathbb{R})$ satisfaça

$$
f(x, 0)=x^{k} g(x)
$$

para algum inteiro $k \geq 1$, onde $g$ é suave em uma vizinhança de $x=0$ e $g(0) \neq 0$. Então, existem uma função suave $q$ definida em uma vizinhança $V$ de $(0,0)$ em $\mathbb{R}^{n} \times \mathbb{R}$ e funções $a_{0}(\varepsilon), \cdots, a_{k-1}(\varepsilon)$ de classe $\mathcal{C}^{\infty}$ em uma vizinhança da origem em $\mathbb{R}^{n}$ tais que $q(0,0) \neq 0$, $a_{0}(0)=\cdots=a_{k-1}(0)=0 e$

$$
q(x, \varepsilon) f(x, \varepsilon)=x^{k}+\sum_{i=0}^{k-1} a_{i}(\varepsilon) x^{i}, \quad(x, \varepsilon) \in V
$$

Notemos que a função $f$ é de classe $\mathcal{C}^{\infty}$ e, tomando $g(z)=1$, estamos nas condições do Teorema 2.5.10. Logo, existe uma função $q$ tal que

$$
q(z, \varepsilon) f(z, \varepsilon)=z^{2}+\sum_{i=0}^{1} a_{i}(\varepsilon) z^{i}=z^{2}+a_{1}(\varepsilon) z+a_{0}(\varepsilon)
$$

em uma vizinhança da origem.

Como $q(z, \varepsilon) f(z, \varepsilon)$ é uma função polinomial de segundo grau, ela admite no máximo dois zeros e, portanto, $f$ admite no máximo dois zeros.

Logo, $f(z, \varepsilon)=z^{2}-\varepsilon^{2}+\varepsilon^{3} r(z, \varepsilon)$ possui exatamente dois zeros.

Corolário 2.5.11. Suponhamos que as hipóteses do Lema 2.5.6 estejam satisfeitas para $k=0$ e que, além disso, para $a \in D \operatorname{com} f^{0}(a)=0$, exista uma vizinhança $G$ de a tal que $f^{0}(z) \neq 0$, para todo $z \in \bar{G} \backslash\{a\}$, e $d_{B}\left(f^{0}, G, 0\right) \neq 0$. Então, pelo menos um ramo de zeros bifurca-se de a.

Demonstração: Como $f^{0}(z) \neq 0$, para todo $z \in \bar{G} \backslash\{a\}$, segue da propriedade de excisão do grau de Brouwer (ver [1], p. 40) que $d_{B}\left(f^{0}, G \backslash\{a\}, 0\right)=d_{B}\left(f^{0}, G, 0\right)$. Deduzimos, então, que $d_{B}\left(f^{0}, G_{\mu}, 0\right) \neq 0$, para toda vizinhança $G_{\mu} \subset G$ de $a$. Escolhamos $G_{\mu}$ de forma que $G_{\mu} \rightarrow\{a\}$, quando $\mu \rightarrow 0$.

Logo, para $\varepsilon$ suficientemente pequeno, $f(\cdot, \varepsilon)$ possui no mínimo um zero $a_{\varepsilon} \in G_{\mu}$, e 
podemos escolher $a_{\varepsilon}$ de modo que $a_{\varepsilon} \rightarrow a$, quando $\varepsilon \rightarrow 0$. Além disso, se $J_{f^{0}}(a) \neq 0$, segue do Teorema da Função Implícita que esse ramo de zeros $a_{\varepsilon}$ é único.

Observação 2.5.12. Notemos que no enunciado do Teorema 2.5.1 a única exigência sobre o subconjunto $D \subset \mathbb{R}^{n}$ é que ele seja aberto. Para a definição e propriedades do grau topológico, como feito nas Seções 2.5.1 e 2.5.2, é necessário considerar subconjuntos $G \subset \mathbb{R}^{n}$ abertos e limitados para que a equação $f(x)=0$ possua um número finito de soluções e, assim, ser possível calcular o grau para a função $f$ em $G$. No Teorema 2.5.1 a hipótese mais importante está contida no item $(i i)$ e que se refere à existência de zeros isolados da função promediada. Como é preciso calcular o grau de Brouwer, certamente é necessária alguma limitação dos conjuntos envolvidos, mas essa limitação não necessariamente recai sobre o conjunto $D$. O resultado do teorema está relacionado aos pontos contidos em cada vizinhança dos zeros a da função promediada. Sendo $D$ um aberto, essas vizinhanças podem ser consideradas limitadas, sendo possível o cálculo do grau nesses pontos. O Teorema 2.5.1 é um resultado local.

\subsubsection{Averaging via grau de Brouwer}

Iniciamos esta seção com a justificativa do fato de que o problema de encontrar soluções T-periódicas para um sistema diferencial é equivalente ao de encontrar zeros de uma função correspondente.

Consideremos o sistema diferencial

$$
\dot{x}=f(x, t, \varepsilon),
$$

onde $f: D \times \mathbb{R} \times\left(-\varepsilon_{\bar{f}}, \varepsilon_{\bar{f}}\right) \rightarrow \mathbb{R}^{n}$ é uma função contínua, $T$-periódica em $t$, localmente Lipschitz em $x$ e $D$ um subconjunto aberto de $\mathbb{R}^{n}$. Para cada $z \in D$, denotemos por $x(z, \cdot, \varepsilon):\left[0, t_{z}\right) \rightarrow \mathbb{R}^{n}$ a solução de (2.5.8) com $x(z, 0, \varepsilon)=z$. Assumimos que

$$
t_{z}>T \text {, para todo } z \in D \text {. }
$$


Consideremos, agora, a função $\bar{f}: D \times\left(-\varepsilon_{\bar{f}}, \varepsilon_{\bar{f}}\right) \rightarrow \mathbb{R}^{n}$ dada por

$$
\bar{f}(z, \varepsilon)=\frac{1}{T} \int_{0}^{T} f(x(z, s, \varepsilon), s, \varepsilon) d s .
$$

Toda solução de (2.5.8)

$$
x:[0, T] \rightarrow \mathbb{R}^{n}, \operatorname{com} x(0)=x(T),
$$

pode ser estendida a $\mathbb{R}$ devido a sua periodicidade e, portanto, temos a relação

$$
x(z, T, \varepsilon)-x(z, 0, \varepsilon)=\bar{f}(z, \varepsilon) .
$$

Então, todo par $\left(z_{\varepsilon}, \varepsilon\right)$ tal que

$$
\bar{f}\left(z_{\varepsilon}, \varepsilon\right)=0
$$

produz uma solução periódica $x\left(z_{\varepsilon}, \cdot, \varepsilon\right)$ de $(2.5 .8)$.

A recíproca também é verdadeira, ou seja, para toda solução $T$-periódica de (2.5.8), se denotarmos por $z_{\varepsilon}$ seu valor em $t=0$, então (2.5.12) ficará satisfeita. Consequentemente, o problema de encontrar uma solução T-periódica de (2.5.8) pode ser substituído pelo problema de encontrar zeros da função $\bar{f}(\cdot, \varepsilon)$ dada por (2.5.10).

Para aplicarmos o Lema 2.5.6 precisamos da fórmula de Mac-Laurin de $f$. Quando $f: D \times\left(-\varepsilon_{f}, \varepsilon_{f}\right) \rightarrow \mathbb{R}^{n}$ for contínua e de classe $\mathcal{C}^{k}$ em $\varepsilon$, escrevemos

$$
f(z, \varepsilon)=g(z, \varepsilon)+\varepsilon^{k+1} r(z, \varepsilon)
$$

com $g$ dada por

$$
g(z, \varepsilon)=f(z, 0)+\varepsilon \frac{\partial f}{\partial \varepsilon}(z, 0)+\cdots+\varepsilon^{k} \frac{1}{k !} \frac{\partial^{k} f}{\partial \varepsilon^{k}}(z, 0)
$$

Exceto em $\varepsilon=0$, a função $r$ está bem definida e é contínua. Se provarmos que $r$ é limitada em algum conjunto da forma $K \times\left[-\varepsilon_{0}, \varepsilon_{0}\right]$, com $K$ um subconjunto compacto de $D$, então teremos $r$ contínua em $D \times\left(-\varepsilon_{f}, \varepsilon_{f}\right)$. A continuidade de $r$ é necessária no 
Lema 2.5.6 e, nesse caso, de agora em diante, ao invés de escrevermos a fórmula (2.5.13) com a função $r$ dada explicitamente, usaremos o símbolo de Landau e escreveremos em $K \times\left[-\varepsilon_{0}, \varepsilon_{0}\right]$

$$
f(z, \varepsilon)=g(z, \varepsilon)+\varepsilon^{k+1} \mathcal{O}(1)
$$

Por exemplo, se $\frac{\partial^{k} f}{\partial \varepsilon^{k}}$ for Lipschitz em $K \times\left[-\varepsilon_{0}, \varepsilon_{0}\right]$, então $r$ será limitada nesse conjunto.

Provemos, agora, o Teorema 2.5.1.

Demonstração do Teorema 2.5.1: Para todo $z \in \bar{G}$, existe $\varepsilon_{0}>0$ tal que, quando $\varepsilon \in\left[-\varepsilon_{0}, \varepsilon_{0}\right]$, a solução $x(z, \cdot, \varepsilon)$ está definida em $[0, T]$, ou seja, a relação (2.5.9) é válida. Então, pelo Teorema 2.1.4, existe $t_{z}$ tal que $t_{z}>h_{z}, \operatorname{com} h_{z}=\inf \left(T, \frac{b}{M(\varepsilon)}\right)$, $M(\varepsilon) \geq\left|\varepsilon f^{1}(x, t)+\varepsilon^{2} f^{[2]}(x, t, \varepsilon)\right|$, para todo $t \in[0, T]$, para cada $x$ com $|x-z| \leq b$ e para todo $z \in \bar{G}$. Quando $|\varepsilon|$ for suficientemente pequeno, $M(\varepsilon)$ pode ser arbitrariamente grande, de forma que $h_{z}=T$ para todo $z \in \bar{G}$.

Para todo $t \in[0, T], z \in \bar{G}$ e $\varepsilon \in\left[-\varepsilon_{0}, \varepsilon_{0}\right]$ a seguinte relação está satisfeita

$$
x(z, t, \varepsilon)=z+\varepsilon \int_{0}^{t} f^{1}(x(z, s, \varepsilon), s) d s+\varepsilon^{2} \int_{0}^{t} f^{[2]}(x(z, s, \varepsilon), s, \varepsilon) d s
$$

e a função $\bar{f}$ dada por (2.5.10) torna-se para o nosso sistema

$$
\bar{f}(z, \varepsilon)=\varepsilon \frac{1}{T} \int_{0}^{T} f^{1}(x(z, s, \varepsilon), s) d s+\varepsilon^{2} \frac{1}{T} \int_{0}^{T} f^{[2]}(x(z, s, \varepsilon), s, \varepsilon) d s .
$$

Provemos agora que

$$
f(z, \varepsilon)=\varepsilon \bar{f}^{1}(z)+\varepsilon^{2} \mathcal{O}(1) \text { em } \bar{G} \times\left[-\varepsilon_{0}, \varepsilon_{0}\right]
$$

com $\bar{f}^{1}$ dada por (2.5.2). Notemos que existe $K$ um subconjunto compacto de $D$ tal que $x(z, t, \varepsilon) \in K$ para todo $t \in[0, T], z \in \bar{G}$ e $\varepsilon \in\left[-\varepsilon_{0}, \varepsilon_{0}\right]$.

Pela continuidade de $f^{[2]}$ em $K \times[0, T] \times\left[-\varepsilon_{0}, \varepsilon_{0}\right]$, existe $M_{K}>0$ tal que

$$
f^{[2]}(x(z, s, \varepsilon), s, \varepsilon) \leq M_{K}
$$


ou seja,

$$
\int_{0}^{T} f^{[2]}(x(z, s, \varepsilon), s, \varepsilon) d s \leq \int_{0}^{T} M_{K} d s=M_{K} T=\mathcal{O}(1) .
$$

Por outro lado,

$$
\begin{aligned}
\varepsilon \int_{0}^{T} f^{1}(x(z, s, \varepsilon), s) d s & =\varepsilon \int_{0}^{T}\left[f^{1}(x(z, s, \varepsilon), s)-f^{1}(z, s)+f^{1}(z, s)\right] d s= \\
& =\varepsilon \int_{0}^{T}\left[f^{1}(x(z, s, \varepsilon), s)-f^{1}(z, s)\right] d s+\varepsilon \int_{0}^{T} f^{1}(z, s) d s .
\end{aligned}
$$

Então,

$$
f(z, \varepsilon)-\varepsilon \bar{f}^{1}(z)=\varepsilon \int_{0}^{T}\left[f^{1}(x(z, s, \varepsilon), s)-f^{1}(z, s)\right] d s+\varepsilon^{2} \mathcal{O}(1) .
$$

Como $f^{1}$ é Lipschitz com respeito a $x$ em $[0, T] \times K$ e usando a fórmula (2.5.15), obtemos a seguinte relação

$$
\left|f^{1}(x(z, s, \varepsilon), s)-f^{1}(z, s)\right| \leq L_{K}|x(z, s, \varepsilon)-z| \leq \varepsilon b L_{K}=\varepsilon \mathcal{O}(1)
$$

Portanto, (2.5.16) é satisfeita. Usando o Corolário 2.5.11, obtemos que o item (ii) deste teorema assegura a existência de $z_{\varepsilon}$ tal que $f\left(z_{\varepsilon}, \varepsilon\right)=0$ e $z_{\varepsilon} \rightarrow a$, quando $\varepsilon \rightarrow 0$. Então, $\varphi(\cdot, \varepsilon)=x\left(z_{\varepsilon}, \cdot, \varepsilon\right)$ é uma solução periódica de $(2.5 .1)$ e $\varphi(\cdot, \varepsilon) \rightarrow a$, quando $\varepsilon \rightarrow 0$ (isso é válido pela propriedade de continuidade das soluções de (2.5.1) com relação ao parâmetro e à condição inicial).

Exemplo 2.5.13. [39] Neste exemplo aplicamos o teorema do averaging de primeira ordem a fim de estimar o número máximo de ciclos que podem bifurcar do centro linear perturbado segundo a equação de Liénard:

$$
\left\{\begin{array}{l}
\dot{x}=y \\
\dot{y}=-x-\varepsilon(f(x) y+g(x))
\end{array}\right.
$$

onde $f$ e $g$ são polinômios na variável $x$ com graus 7 e 4, respectivamente. O primeiro passo, neste caso, é colocar o sistema (2.5.18) na forma padrão. Para isto, escolhemos as 
coordenadas polares $(r, \theta)$, onde $x=r \cos \theta$ e $y=r \operatorname{sen} \theta, r>0$. Se escrevermos

$$
f(x)=\sum_{i=0}^{7} a_{i} x^{i} \text { e } g(x)=\sum_{i=0}^{4} b_{i} x^{i}
$$

então o sistema (2.5.18) escreve-se na forma

$$
\left\{\begin{array}{l}
\dot{r}=-\varepsilon\left(\sum_{i=0}^{7} a_{i} r^{i+1} \cos ^{i} \theta \operatorname{sen}^{2} \theta+\sum_{i=0}^{4} b_{i} r^{i} \cos ^{i} \theta \operatorname{sen} \theta\right) \\
\dot{\theta}=-1-\frac{\varepsilon}{r}\left(\sum_{i=0}^{7} a_{i} r^{i+1} \cos ^{i+1} \theta \operatorname{sen} \theta+\sum_{i=0}^{4} b_{i} r^{i} \cos ^{i+1} \theta\right) .
\end{array}\right.
$$

Tomando $\theta$ como a nova variável independente, reescrevemos o sistema (2.5.19) como

$$
\frac{d r}{d \theta}=\varepsilon\left(\sum_{i=0}^{7} a_{i} r^{i+1} \cos ^{i} \theta \operatorname{sen}^{2} \theta+\sum_{i=0}^{4} b_{i} r^{i} \cos ^{i} \theta \operatorname{sen} \theta\right)+\mathcal{O}\left(\varepsilon^{2}\right) .
$$

A correspondente função $\bar{f}^{1}$, dada por $(2.2 .3)$, tem a forma

$$
\bar{f}^{1}(r)=\frac{1}{2 \pi} \int_{0}^{2 \pi}\left(\sum_{i=0}^{7} a_{i} r^{i+1} \cos ^{i} \theta \operatorname{sen}^{2} \theta+\sum_{i=0}^{4} b_{i} r^{i} \cos ^{i} \theta \operatorname{sen} \theta\right) d \theta
$$

Percebendo algumas regularidades nas parcelas de $\bar{f}^{1}(r)$, consideremos as seguintes expressões:

$$
\begin{gathered}
\int_{0}^{2 \pi} \cos ^{2 k+1} \theta \operatorname{sen}^{2} \theta d \theta=0, k=0,1, \ldots, \\
\int_{0}^{2 \pi} \cos ^{2 k} \theta \operatorname{sen}^{2} \theta d \theta=\alpha_{2 k} \neq 0, k=0,1, \ldots, \\
\int_{0}^{2 \pi} \cos ^{k} \theta \operatorname{sen} \theta d \theta=0, k=0,1, \ldots
\end{gathered}
$$

Dessa forma, obtemos

$$
\bar{f}^{1}(r)=\frac{a_{0}}{2} r+\frac{a_{2}}{8} r^{3}+\frac{a_{4}}{16} r^{5}+\frac{5 a_{6}}{128} r^{7}
$$

Observemos que $\bar{f}^{1}(0)=0$ e que $\bar{f}^{1}(r)$ é uma função ímpar. Logo, se $r_{0}$ for raiz de $\bar{f}^{1}$, então $-r_{0}$ também será. Portanto, o polinômio $\bar{f}^{1}(r)$ possui no máximo três raízes 
positivas.

Pelo Teorema 2.5.1, a equação (2.5.18) possui no máximo três ciclos limites.

Os próximos resultados referem-se ao método do averaging de segunda e terceira ordens via grau de Brouwer. Embora suas demonstrações sejam semelhantes à prova do Teorema 2.5.1, estes resultados serão somente enunciados. Sugerimos [9] para uma demonstração detalhada.

Teorema 2.5.14 (Averaging de segunda ordem via grau de Brouwer). Consideremos o seguinte sistema diferencial

$$
\dot{x}(t)=\varepsilon f^{1}(x, t)+\varepsilon^{2} f^{2}(x, t)+\varepsilon^{3} f^{[3]}(x, t, \varepsilon),
$$

onde $f^{1}, f^{2}: D \times \mathbb{R} \rightarrow \mathbb{R}^{n}$ e $f^{[3]}: D \times \mathbb{R} \times\left(-\varepsilon_{f}, \varepsilon_{f}\right) \rightarrow \mathbb{R}^{n}$ são funções contínuas, T-periódicas em $t$ e $D$ um subconjunto aberto de $\mathbb{R}^{n}$. Assumamos que

(i) $f^{1}(\cdot, t) \in \mathcal{C}^{1}(D)$, para todo $t \in \mathbb{R}, f^{1}, f^{2}, f^{[3]}$ e $\mathrm{D}_{x} f^{1}$ sejam localmente Lipschitz com respeito a $x$ e $f^{[3]}$ seja diferenciável com respeito a $\varepsilon$.

Definimos $\bar{f}^{1}, \bar{f}^{2}: D \rightarrow \mathbb{R}^{n}$ por

$$
\begin{gathered}
\bar{f}^{1}(z)=\frac{1}{T} \int_{0}^{T} f^{1}(z, s) d s \\
\bar{f}^{2}(z)=\frac{1}{T} \int_{0}^{T}\left[D_{z} f^{1}(z, s) \cdot \int_{0}^{s} f^{1}(z, t) d t+f^{2}(z, s)\right] d s
\end{gathered}
$$

e assumamos ainda que

(ii) para $G \subset D$ um subconjunto aberto e limitado e para cada $\varepsilon \in\left(-\varepsilon_{f}, \varepsilon_{f}\right) \backslash\{0\}$, exista $a_{\varepsilon} \in G$ tal que $\bar{f}^{1}\left(a_{\varepsilon}\right)+\varepsilon \bar{f}^{2}\left(a_{\varepsilon}\right)=0$ e $d_{B}\left(\bar{f}^{1}+\varepsilon \bar{f}^{2}, G, 0\right) \neq 0$.

Então, para $|\varepsilon|>0$ suficientemente pequeno, existe uma solução T-periódica $\varphi(\cdot, \varepsilon)$ do sistema (2.5.21).

Exemplo 2.5.15. Neste exemplo queremos aplicar a conclusão do Teorema 2.5.14 na 
equação de Van der Pol modificada, dada por

$$
\left\{\begin{array}{l}
\dot{x}=y \\
\dot{y}=-x+\varepsilon x^{2}+\varepsilon^{2}\left(1-x^{2}\right) y
\end{array}\right.
$$

Como no Exemplo 2.4.4, utilizamos a transformação amplitude-fase e o sistema (2.5.23) é reescrito na forma

$$
\left\{\begin{array}{l}
\dot{r}=\varepsilon r^{2} \cos (t-\phi) \operatorname{sen}^{2}(t-\phi)+\varepsilon^{2} r \cos ^{2}(t-\phi)\left[1-r^{2} \operatorname{sen}^{2}(t-\phi)\right] \\
\dot{\phi}=\varepsilon r \operatorname{sen}^{3}(t-\phi)+\varepsilon^{2} \operatorname{sen}(t-\phi) \cos (t-\phi)\left[1-r^{2} \operatorname{sen}^{2}(t-\phi)\right]
\end{array}\right.
$$

Considerando a equação acima escrita na forma $(\dot{r}, \dot{\phi})=\varepsilon f^{1}(r, \phi, t)+\varepsilon^{2} f^{2}(r, \phi, t)$, as funções $\bar{f}^{1}$ e $\bar{f}^{2}$, descritas no Teorema 2.5.14, são expressas por

$$
\bar{f}^{1}(r)=\left(\begin{array}{l}
0 \\
0
\end{array}\right) \text { e } \bar{f}^{2}(r)=\left(\begin{array}{c}
\frac{1}{2}\left(r-\frac{1}{4} r^{3}\right) \\
\frac{5}{12} r^{2}
\end{array}\right)
$$

Dado $G$ um subconjunto aberto e limitado de $\mathbb{R}$ e $\varepsilon \neq 0$, existe $r_{0}=0 \in G$ tal que $\bar{f}^{1}\left(r_{0}\right)+\varepsilon \bar{f}^{2}\left(r_{0}\right)=0$. Além disso, a transformação

$$
\mathrm{D}\left(\bar{f}^{1}+\varepsilon \bar{f}^{2}\right)(0) \cdot\left[h_{1}, h_{2}\right]=\varepsilon\left(\begin{array}{c}
\frac{1}{2} \\
0
\end{array}\right)\left[\begin{array}{ll}
h_{1} & h_{2}
\end{array}\right]=\varepsilon \frac{1}{2} h_{1}
$$

preserva a orientação das soluções e, assim, $d_{B}\left(\bar{f}^{1}+\varepsilon \bar{f}^{2}, G, 0\right)=1 \neq 0$.

Portanto, pelo Teorema 2.5.14, para $|\varepsilon|>0$ suficientemente pequeno, existe uma solução $2 \pi$-periódica de (2.5.23).

Observação 2.5.16. Nos Exemplos 2.4.4 e 2.5.15, nos quais estudamos a equação de Van der Pol modificada, obtemos a mesma conclusão: existe uma única órbita periódica que se bifurca do anel periódico da equação não perturbada. Foram aplicadas as duas abordagens do método do averaging de segunda ordem estudadas nesta dissertação: de caráter analítico no Exemplo 2.4.4 e via grau de Brouwer no Exemplo 2.5.15. Concatenando os resultados de ambos os exemplos, podemos concluir que o problema de perturbação (2.5.23), para $\varepsilon>0$ suficientemente pequeno, possui uma solução $2 \pi$-periódica cuja ex- 
pressão algébrica é dada por

$$
x(t)=2 \cos \left(t-\frac{5}{3} \varepsilon^{2} t\right)+\mathcal{O}(\varepsilon) .
$$

Exemplo 2.5.17. [39] Fazemos novamente referência à equação de Liénard dada por

$$
\left\{\begin{array}{l}
\dot{x}=y \\
\dot{y}=-x-\sum_{k=1}^{2} \varepsilon^{k}\left(f_{n}^{k}(x) y+g_{m}^{k}(x)\right)
\end{array}\right.
$$

onde os polinômios $f_{n}^{k}$ e $g_{m}^{k}$ têm graus $n$ e $m$, respectivamente, com $n, m \geq 1$. O objetivo deste exemplo é destacar os passos que Mereu realizou em [39] ao aplicar o Teorema 2.5.14 para obter o número máximo de ciclos limites do sistema (2.5.24).

Se definirmos

$$
f^{1}(x)=\sum_{i=0}^{n} a_{i} x^{i}, f^{2}(x)=\sum_{i=0}^{n} c_{i} x^{i}, g^{1}(x)=\sum_{i=0}^{m} b_{i} x^{i} \text { e } g^{2}(x)=\sum_{i=0}^{m} d_{i} x^{i}
$$

então o sistema (2.5.24), em coordenadas polares $(r, \theta), r>0$, escreve-se como

$$
\begin{aligned}
\dot{r}= & -\varepsilon\left(\sum_{i=0}^{n} a_{i} r^{i+1} \cos ^{i} \theta \operatorname{sen}^{2} \theta+\sum_{i=0}^{m} b_{i} r^{i} \cos ^{i} \theta \operatorname{sen} \theta\right)- \\
& -\varepsilon^{2}\left(\sum_{i=0}^{n} c_{i} r^{i+1} \cos ^{i} \theta \operatorname{sen}^{2} \theta+\sum_{i=0}^{m} d_{i} r^{i} \cos ^{i} \theta \operatorname{sen} \theta\right), \\
\dot{\theta}= & -1-\frac{\varepsilon}{r}\left(\sum_{i=0}^{n} a_{i} r^{i+1} \cos ^{i+1} \theta \operatorname{sen} \theta+\sum_{i=0}^{m} b_{i} r^{i} \cos ^{i+1} \theta\right)- \\
& -\frac{\varepsilon^{2}}{r}\left(\sum_{i=0}^{n} c_{i} r^{i+1} \cos ^{i+1} \theta \operatorname{sen} \theta+\sum_{i=0}^{m} d_{i} r^{i} \cos ^{i+1} \theta\right) .
\end{aligned}
$$

Tomando $\theta$ como a nova variável independente, o sistema (2.5.25) é escrito como

$$
\frac{d r}{d \theta}=\varepsilon f^{1}(r, \theta)+\varepsilon^{2} f^{2}(r, \theta)+\mathcal{O}\left(\varepsilon^{3}\right)
$$


onde

$$
f^{1}(r, \theta)=\sum_{i=0}^{n} a_{i} r^{i+1} \cos ^{i} \theta \operatorname{sen}^{2} \theta+\sum_{i=0}^{m} b_{i} r^{i} \cos ^{i} \theta \operatorname{sen} \theta
$$

e

$$
\begin{aligned}
f^{2}(r, \theta)= & \left(\sum_{i=0}^{n} c_{i} r^{i+1} \cos ^{i} \theta \operatorname{sen}^{2} \theta+\sum_{i=0}^{m} d_{i} r^{i} \cos ^{i} \theta \operatorname{sen} \theta\right)- \\
& -r \operatorname{sen} \theta \cos \theta\left(\sum_{i=0}^{n} a_{i} r^{i} \cos ^{i} \theta \operatorname{sen} \theta+\sum_{i=0}^{m} b_{i} r^{i-1} \cos ^{i} \theta\right)^{2} .
\end{aligned}
$$

O próximo passo é calcular as expressões das funções $\bar{f}^{1}$ e $\bar{f}^{2}$ definidas em (2.5.22). Obtemos, então, as seguintes fórmulas

$$
\bar{f}^{1}(r)=\frac{1}{2} \sum_{i=0, i \text { par }}^{n} a_{i} \alpha_{i} r^{i+1}
$$

onde $\int_{0}^{2 \pi} \cos ^{2 \delta} \theta \operatorname{sen}^{2} \theta d \theta=\alpha_{2 \delta} \neq 0, \delta=0,1, \ldots, \mathrm{e}$

$$
\begin{aligned}
\bar{f}^{2}(r)= & r\left(\rho_{10} a_{1} b_{0}+\left(\rho_{12} a_{1} b_{2}+\rho_{30} a_{3} b_{0}\right) r^{2}+\left(\rho_{14} a_{1} b_{4}+\rho_{32} a_{3} b_{2}+\rho_{50} a_{5} b_{0}\right) r^{4}+\cdots\right. \\
& \left.\cdots+\rho_{\ell \delta} a_{\ell} b_{\delta} r^{\ell+\delta-1}+A_{0} c_{0}+A_{2} c_{2} r^{2}+\cdots+A_{\beta} c_{\beta} r^{\beta}\right)
\end{aligned}
$$

onde

$\ell$ é o maior número ímpar menor ou igual a $n$;

$\beta$ é o maior número par menor ou igual a $n$;

$\alpha_{i j}$ são constantes que aparecem nos cálculos de $\int_{0}^{\theta} \cos ^{i} \phi \operatorname{sen}^{2} \phi d \phi$;

$\int_{0}^{2 \pi} \cos ^{2 i} \theta \operatorname{sen}^{2} \theta d \theta=A_{2 i} \neq 0, i=0,1, \ldots ;$

$\int_{0}^{2 \pi} \cos ^{2 i} \theta \operatorname{sen} \theta \operatorname{sen}((2 k+1) \theta) d \theta=B_{2 i}^{2 k+1} \neq 0, i, k=0,1, \ldots$;

$\rho_{i j}=\tilde{\alpha}_{i j}+2 A_{i+j+1}, i, j=0,1, \ldots ; \mathrm{e}$

$\tilde{\alpha}=-\frac{1+i}{j+i} A_{i+j+1}+j\left(\alpha_{1 i} B_{j}^{1}+\alpha_{2 i} B_{j}^{2}+\cdots+\alpha_{\frac{i+3}{2} i} B_{j}^{i+2}\right), i, j=0,1, \ldots$

O polinômio $\bar{f}^{1}$ tem até $\left[\frac{n}{2}\right]$ (parte inteira de $\frac{n}{2}$ ) raízes positivas e será identicamente nulo se, e somente se, $a_{i}=0$ para todo $i$ par. Para encontrar as raízes positivas de $\bar{f}^{2}$ devemos encontrar os zeros de um polinômio na variável $r^{2}$ de grau igual ao $\max \left\{\frac{\ell+\delta-1}{2}, \frac{b}{2}\right\}$. Sabendo que $\frac{b}{2}=\left[\frac{n}{2}\right]$ e $\frac{\ell+\delta-1}{2}=\left[\frac{n-1}{2}\right]+\left[\frac{m}{2}\right]$, segue que $\bar{f}^{2}$ tem até $\max \left\{\left[\frac{n-1}{2}\right]+\left[\frac{m}{2}\right],\left[\frac{n}{2}\right]\right\}$ 
raízes positivas. Além disso, podemos escolher os coeficientes $a_{i}, b_{j}$ e $c_{\delta}$ de tal modo que $\bar{f}^{2}$ tenha exatamente $\max \left\{\left[\frac{n-1}{2}\right]+\left[\frac{m}{2}\right],\left[\frac{n}{2}\right]\right\}$ raízes positivas.

Todos os detalhes desta demonstração podem ser encontrados em [39], pp. 24-27.

Observação 2.5.18. O Teorema 2.5.14 tem hipóteses mais fracas quando comparadas ao resultado análogo apresentado em [33] (Corolário 6) por Llibre, ou em [22] (Teorema 2.2) por Hartono e van der Burgh, onde $D$ é um domínio limitado de $\mathbb{R}^{n}$ e ao invés de $(i)$ é assumido que

(j) $f^{1}, f^{2}, f^{[3]}, \mathrm{D}_{x} f^{1}, \mathrm{D}_{x}^{2} f^{1}, \mathrm{D}_{x} f^{2}, \mathrm{D}_{x} f^{[3]}$ são definidas, contínuas e limitadas em $D \times[0, \infty) \times\left(-\varepsilon_{f}, \varepsilon_{f}\right)$,

e ao invés de (ii) assume-se que

$(j j) \bar{f}^{1}(z)=0$, para todo $z \in D$, e para $a \in D \operatorname{com} \bar{f}^{2}(a)=0$, temos $\mathrm{J}_{\bar{f}^{2}}(a) \neq 0$.

Embora seja válido para sistemas de dimensão arbitrária, enunciaremos o resultado do averaging de terceira ordem para $n=1$ a fim de evitar a escrita de fórmulas muito complicadas.

Teorema 2.5.19 (Averaging de terceira ordem via grau de Brouwer). Consideremos o seguinte sistema diferencial

$$
\dot{x}(t)=\varepsilon f^{1}(x, t)+\varepsilon^{2} f^{2}(x, t)+\varepsilon^{3} f^{3}(x, t)+\varepsilon^{4} f^{[4]}(x, t, \varepsilon),
$$

onde $f^{1}, f^{2}, f^{3}: D \times \mathbb{R} \rightarrow \mathbb{R}$ e $f^{[4]}: D \times \mathbb{R} \times\left(-\varepsilon_{f}, \varepsilon_{f}\right) \rightarrow \mathbb{R}$ são funções contínuas, T-periódicas em $t$, e $D$ um intervalo aberto de $\mathbb{R}$. Assumamos que

(i) $f^{1}(\cdot, t) \in \mathcal{C}^{2}(D), f^{2}(\cdot, t) \in \mathcal{C}^{1}(D)$ para todo $t \in \mathbb{R}, f^{1}, f^{2}, f^{3}, f^{[4]}, \mathrm{D}_{x}^{2} f^{1}, \mathrm{D}_{x} f^{2}$ sejam localmente Lipschitz com respeito a $x$ e $f^{[4]}$ seja duas vezes diferenciável com respeito $a \varepsilon$.

Definimos $\bar{f}^{1}, \bar{f}^{2}: D \rightarrow \mathbb{R}$ por $(2.5 .22)$ e $\bar{f}^{3}: D \rightarrow \mathbb{R}$ por

$$
\begin{gathered}
\bar{f}^{3}(z)=\frac{1}{T} \int_{0}^{T}\left[\frac{1}{2} \frac{\partial^{2} f^{1}}{\partial z^{2}}(z, s)\left(y_{1}(z, s)\right)^{2}+\frac{1}{2} \frac{\partial f^{1}}{\partial z}(z, s) y_{2}(z, s)+\right. \\
\left.+\frac{\partial f^{2}}{\partial z}(z, s) y_{1}(z, s)+f^{3}(z, s)\right] d s
\end{gathered}
$$


onde

$$
y_{1}(z, s)=\int_{0}^{s} f^{1}(z, t) d t \quad e \quad y_{2}(z, s)=\int_{0}^{s}\left[\frac{\partial f^{1}}{\partial z}(z, t) \int_{0}^{t} f^{1}(z, r) d r+f^{2}(z, t)\right] d t .
$$

Além disso, assumamos que

(ii) para $G \subset D$ um intervalo aberto e limitado e para cada $\varepsilon \in\left(-\varepsilon_{f}, \varepsilon_{f}\right) \backslash\{0\}$, exista $a_{\varepsilon} \in G$ tal que $\bar{f}^{1}\left(a_{\varepsilon}\right)+\varepsilon \bar{f}^{2}\left(a_{\varepsilon}\right)+\varepsilon^{2} \bar{f}^{3}\left(a_{\varepsilon}\right)=0$ e $d_{B}\left(\bar{f}^{1}+\varepsilon \bar{f}^{2}+\varepsilon^{2} \bar{f}^{3}, G, 0\right) \neq 0$.

Então, para $|\varepsilon|>0$ suficientemente pequeno, existe uma solução T-periódica $\varphi(\cdot, \varepsilon)$ do sistema (2.5.26).

Até este ponto da dissertação estudamos e aplicamos o método das integrais abelianas e o método do averaging, que são utilizados no estudo das bifurcações de ciclos limites em sistemas diferenciais polinomiais de grau $n$. Ambos os métodos são aplicáveis para o caso de dimensão dois, mas apenas o averaging pode ser utilizado para dimensões maiores. Em [39], pp. 45-62, Mereu aplica o método do averaging de primeira ordem para investigar o número de ciclos limites que surgem das órbitas periódicas de um centro em $\mathbb{R}^{4}$ após uma perturbação de uma classe de sistemas diferenciais polinomiais.

Observamos que o método das integrais abelianas é utilizado para um estudo local, já que é necessário considerar uma vizinhança do ponto singular do tipo centro que contenha o anel periódico ao redor da singularidade para que a técnica seja aplicada. Por outro lado, Sanders, Verhulst e Murdock [41] afirmam que a teoria do averaging é utilizada não somente para construir soluções periódicas e estimar seu erro, mas também para provar a existência de órbitas periódicas e determinar sua estabilidade. Além disso, o averaging possibilita estudar propriedades qualitativas mais gerais [33]. Enquanto que usando as integrais abelianas estudamos quais órbitas do sistema não perturbado se tornam ciclos limites do sistema perturbado, através do averaging temos conhecida a configuração global dos ciclos limites obtidos após bifurcação; a cada zero da função promediada temos uma órbita periódica do sistema perturbado e sabemos sua distribuição no plano.

Na próxima seção apresentamos, dentre outros, um resultado de equivalência entre os métodos das integrais abelianas e do averaging topológico para sistemas polinomiais integráveis no plano. 


\subsubsection{Averaging versus integrais abelianas em sistemas integráveis}

Seguindo as ideias de Buică e Llibre [9], nesta seção estudamos a relação entre o método do averaging com a função sucessão para sistemas autônomos e integráveis no plano.

Mais especificamente, consideremos o sistema planar

$$
\left\{\begin{array}{l}
\dot{x}=P(x, y) \\
\dot{y}=Q(x, y)
\end{array}\right.
$$

onde $P, Q: \mathbb{R}^{2} \rightarrow \mathbb{R}$ são funções contínuas.

Consideremos o sistema (2.5.27) sob a seguinte hipótese:

(hip) O sistema (2.5.27) possui um anel periódico em torno do ponto singular $(0,0)$ dado por

$$
\gamma_{h}=\left\{(x, y) \in \mathbb{R}^{2} ; H(x, y)=h, h_{c}<h<h_{s}\right\}
$$

A função $H$ é a integral primeira de (2.5.27), $h_{c}$ é o nível do ponto crítico de $H$ correspondendo ao centro $(0,0)$ e $h_{s}$ denota o valor de $H$ para o qual o anel periódico termina. Sem perda de generalidade, podemos assumir que $h_{s}>h_{c} \geq 0$. Denotamos por $M=M(x, y)$ o fator de integração do sistema (2.5.27) correspondente à integral primeira $H$.

Consideremos, agora, perturbações de (2.5.27) da forma

$$
\left\{\begin{array}{l}
\dot{x}=P(x, y)+\varepsilon p(x, y) \\
\dot{y}=Q(x, y)+\varepsilon q(x, y)
\end{array}\right.
$$

onde $p, q: \mathbb{R}^{2} \rightarrow \mathbb{R}$ são funções contínuas.

Buică e Llibre propuseram uma maneira de aplicar o método do averaging para estudar ciclos limites de (2.5.28), para $\varepsilon$ suficientemente pequeno, que bifurcam em $\varepsilon=0$ de trajetórias do anel periódico de (2.5.27).

O primeiro objetivo é escrever o sistema (2.5.28) na forma padrão para aplicarmos o método do averaging, ou seja, na forma (2.5.1). O sistema diferencial nessa forma padrão 
descreverá a dependência entre a raiz quadrada da energia, $R=\sqrt{h}$, e o ângulo $\varphi$ das coordenadas polares. O campo de vetores dessa equação nas coordenadas $(R, \varphi)$ será $2 \pi$-periódico e suas soluções $2 \pi$-periódicas serão trajetórias periódicas de (2.5.28).

Teorema 2.5.20. Assumamos que (hip) seja válida para o sistema (2.5.27) e que

$$
x Q(x, y)-y P(x, y) \neq 0, \text { para todo }(x, y) \in \gamma_{h} .
$$

Seja $\rho:\left(\sqrt{h_{c}}, \sqrt{h_{s}}\right) \times[0,2 \pi) \rightarrow[0, \infty)$ uma função contínua tal que

$$
H(\rho(R, \varphi) \cos \varphi, \rho(R, \varphi) \operatorname{sen} \varphi)=R^{2}
$$

para todo $R \in\left(\sqrt{h_{c}}, \sqrt{h_{s}}\right)$ e todo $\varphi \in[0,2 \pi)$. Então, a equação diferencial que descreve a dependência entre a raiz quadrada da energia, $R=\sqrt{h}$, e o ângulo $\varphi$ para o sistema (2.5.28) é dada por

$$
\frac{d R}{d \varphi}=\varepsilon \frac{M\left(x^{2}+y^{2}\right)(Q p-P q)}{2 R(Q x-P y)+2 R \varepsilon(q x-p y)}
$$

onde $x=\rho(R, \varphi) \cos \varphi$ e $y=\rho(R, \varphi) \operatorname{sen} \varphi$.

Tomemos $\varepsilon_{f}>0$ suficientemente pequeno e $D=\bigcup_{h_{c^{*}}<h<h_{s^{*}}} \gamma_{h}$, onde $h_{c}<h_{c^{*}}<h_{s^{*}}<$ $<h_{s}$ são fixados arbitrariamente próximos de $h_{c}$ e $h_{s}$, respectivamente. O campo de vetores da equação (2.5.31) é bem definido e contínuo em $D \times\left(-\varepsilon_{f}, \varepsilon_{f}\right)$ e é $2 \pi$-periódico com respeito a $\varphi$.

Demonstração: Consideremos as seguintes relações:

$$
\frac{\partial H}{\partial x} P+\frac{\partial H}{\partial y} Q=0, \frac{\partial H}{\partial y}=-M P \text { e } \frac{\partial H}{\partial x}=M Q
$$

que são válidas no anel periódico, já que $H$ é uma integral primeira e $M$ é um fator integrante de (2.5.27). Definimos a função

$$
G(r, R, \varphi)=H(r \cos \varphi, r \operatorname{sen} \varphi)-R^{2}
$$

em cada ponto $(r, \varphi)$ do anel periódico (que é um conjunto aberto) e para cada 
$R \in\left(\sqrt{h_{c}}, \sqrt{h_{s}}\right)$. Aqui, $(r, \varphi)$ denota as coordenadas polares. Assim, temos

$$
\frac{\partial G}{\partial r}=\frac{\partial H}{\partial x} \cos \varphi+\frac{\partial H}{\partial y} \operatorname{sen} \varphi=\frac{M(x, y)}{r}(Q(x, y) x-P(x, y) y)
$$

onde $x=r \cos \varphi$ e $y=r \operatorname{sen} \varphi$. Para cada $\left(r_{0}, \varphi_{0}\right)$ no anel periódico, existe um $R_{0}$ tal que $G\left(r_{0}, R_{0}, \varphi_{0}\right)=0$. A hipótese (2.5.29) assegura que $\frac{\partial G}{\partial r}\left(r_{0}, R_{0}, \varphi_{0}\right) \neq 0$. Pelo Teorema da Função Implícita, em torno de todo ponto $\left(R_{0}, \varphi_{0}\right)$, existe uma única função contínua $\rho=\rho(R, \varphi)$ tal que (2.5.30) seja verdadeira. Consequentemente, essa função $\rho=\rho(R, \varphi)$ é bem definida em todo domínio $\left(\sqrt{h_{c}}, \sqrt{h_{s}}\right) \times[0,2 \pi)$ e satisfaz $(2.5 .30)$.

A dependência entre a raiz quadrada da energia e o tempo é dada por $R(t)=\sqrt{H(x(t), y(t))}$, e entre o ângulo $\varphi$ e o tempo é $\varphi(t)=\arctan \frac{y(t)}{x(t)}$, quando $(x(t), y(t)) \in \gamma_{h}, t \in \mathbb{R}$. Logo, obtemos

$$
\begin{aligned}
\dot{R} & =\frac{1}{2} \frac{1}{\sqrt{H(x(t), y(t))}} \frac{d}{d t} H(x(t), y(t))=\frac{1}{2 R(t)}\left[\frac{\partial H}{\partial x} \dot{x}+\frac{\partial H}{\partial y} \dot{y}\right]= \\
& =\frac{1}{2 R}[M Q(P+\varepsilon p)-M P(Q+\varepsilon q)]=\varepsilon \frac{M(Q p-P q)}{2 R}
\end{aligned}
$$

e

$$
\begin{aligned}
\dot{\varphi} & =\frac{1}{1+\left(\frac{y}{x}\right)^{2}} \frac{d}{d t}\left(\frac{y(t)}{x(t)}\right)=\frac{x^{2}}{x^{2}+y^{2}}\left[\frac{\dot{y} x-y \dot{x}}{x^{2}}\right]= \\
& =\frac{1}{x^{2}+y^{2}}[(Q+\varepsilon q) x-(P+\varepsilon p) y]=\frac{(Q x-P y)+\varepsilon(q x-p y)}{x^{2}+y^{2}} .
\end{aligned}
$$

Eliminando o tempo das duas equações acima, obtemos a equação (2.5.31).

A condição (2.5.29) implica que o campo vetorial (2.5.31) esteja bem definido em $D \times\left(-\varepsilon_{f}, \varepsilon_{f}\right)$, para $\varepsilon_{f}$ suficientemente pequeno. Além disso, o campo é contínuo e $2 \pi$-periódico em $\varphi$.

Um resultado importante é o que se segue, o qual afirma que a aplicação do método do averaging para sistemas planares nas condições desta seção é equivalente ao estudo da função sucessão. Em particular, o método do averaging de primeira ordem é equivalente ao estudo da função de Melnikov (integral abeliana) de primeira ordem. Buică e Llibre [9] dão referências para mais detalhes nesta direção. 
Teorema 2.5.21. A função $\bar{f}:\left(\sqrt{h_{c^{*}}}, \sqrt{h_{s^{*}}}\right) \times\left(-\varepsilon_{f}, \varepsilon_{f}\right) \rightarrow \mathbb{R}$ descrita por (2.5.10) para a equação (2.5.31) é dada por

$$
\bar{f}(R, \varepsilon)=\varepsilon \frac{1}{2 \pi} \int_{0}^{2 \pi} \frac{M\left(x^{2}+y^{2}\right)(Q p-P q)}{2 R(Q x-P y)+2 R \varepsilon(q x-p y)} d \varphi
$$

e a função $\bar{f}^{1}:\left(\sqrt{h_{c^{*}}}, \sqrt{h_{s^{*}}}\right) \rightarrow \mathbb{R}$ descrita por (2.5.2) para a equação (2.5.30) é dada por

$$
\bar{f}^{1}(R)=\frac{1}{2 \pi} \int_{0}^{2 \pi} \frac{M\left(x^{2}+y^{2}\right)(Q p-P q)}{2 R(Q x-P y)} d \varphi
$$

onde $M=M(x, y)$ é o fator de integração do sistema (2.5.27) correspondente à integral primeira $H$, e $x=\rho(R, \varphi) \cos \varphi$ e $y=\rho(R, \varphi) \operatorname{sen} \varphi$.

Além disso, a função (2.5.32) é a função sucessão e (2.5.33) é a correspondente função de Melnikov (integral abeliana) de primeira ordem do sistema (2.5.28).

Demonstração: Escrevendo (2.5.31) utilizando a fórmula de Mac-Laurin de $f$ com respeito a $\varepsilon$, obtemos

$$
\frac{d R}{d \varphi}=\varepsilon f^{1}(R, \varphi)+\varepsilon^{2} f^{[2]}(R, \varphi, \varepsilon),
$$

onde $f^{1}(R, \varphi)=\frac{M\left(x^{2}+y^{2}\right)(Q p-P q)}{2 R(Q x-P y)}$ e $f^{[2]}$ é o resto de Lagrange.

As equações (2.5.32) e (2.5.33) são imediatas. Resta-nos mostrar que o número de zeros da integral abeliana de primeira ordem e da função promediada de primeira ordem são os mesmos.

Seguindo a notação de [38], consideremos o sistema original na forma padrão

$$
\dot{x}=\varepsilon f^{1}(x, t)+\varepsilon^{2} f^{[2]}(x, t, \varepsilon), \quad x(0)=x_{0},
$$

onde as funções $f^{1}$ e $f^{[2]}$ são analíticas e $T$-periódicas em $t$. Essa transformação é feita usando coordenadas polares, ou escrevendo o sistema como a equação (2.5.31) que descreve a dependência entre o ângulo e a raiz quadrada da energia. Denotemos por $x(t, \varepsilon)$ a solução de (2.5.34), e expandindo essa solução em série de potências em $\varepsilon$, obtemos

$$
x(t, \varepsilon)=x_{0}+\varepsilon x_{1}(t)+\varepsilon^{2} x_{2}(t)+\cdots .
$$


A condição inicial $x(0)=x_{0}$ implica que $x_{i}(0)=0, i \geq 1$. Notemos que

$$
x(T, \varepsilon)-x_{0}
$$

é o primeiro termo em $\varepsilon$ da função sucessão e $x_{i}(T)$ é a integral abeliana de ordem $i \geq 1$. Substituímos a solução $x(t, \varepsilon)$ em (2.5.34) e obtemos

$$
\varepsilon \dot{x}_{1}+\mathcal{O}\left(\varepsilon^{2}\right)=\varepsilon f^{1}\left(x_{0}, t\right)+\mathcal{O}\left(\varepsilon^{2}\right)
$$

Até os termos de $\mathcal{O}\left(\varepsilon^{2}\right)$, temos $\dot{x}_{1}=f^{1}\left(x_{0}, t\right)$, da qual obtemos a relação

$$
\int_{0}^{T} \dot{x}_{1}(s) d s=\int_{0}^{T} f^{1}\left(x_{0}, s\right) d s
$$

Sabendo que $x_{1}(0)=0$, segue-se que

$$
x_{1}(T)=\int_{0}^{T} f^{1}\left(x_{0}, s\right) d s=: \bar{f}^{1}\left(x_{0}\right) .
$$

Desta equação, segue que o número de zeros da função promediada de primeira ordem $\bar{f}^{1}\left(x_{0}\right)$ corresponde ao número de zeros da função sucessão e, consequentemente, com o número de zeros da primeira função de Melnikov, que corresponde com a integral abeliana do sistema perturbado no anel periódico em torno da origem.

Exemplo 2.5.22. [18] Consideremos a equação de Van der Pol dada por

$$
\left\{\begin{array}{l}
\dot{x}=y \\
\dot{y}=-x+\varepsilon\left(1-x^{2}\right) y .
\end{array}\right.
$$

Nosso objetivo neste exemplo é obter os mesmos resultados descritos nos Exemplos 1.4.10 e 2.3.5 utilizando o método apresentado na Seção 2.5.4. Para isso, identificamos:

$$
P(x, y)=y, Q(x, y)=-x, p(x, y)=0 \text { e } q(x, y)=\left(1-x^{2}\right) y
$$

Desta forma, a função hamiltoniana relacionada à equação não perturbada é expressa 
por

$$
H(x, y)=\frac{x^{2}}{2}+\frac{y^{2}}{2}
$$

e seu fator de integração é constante e igual a $M(x, y)=1$. O anel periódico em torno da origem é dado por

$$
\gamma_{h}=\left\{(x, y) \in \mathbb{R}^{2} ; H(x, y)=h, h \in(0, \infty)\right\}
$$

Observemos que

$$
x Q(x, y)-y P(x, y)=-x^{2}-y^{2} \neq 0, \text { para todo }(x, y) \in \gamma_{h} .
$$

Resolvendo a equação $H(\rho \cos \varphi, \rho \operatorname{sen} \varphi)=R^{2}$ na variável $\rho$, obtemos

$$
\rho=\rho(R, \varphi)=\sqrt{2} R
$$

$\operatorname{com} R>0$ e $\varphi \in[0,2 \pi)$.

Logo, pelo Teorema 2.5.20, a equação (2.5.31) correspondente é escrita como

$$
\frac{d R}{d \varphi}=\varepsilon R\left(1-2 R^{2} \cos ^{2} \varphi\right) \operatorname{sen}^{2} \varphi+\varepsilon^{2} R \cos \varphi\left(1-2 R^{2} \cos ^{2} \varphi\right)^{2} \operatorname{sen}^{3} \varphi+\mathcal{O}\left(\varepsilon^{3}\right)
$$

Chamando

$$
f^{1}(R, \varphi)=R\left(1-2 R^{2} \cos ^{2} \varphi\right) \operatorname{sen}^{2} \varphi \text { e } f^{2}(R, \varphi)=R \cos \varphi\left(1-2 R^{2} \cos ^{2} \varphi\right)^{2} \operatorname{sen}^{3} \varphi
$$

e aplicando o método do averaging à equação anterior, a função promediada tem sua expressão dada por

$$
\bar{f}^{1}(R)=\frac{1}{2}\left(R-\frac{R^{3}}{2}\right),
$$

cujos zeros são $-\sqrt{2}, 0$ e $\sqrt{2}$. Como $R_{0}=0$ indica a origem do sistema, consideremos $R_{1}=\sqrt{2}$, que é a única raiz positiva de $\bar{f}^{1}$. Como $\mathrm{D} \bar{f}^{1}(R)=\frac{1}{2}\left(1-\frac{3}{2} R^{2}\right) \neq 0$, segue do Teorema da Função Implícita, existe uma vizinhança $V$ de $R_{1}=\sqrt{2} \mathrm{em} \mathbb{R}$ tal que $\bar{f}^{1}(R) \neq 0$, para todo $R \in V \backslash\{\sqrt{2}\}$. Além disso, $\mathrm{D} \bar{f}^{1}(\sqrt{2}) \cdot h=-h, h \in \mathbb{R}$, reverte a 
orientação das soluções. Dessa forma, $d_{B}\left(\bar{f}^{1}, V, 0\right)=-1 \neq 0$.

Portanto, pelo Teorema 2.5.1, para $|\varepsilon|>0$ suficientemente pequeno, existe uma solução $2 \pi$-periódica do sistema (2.5.35) que tende à circunferência de raio $\sqrt{2}$ quando $\varepsilon \rightarrow 0$. 


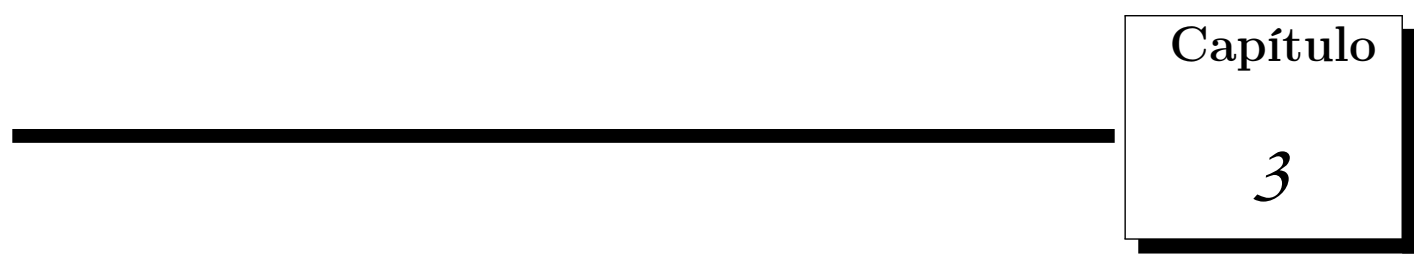

\section{Considerações finais}

Esta dissertação foi motivada pelo conhecido XVI problema de Hilbert. Este problema, proposto em 1900, que questiona sobre o número máximo e a posição de ciclos limites em sistemas diferenciais polinomiais no plano, vem intrigando e desafiando a comunidade científica, principalmente os matemáticos. Com o passar do tempo, várias outras versões do problema foram enunciadas e, em 1977, Arnol'd propôs o que ficou conhecida como a versão fraca do XVI problema de Hilbert. O objetivo principal do problema de Arnol'd é de investigar o número máximo de ciclos limites que bifurcam de centros em sistemas diferenciais polinomiais no plano. Nesta dissertação o objetivo foi destacar a importância do problema proposto e enfatizar a utilização de dois métodos que nos permitissem estimar o número máximo de zeros de ciclos limites em tais sistemas.

Para cumprir o objetivo desta dissertação estudamos e analisamos vários livros e artigos de pesquisadores da área que foram citados ao longo do texto e encontram-se descritos nas referências bibliográficas deste trabalho. Vale ressaltar que foram realizadas algumas visitas de campo com o intuito de nos reunirmos com profissionais mais experientes, como Claudio A. Buzzi (IBILCE/Unesp) e Joan C. Artés (UAB/Barcelona), que visitou o ICMC/USP durante o mês de julho de 2010. Além disso, participamos de congressos e workshops onde pudemos divulgar nossa pesquisa e, ao mesmo tempo, entrar em contato com outros pesquisadores de áreas afins.

O método das integrais abelianas, exposto no Capítulo 1, foi basicamente estudado 
a partir da referência [13], uma publicação do Centro de Pesquisas Matemáticas de Barcelona, e o conteúdo foi complementado pelas referências citadas no mesmo. Fizemos uma abordagem do método das equações de Picard-Fuchs, utilizado para estimar do número de zeros da razão de duas integrais abelianas.

Ainda sobre o método das integrais abelianas, selecionamos um sistema diferencial polinomial, denominado $P_{23}$, que tem uma singularidade do tipo centro. Seguindo as mesmas ideias descritas em [47] (onde os sistemas estudados são mais genéricos do que o $P_{23}$ ), fizemos uma tentativa de estimar o número máximo de ciclos limites para esse sistema. Contudo, não obtivemos resultados satisfatórios para o nosso objetivo. O que destacamos desse estudo foi a possibilidade de aproximação e interação com Artés, que nos ensinou técnicas de manuseio com os softwares Maple e Mathematica.

No Capítulo 2 descrevemos o método do averaging. Analisamos duas abordagens do método: uma de caráter analítico e outra, topológico. Para o primeiro caso, seguimos as ideias contidas em $[41,40]$ e referências ali citadas. Destacamos, também, a importância de uma equação diferencial estar na forma padrão para que o método do averaging seja aplicado e propomos algumas técnicas para que tal forma seja obtida. Em relação ao averaging topológico, em [9] faz-se uma abordagem do método via grau de Brouwer. Para sua compreensão, foi feita uma descrição da teoria do grau elaborada por Brouwer em 1912 e, em seguida, um estudo da técnica apresentada em [9]. Na última seção do capítulo expomos a relação existente entre o método das integrais abelianas com o método do averaging para sistemas diferenciais integráveis.

Cabe ressaltar que pretendemos avançar com o estudo do sistema $P_{23}$. Recentemente, deparamo-nos com o artigo [31] onde Li e Llibre estudam um sistema semelhante ao $P_{23}$ pelo método envolvendo a equação de Picard-Fuchs. Nossa intenção é seguir os mesmos passos e buscar uma estimativa para o número de ciclos limites do sistema $P_{23}$ com uma pertubação polinomial quadrática. Dessa forma, estaremos contribuindo com o estudo de um sistema particular dentro do XVI problema de Hilbert. 


\section{Referências Bibliográficas}

[1] O. B. Almeida. Teoria do grau e aplicações. Dissertação (Mestrado em Matemática) - CCT/UFCG, Campina Grande, (2006).

[2] V. I. Arnol'd. Loss of stability of self-oscillations close to resonance and versal deformations of equivariant vector fields, Funct. Anal. Appl. 11 (1977), 85-92.

[3] V. I. Arnol'd. Mathematical methods of classical mechanics. Graduate Texts in Mathematics, 60. New York: Springer-Verlag, 1989.

[4] V. I. Arnol'd. Ten problems, Adv. Soviet. Math. 1 (1990), 1-8.

[5] J. C. Artés; J. Llibre; N. Vulpe. Quadratic systems with a rational first integral of degree three: a complete classification in the coefficient space $\mathbb{R}^{12}$, Preprint (2009).

[6] J. G. Besjes. On the assymptotic methods for non-linear differential equations, Journal de Mécanique 8 (1969), 357-372.

[7] T. R. Blows; L. M. Perko. Bifurcation of limit cycles from centers and separatrix cycles of planar analytic systems, SIAM Rev. 36 (1994), 341-376.

[8] F. Browder. Fixed point theory and nonlinear problems, Bull. Amer. Math. Soc. 9 (1983), 1-39.

[9] A. Buică; J. Llibre. Averaging methods for finding periodic orbits via Brouwer degree, Bull. Sci. Math. 128 (2004), 7-22. 
[10] A. Buică; J. Llibre. Bifurcation of limit cycles from a four-dimensional center in control systems, Internacional Journal of Bif. and Chaos 15 (2005), 2653-2662.

[11] J. Chavarriga; M. Grau. Some open problems related to 16th Hilbert problem, Sci. Ser. A. Math. Sci. (N.S.) 9 (2003), 1-26.

[12] S. N. Chow; C. Li; D. Wang. Normal forms and bifurcation of planar vector fields. Cambridge University Press, 1994.

[13] C. Christopher; C. Li. Limit cycles of differential equations. Advanced course on limit cycles of differential equations. CRM Barcelona, Birkhäuser Verlag, 2007.

[14] L. Corry. Los 23 problemas de Hilbert y su trasfondo histórico. Bol. Asoc. Mat. Venez. 5 (1998), no. 2, 119-125.

[15] F. Dumortier; J. Llibre; J. C. Artés. Qualitative theory of planar differential systems. New York: Springer, 2006.

[16] J. Écalle. Introduction aux fonctions analysables et preuve constructive de la conjecture de Dulac. Paris: Actualités Math. Hermann, 1992.

[17] J. A. Ellison; A. W. Sáenz; H. S. Dumas. Improved Nth order averaging theory for periodic systems, J. Differential Equations 84 (1990), 383-403.

[18] R. D. Euzébio. O método do averaging via teoria do grau de Brouwer e aplicações. Dissertação (Mestrado em Matemática), IBILCE/Unesp, (2011).

[19] A. Ferragut; J. Llibre; M. A. Teixeira. Periodic orbits for a class of $C^{1}$ threedimensional systems, Rend. Circ. Mat. Palermo 56 (2007), 101-115.

[20] A. Gasull; H. Giacomini; J. Llibre. New criteria for the existence and non-existence of limit cycles in Liénard differential systems, Dyn. Sist. 24 (2009), 171-185.

[21] J. Guckenheimer; P. Holmes. Nonlinear oscillations, dynamical systems and bifurcations of vector fields. Applied Mathematical Sciences, v. 42. New York: SpringerVerlag, 1990. 
[22] Hartono; A. H. P. van der Burgh. Higher-order averaging: periodic solutions, linear systems and an application, Nonlinear Anal. 52 (2003), 1727-1744.

[23] J. K. Hale. Ordinary differential equations. New York: Wiley-Interscience, (1980).

[24] D. Hilbert. Mathematical problems. Reprinted from Bull. Amer. Math. Soc. 8 (1902), 437-479. Bull. Amer. Math. Soc. (N.S.) 37 (2000), 407-436.

[25] D. Hilbert. Mathematische probleme. In Nachr. Ges. Wiss., editor, Second Internat. Congress Math. Paris, 1900, Göttingen Math.-Phys. Kl. (1900), 253-297.

[26] I. D. Iliev. Perturbations of quadratic centers, Bull. Sci. Math. 122 (1998), 107-161.

[27] Y. Il'Yashenko. Finiteness theorem for limit cycles, Trans. of Math. Monogr. Am. Math. Soc. 94 (1991).

[28] A. Khovanskii. Real analytic manifolds with finiteness properties and complex abelian integrals, Funct. Anal. Appl. 18 (1984), 119-128.

[29] J. Li. Hilbert's 16th problem and bifurcations of planar polynomial vector fields, Internat. J. Bifur. Chaos Appl. Sci. Engrg. 13 (2003), 47-106.

[30] C. Li; W. Li; J. Llibre; Z. Zhang. Linear estimate for the number of zeros of Abelian integrals for quadratic isochronous centres, Nonlinearity 13, (2000), 1775-1800.

[31] C. Li; J. Llibre. The cyclicity of period annulus of a quadratic reversible LotkaVolterra system, Nonlinearity 22 (2009), 2971-2979.

[32] C. Li; Z. F. Zhang. A criterion for determining the monotonicity of the ratio of two abelian integrals, J. Differential Equations 124 (1996), 407-424.

[33] J. Llibre. Averaging theory and limit cycles for quadratic systems, Radovi Matematički 11 (2002), 215-228.

[34] J. Llibre; A. C. Mereu; M. A. Teixeira. Limit cycles of generalized polynomial Liénard differential equations, Math. Proc. Camb. Phil. Soc., 148 (2010), 363-383. 
[35] J. Llibre; R. D. S. Oliveira. Phase portraits of quadratic polynomial vector fields having a rational first integral of degree 3, Nonlinear Anal., Theory, Methods and Appl. 70 (2009), 3549-3560.

[36] J. Llibre; R. D. S. Oliveira. Correction to "Phase portraits of quadratic polynomial vector fields having a rational first integral of degree 3", Nonlinear Anal., Theory, Methods and Appl., 2009.

[37] J. Llibre; G Rodríguez. Configuration of limit cycles and planar polynomial vector fields, J. Differential Equations 198 (2004), 374-380.

[38] A. Mahdi. Integrable polynomial differential systems and their perturbations. Tese (Doutorado em Matemática) - UAB/Barcelona, Barcelona, (2008).

[39] A. C. O. Mereu. Perturbações de sistemas reversíveis. Tese (Doutorado em Matemática) - IMECC/Unicamp, Campinas, (2009).

[40] J. Murdock. Perturbations: theory and methods. SIAM, Philadelphia, 1999.

[41] J. A. Sanders; F. Verhulst; J. Murdock. Averaging methods in nonlinear dynamical systems. 2. ed. New York: Springer, 2007.

[42] J. B. da Silva Jr. O método averaging e aplicações. Dissertação (Mestrado em Matemática) - IBILCE/Unesp, São José do Rio Preto, (2009).

[43] J. Sotomayor. Lições de equações diferenciais ordinárias. Projeto Euclides. Rio de Janeiro: IMPA, 1979.

[44] A. N. Varchenko. An estimate of the number of zeros of an abelian integral depending on a parameter and limiting cycles, Funct. Anal. Appl. 18 (1984), 98-108.

[45] F. Verhulst. Nonlinear differential equations and dynamical systems. Berlin: SpringerVerlag, 1989.

[46] Y. Yan-Qian. Theory of limit cycles, Translations of Math. Monographs, v. 66, Amer. Math. Soc, Providence, 1986. 
[47] Z. Yulin; L. Weigu; L. Chengzhi; Z. Zhifen. Linear estimate of the number of zeros of abelian integrals for quadratic centers having almost all their orbits formed by cubics, Science in China (Series A). 45 (2002), 964-974.

[48] Z. Zhi-fen; D. Tong-ren; H. Wen-zao; D. Zhen-xi. Qualitative theory of differential equations. Translations of Mathematical Monographs, v. 101, Peking University Press, 1991. 